\title{
Model building from asymptotic safety with Higgs and flavor portals
}

\author{
Gudrun Hiller $\odot,{ }^{1}$ Clara Hormigos-Feliu $\odot,{ }^{1}$ Daniel F. Litim $\odot,{ }^{2}$ and Tom Steudtner ${ }^{2}$ \\ ${ }^{1}$ Fakultät Physik, TU Dortmund, Otto-Hahn-Straße 4, D-44221 Dortmund, Germany \\ ${ }^{2}$ Department of Physics and Astronomy, University of Sussex, Brighton BNI 9QH, United Kingdom
}

(Received 3 September 2020; accepted 16 October 2020; published 23 November 2020)

\begin{abstract}
We perform a comprehensive search for Standard Model extensions inspired by asymptotic safety. Our models feature a singlet matrix scalar field, three generations of vectorlike leptons, and direct links to the Higgs and flavor sectors via new Yukawa and portal couplings. A novel feature is that the enlarged scalar sector may spontaneously break lepton flavor universality. We provide a complete two-loop renormalization group analysis of the running gauge, Yukawa, and quartic couplings to find ultraviolet fixed points and the critical surface of parameters, i.e., the set of boundary conditions at the $\mathrm{TeV}$ scale for which models remain well-behaved and predictive up to the Planck scale without encountering Landau poles or instabilities. This includes templates for asymptotically safe Standard Model extensions that match the measured values of gauge couplings and the Higgs, top, and bottom masses. We further detail the phenomenology of our models covering production, decay, fermion mixing, anomalous magnetic moments, effects from scalar mixing and chiral enhancement, and constraints on model parameters from data. Signatures at proton-proton and lepton colliders, such as lepton flavor violation and displaced vertices, and the prospect for electric dipole moments or charged lepton-flavor-violating type processes are also indicated.
\end{abstract}

DOI: 10.1103/PhysRevD.102.095023

\section{INTRODUCTION AND BASIC SETUP}

\section{A. Motivation and background}

Ultraviolet (UV) fixed points play a central role for fundamental quantum field theories. They ensure that running couplings remain finite and well-defined even at highest energies such that cross sections or scattering amplitudes stay well-behaved. Important examples are given by asymptotic freedom of non-Abelian gauge interactions and the strong nuclear force, where the fixed point is noninteracting [1,2]. UV fixed points may also be interacting, a scenario known as asymptotic safety, and conjectured a while ago in both particle physics [3] and quantum gravity [4]. It implies that quantum scale invariance is achieved with some of the running couplings taking finite, instead of vanishing, values in the UV.

The field has taken up some speed recently due to the discovery that asymptotic safety is realized rigorously in models of particle physics [5-10]. Gauge fields are key for this to happen at weak coupling [6] alongside Yukawa and scalar interactions subject to certain constraints $[7,8]$. A

Published by the American Physical Society under the terms of the Creative Commons Attribution 4.0 International license. Further distribution of this work must maintain attribution to the author(s) and the published article's title, journal citation, and DOI. Funded by SCOAP ${ }^{3}$. typical asymptotically safe theory contains gauge fields with charged fermions and mesonlike scalars, with gauge groups being either unitary [5], orthogonal or symplectic [9], or of the product type [10] such as in the Standard Model (SM) [11]. Results also cover aspects of the quantum vacuum [12], higher order self-interactions [13], Abelian factors [14], proofs with supersymmetry [15], conformal windows of parameters [16], and radiative symmetry breaking [17]. In a related vein, the proposal that gauge-fermion theories with many flavors may also realize UV fixed points $[18,19]$ has received renewed interest as of late [5,20-26]. For further studies of ultraviolet stable fixed points in particle physics, see [27-38].

Asymptotically safe models of particle physics share many features of the SM such as non-Abelian gauge interactions, a flavorful fermion sector with Yukawa interactions, and a scalar sector. It is therefore natural to ask whether the SM can be extended into an asymptotically safe version of itself, and if so, what type of phenomenological signatures this would entail. First proposals $[11,14]$ have featured $N_{F}$ vectorlike fermions $\psi$ in general representations of the SM gauge groups and hypercharge, and a $N_{F} \times N_{F}$ mesonlike complex scalar singlet $S$. The new matter fields couple to the SM through the gauge interactions and a Higgs portal, while the beyond-the-StandardModel (BSM) Yukawa term

$$
\mathcal{L}_{y}=-y \operatorname{Tr}\left[\bar{\psi}_{L} S \psi_{R}+\text { H.c. }\right],
$$


TABLE I. Shown are the gauge representations $R_{3}, R_{2}$ and the hypercharges $Y$ of the new vectorlike leptons $\psi$ with respect to the SM gauge group $S U(3)_{C} \times S U(2)_{L} \times U(1)_{Y}$ for the six basic models A-F. Also indicated are the mixed Yukawa terms involving SM leptons, BSM leptons, and either the complex gauge singlet BSM scalar $S$ or the SM Higgs $H$ or its charged conjugate $\tilde{H}=i \sigma^{2} H^{*}$; Yukawa couplings with SM scalars (BSM scalars) are denoted by $\kappa\left(\kappa^{\prime}\right)$, respectively. The last column $Q_{F}=$ $T_{3}+Y$ denotes the electric charge of the $\psi$ states.

\begin{tabular}{cccc}
\hline \hline Model & $\left(R_{3}, R_{2}, Y\right)$ & Yukawa interactions in $\mathcal{L}_{\text {mix }}$ & $Q_{F}$ \\
\hline A & $(\mathbf{1}, \mathbf{1},-1)$ & $\kappa \bar{L} H \psi_{R}+\kappa^{\prime} \bar{E} S^{\dagger} \psi_{L}$ & -1 \\
B & $(\mathbf{1}, \mathbf{3},-1)$ & $\kappa \bar{L} H \psi_{R}$ & $-2,-1,0$ \\
C & $\left(\mathbf{1}, \mathbf{2},-\frac{1}{2}\right)$ & $\kappa \bar{E} H^{\dagger} \psi_{L}+\kappa^{\prime} \bar{L} S \psi_{R}$ & $-1,0$ \\
D & $\left(\mathbf{1}, \mathbf{2},-\frac{3}{2}\right)$ & $\kappa \bar{E} \tilde{H}^{\dagger} \psi_{L}$ & $-2,-1$ \\
E & $(\mathbf{1}, \mathbf{1}, 0)$ & $\kappa \bar{L} \tilde{H} \psi_{R}$ & 0 \\
F & $(\mathbf{1}, \mathbf{3}, 0)$ & $\kappa \bar{L} \tilde{H} \psi_{R}$ & $-1,0,+1$ \\
\hline \hline
\end{tabular}

inspired from exact models $[5,10,16]$, helps generate interacting UV fixed points for moderate or large $N_{F}$ $[11,14,34]$. Phenomenological signatures at colliders include long-lived particles, R-hadrons, and Drell-Yan production, with a scale of new physics potentially as low as a few $\mathrm{TeV}$ and "just around the corner" [11].

In this paper, we put forward a new set of models which, in addition to (1), are characterized by direct Yukawa interactions between SM and BSM matter fields [39,40]. We are particularly interested in the relevance of flavor portals for the high-energy behavior of SM extensions, in the new phenomena that arise from them, and in their interplay with the Higgs portal. We focus on those settings where the new fermions $\psi$ are vectorlike and colorless. Moreover, to connect to SM flavor, we use $N_{F}=3$, that is, three generations of SM and BSM matter. These choices restrict the mixed Yukawa interactions to the leptons and leave us with a small number of viable $S U(2)$ gauge representations and hypercharges for the new fermions $\psi$ (see Table I), whose features and phenomenology are studied in depth.

\section{B. Setup for models with flavor portals}

In the remainder of the Introduction, we detail the basic setup and rationale for our choice of models and flavor symmetries. The renormalizable Lagrangians of the six basic models are given by

$$
\begin{aligned}
\mathcal{L} & =\mathcal{L}_{\mathrm{SM}}+\mathcal{L}_{\mathrm{BSM}}, \\
\mathcal{L}_{\mathrm{BSM}} & =\operatorname{Tr} \bar{\psi} i \not D \psi+\operatorname{Tr}\left[\left(\partial_{\mu} S\right)^{\dagger}\left(\partial^{\mu} S\right)\right]+\mathcal{L}_{\mathrm{s}}+\mathcal{L}_{\mathrm{Y}},
\end{aligned}
$$

where $\mathcal{L}_{\mathrm{SM}}$ denotes the SM Lagrangian and traces are over flavor indices. Throughout, we often suppress the flavor index of leptons and $\psi$ 's, as well as of the scalar matrix $S$. The term $\mathcal{L}_{\mathrm{s}}$ contains the BSM scalar self-interactions and the Higgs portal coupling, and

$$
\mathcal{L}_{\mathrm{Y}}=\mathcal{L}_{y}+\mathcal{L}_{\text {mix }}
$$

contains the Yukawa interactions amongst the new matter fields (1) and those between BSM and SM matter $\mathcal{L}_{\text {mix }}$. The latter are specified in Table I for the six basic models to which we refer to as models A-F. The SM fermionic content is denoted as $L, E$ for the lepton $S U(2)_{L}$ doublet and singlet, respectively, while $H$ denotes the SM Higgs doublet.

We can immediately state some of the new phenomenological features due to the flavor portal, with specifics depending on mass hierarchies and the flavor structure of Yukawa couplings mixing SM and BSM fields:

(i) The BSM sector decays to SM particles.

(ii) The BSM sector can be tree-level produced at colliders in pairs or singly.

(iii) An opportunity to address flavor data shifted a few standard deviations away from SM predictions. For example, the anomalous magnetic moments of the muon and the electron can be explained simultaneously with the mixed Yukawas in models A and C, without the necessity to manifestly break lepton flavor universality [40].

(iv) Flavor off-diagonal scalars $S_{i j}, i \neq j$ couple to different generations of fermions. Leptons and new fermions mix after electroweak symmetry breaking and lead to charged lepton flavor violation (LFV)-like signals from off-diagonal scalar decays $S_{i j} \rightarrow \ell_{i}^{ \pm} \ell_{j}^{\mp}(\ell=e, \mu, \tau)$.

Below, we give a general discussion of all models regarding SM tests with leptons, including prospects for magnetic and electric dipole moments.

Another important part of our study is to ensure that models remain finite and well-defined up to the Planck scale or beyond, for which we perform a complete two-loop renormalization group (RG) study of all models. To keep the technical complexity at bay, we make a few pragmatic and symmetry-based assumptions for the flavor structure of the new Yukawa interactions.

To that end, we consider the kinetic part of the Lagrangian (2). Its large flavor symmetry $\mathcal{G}_{F}$ can be decomposed as

$$
\mathcal{G}_{F}=U(3)_{q}^{3} \otimes U(3)_{\ell}^{2} \otimes U(3)_{\psi}^{2} \otimes U(3)_{S}^{2},
$$

with

$$
\begin{aligned}
U(3)_{q}^{3} & =U(3)_{Q} \otimes U(3)_{U} \otimes U(3)_{D}, \\
U(3)_{\ell}^{2} & =U(3)_{L} \otimes U(3)_{E}, \\
U(3)_{\psi}^{2} & =U(3)_{\psi_{L}} \otimes U(3)_{\psi_{R}}, \\
U(3)_{S}^{2} & =U(3)_{S_{L}} \otimes U(3)_{S_{R}}
\end{aligned}
$$

corresponding to the quarks, leptons, BSM fermions, and BSM scalars, respectively. The Yukawas, in general, do not 
respect the global symmetry (4). For instance, the SM part $U(3)_{q}^{3} \otimes U(3)_{\ell}^{2}$ is broken down to baryon number, lepton number, and hypercharge by the SM Yukawas of quarks and leptons. Assuming that some subgroup of $\mathcal{G}_{F}$ is left intact then dictates the flavor structure of the Yukawas. For example, without any assumptions on flavor the BSM Yukawa interactions would read

$$
y_{i j k \ell} \bar{\psi}_{L i} S_{j k} \psi_{R \ell}
$$

with $3^{4}$ independent Yukawa couplings $y_{i j k \ell}$. However, identifying $U(3)_{S}^{2}$ with $U(3)_{\psi_{L}} \otimes U(3)_{\psi_{R}}$, the symmetrypreserving Yukawa interaction is given by (1) with a universal coupling $y$ instead [5,11].

Similarly, the mixed fermion couplings with the singlet scalars $\left(\kappa^{\prime}\right)$ in Table I also carry four flavor indices in general. To simplify the flavor structure along the lines of (6) versus (1) we identify $U(3)_{E}$ with $U(3)_{\psi_{R}}$ (model A) or $U(3)_{L}$ with $U(3)_{\psi_{L}}$ (model C). As a result, the interactions are driven by a single Yukawa coupling instead of a tensor and read

$$
\begin{aligned}
& \kappa^{\prime} \operatorname{Tr}\left[\bar{E} S^{\dagger} \psi_{L}+\text { H.c. }\right](\text { model A }), \\
& \kappa^{\prime} \operatorname{Tr}\left[\bar{L} S \psi_{R}+\text { H.c. }\right](\text { model C }) .
\end{aligned}
$$

Finally, all models in Table I contain the mixed HiggsYukawa matrix $(\kappa) .{ }^{1}$ In models A, B, E, and F we identify $U(3)_{L}$ with $U(3)_{\psi_{R}}$ and in models $\mathrm{C}$ and $\mathrm{D}$ we identify $U(3)_{E}$ with $U(3)_{\psi_{L}}$, which results in a diagonal and universal Yukawa coupling

$$
\kappa_{i j}=\kappa \delta_{i j} \quad(\text { models A-F }) .
$$

Incidentally, the flavor symmetry for models A and C entails that $\kappa$ is proportional to the SM lepton Yukawa coupling in $Y_{\ell} \bar{L} H E+$ H.c. implying that the latter is flavor-diagonal $Y_{\ell} \sim 1$. However, the SM lepton Yukawa couplings are irrelevant and will be neglected, unless stated otherwise. Alternatively, we could have fixed the flavor symmetry by identifying $U(3)_{E} \sim U(3)_{\psi_{R}}$ (models $\mathrm{B}$, E, and $\mathrm{F}$ ), or $U(3)_{L} \sim U(3)_{\psi_{L}}$ (model D), to find hierarchical Yukawas

$$
\kappa \sim Y_{\ell} \quad(\text { models B }, \mathrm{D}, \mathrm{E}, \mathrm{F})
$$

instead of (8). Again, we do not pursue this path any further as the lepton Yukawas are neglected in the RG study, and adopt (8) for all models. In all scenarios, BSM fermion mass terms $\bar{\psi}_{L} M_{F} \psi_{R}+$ H.c. break the respective remaining symmetries unless $U(3)_{\psi_{L}} \sim U(3)_{\psi_{R}}$, which gives universal and diagonal $M_{F}$ in all models.

\footnotetext{
${ }^{1}$ Notice that we keep the SM Higgs unflavored.
}

The symmetry language provides guidance for minimal benchmarks with reduced numbers of parameters (entries in Yukawa tensors). This makes the study manageable and structures the RG equations. If the origin of flavor would, in fact, be symmetries, there is a fundamental reduction in complexity, and new physics patterns observed can provide feedback on flavor [41]. In the following we use the Yukawa interactions (3) together with (7) and (8). Unless stated otherwise, we also assume that all BSM couplings are realvalued.

\section{Outline}

The remaining parts of the paper are organized as follows. In Sec. II we recall the tools for asymptotic safety of weakly coupled gauge theories with matter covering interacting fixed points, scaling exponents, vacuum stability, the critical surface of parameters, and the matching to the Standard Model. In Sec. III, a detailed "top-down" search of fixed points, RG flows, and matching conditions is provided for all models to the leading nontrivial orders in perturbation theory.

In Sec. IV, the impact of the scalar sector and the interplay between the Higgs and flavor portals are investigated. RG trajectories from the $\mathrm{TeV}$ to the Planck scale are studied in a "bottom-up" search at the complete two-loop accuracy for the top, bottom, and new Yukawas, and all gauge and quartic couplings. The BSM critical surface of parameters, i.e., the parameter regions of BSM couplings at the $\mathrm{TeV}$ scale which lead to well-defined (stable vacua, no Landau poles) models up to the Planck scale or beyond, is identified.

In Sec. V, we concentrate on the phenomenology of our models covering production, decay, fermion mixing, and constraints on model parameters from data. Effects from scalar mixing and chiral enhancement, the prospects for anomalous magnetic moments, electric dipole moments (EDMs) or LFV-type processes, and signatures at $p p$ and lepton colliders such as lepton flavor violation and displaced vertices, are also worked out. We summarize in Sec. VI. Some auxiliary information and formulas are relegated into Appendixes (Appendixes A-E).

\section{TOOLS FOR ASYMPTOTIC SAFETY}

In this section, we recall the principles and basic tools for asymptotic safety and adopt them to the models at hand. Asymptotic safety requires that the couplings of a theory approach renormalization group fixed points in the highenergy limit. In the language of the renormalization group, fixed points correspond to zeros of $\beta$-functions

$$
\left.\left.\beta_{a}(\alpha)\right|_{\alpha=\alpha^{*}} \equiv \frac{d \alpha_{a}}{d \ln \mu}\right|_{*}=0
$$

for all couplings $\alpha_{a}$, with $\alpha_{a}^{*}$ denoting the fixed point coordinates. Fixed points can be fully interacting with all 
couplings nonzero or partially interacting whereby some couplings become free in the UV.

Thus, the first step is to compute the $\beta$-functions and determine whether fixed points exist. This will be achieved using [42-48]. Then, one must study if the fixed points can be reached from the IR and, finally, if the trajectories can be matched to the SM.

\section{A. Renormalization group}

We are interested in free or interacting ultraviolet (UV) fixed points in extensions of the SM. The three gauge couplings corresponding to the $U(1)_{Y}, S U(2)_{L}$, and $S U(3)_{C}$ gauge sectors are introduced as

$$
\alpha_{1}=\frac{g_{1}^{2}}{(4 \pi)^{2}}, \quad \alpha_{2}=\frac{g_{2}^{2}}{(4 \pi)^{2}}, \quad \alpha_{3}=\frac{g_{3}^{2}}{(4 \pi)^{2}},
$$

respectively. In our setup, the BSM fermions do not introduce new $S U(3)$ gauge charges meaning that the strong coupling continues to have an asymptotically free UV fixed point. One may therefore neglect $\alpha_{3}$ for the fixed point search: we actually do so in the lowest order analysis in Sec. III, but treat $\alpha_{3}$ at the same order as the electroweak couplings in the SM-RG and in the higher order analysis in Sec. IV. On the other hand, the BSM fermions carry hypercharge and/or weak charges; see Table I. Hence, the weak (hypercharge) coupling is infrared free in some (in all) models and requires an interacting UV fixed point to help cure potential Landau poles and the triviality problem.

At weak coupling, interacting UV fixed points arise in exactly two manners [6,7]. An infrared free gauge theory can either directly develop an UV fixed point with the help of Yukawa interactions or may become asymptotically free owing to a gauge-Yukawa (GY) fixed point involving other gauge couplings $[7,15]$. Either way, Yukawa interactions are key for a well-behaved UV limit. The Yukawa couplings which may take this role in our models are those given in (3) and Table I. We write them as

$$
\alpha_{y}=\frac{y^{2}}{(4 \pi)^{2}}, \quad \alpha_{\kappa}=\frac{\kappa^{2}}{(4 \pi)^{2}}, \quad \alpha_{\kappa^{\prime}}=\frac{\kappa^{\prime 2}}{(4 \pi)^{2}} .
$$

Let us now turn to the renormalization group equations for weakly coupled semisimple gauge theory with $n_{G}$ gauge couplings $\alpha_{i}$ and $n_{Y}$ Yukawa couplings $\alpha_{n}$ amongst matter fields [7]. Our models have three gauge couplings $(i=1,2,3)$ and up to three BSM Yukawa couplings $\left(n=y, \kappa, \kappa^{\prime}\right)$, plus SM Yukawas and quartics.

Two remarks on notation: unless indicated otherwise we use the letters $i, j$ as indices for gauge couplings, the letters $n, m$ as indices for Yukawa couplings, and the letters $a, b, c$ as indices for any of the gauge, Yukawa, or scalar couplings. Following $[5,16]$, we also introduce the notation $\mathrm{klm}$ to denote a perturbative approximation of beta functions which retains $\mathrm{k}$ loop orders in the gauge beta function, 1 loops in the Yukawa, and $\mathrm{m}$ loops in the scalar beta functions.

With these conventions in mind, the gauge beta functions are given by

$$
\begin{aligned}
\beta_{i} & \equiv \frac{d \alpha_{i}}{d \ln \mu} \\
& =-\alpha_{i}^{2}\left(B_{i}-\sum_{j=\text { gauge }} C_{i j} \alpha_{j}+\sum_{n=\text { Yukawa }} D_{\text {in }} \alpha_{n}\right)+\mathcal{O}\left(\alpha^{3}\right)
\end{aligned}
$$

at the leading nontrivial order in perturbation theory which is the 210 approximation. The one-loop coefficients $B_{i}$ and the diagonal two-loop gauge coefficients $C_{i i}$ (no sum) may take either sign depending on the matter content, though for $B_{i}<0$ the latter is always positive. The two-loop Yukawa coefficients $D_{\text {in }}$ and the off-diagonal elements $C_{i j}(i \neq j)$ are always positive for any quantum field theory. In these conventions, the gauge coupling $\alpha_{i}$ is asymptotically free if $B_{i}>0$. Similarly, the Yukawa beta functions take the form

$\beta_{n} \equiv \frac{d \alpha_{n}}{d \ln \mu}=\alpha_{n}\left(\sum_{m=\text { Yukawa }} E_{n m} \alpha_{m}-\sum_{i=\text { gauge }} F_{n i} \alpha_{i}\right)+\mathcal{O}\left(\alpha^{2}\right)$.

Any of the loop coefficients $E$ and $F$ are positive in any quantum field theory. The loop coefficients in (13) and (14) corresponding to our models can be found in Appendix A.

\section{B. Ultraviolet Fixed Points}

Next, we turn to renormalization group fixed points. Yukawa couplings at a fixed point are either free or interacting, and ultraviolet fixed points require that some (or all) Yukawa couplings are nonzero. The vanishing of (14) implies that the nonzero Yukawa couplings are related to the gauge couplings as

$$
\alpha_{n}=\left(E^{-1}\right)_{n m} F_{m j} \alpha_{j} .
$$

We refer to these relations as the Yukawa nullclines. Notice that the matrix $E$ is inverted over the set of nonvanishing Yukawa couplings, and the matrix multiplication in (15) excludes the vanishing Yukawa couplings (if any). In theories with $n_{Y}$ Yukawa couplings this procedure can lead to as many as $2^{n_{Y}}-1$ different nullclines. Fixed points for the gauge coupling are found by inserting the nullcline (15) into (13), leading to

$$
\left.\beta_{i}\right|_{\beta_{n}=0}=-\alpha_{i}^{2}\left(B_{i}-C_{i j}^{\prime} \alpha_{j}\right) .
$$

Hence, every Yukawa nullcline generates shifted two-loop coefficients $C^{\prime}$ given by 


$$
C_{i j}^{\prime}=C_{i j}-D_{\text {in }}\left(E^{-1}\right)_{n m} F_{m j}
$$

in terms of the perturbative loop coefficients. In particular, the nonzero fixed points for the gauge couplings follow from (16) and (17) as

$$
\alpha_{i}^{*}=\left(C^{\prime-1}\right)_{i j} B_{j},
$$

where the sum over $j$ only includes the nonvanishing gauge couplings. The Yukawa fixed point follows from inserting (18) into the corresponding nullcline (15). Overall, we may find up to $\left(2^{n_{G}}-1\right)\left(2^{n_{Y}}-1\right)$ different gauge-Yukawa fixed points. Also notice that the physicality condition $\alpha_{i \ldots}^{*}, \alpha_{n}^{*} \ldots \geq 0$ is not guaranteed automatically and must still be imposed. Viable gauge-Yukawa fixed points genuinely exist for asymptotically free gauge sectors. Most importantly, thanks to the Yukawa-induced shift in (17), physical solutions (18) may even exist for infrared free gauge sectors where $B_{i}<0$. This is the primary mechanism to stabilize infrared free gauge sectors in the UV.

Gauge-Yukawa fixed points may also indirectly stabilize an otherwise infrared free gauge sector $[7,11,14,15]$, because the one-loop coefficient of a gauge theory can be modified in the presence of an interacting fixed point. Conditions for this to happen for an infrared free gauge coupling $\alpha_{i}$ can now be read off from (13),

$$
B_{i}^{\text {eff }}=B_{i}-C_{i j} \alpha_{j}^{*}+D_{\text {in }} \alpha_{n}^{*}
$$

The sums run over the nonzero gauge and Yukawa couplings $\left\{\alpha_{j \ldots}^{*}, \alpha_{n \ldots}^{*}\right\}$, and we recall that $\alpha_{i}^{*}=0$. Provided that the effective one-loop coefficient becomes positive, $B_{i}^{\text {eff }}>0>B_{i}$, the infrared free gauge coupling becomes free in the ultraviolet. This is the secondary mechanism to stabilize infrared free gauge sectors in the UV. We stress that Yukawa couplings are mandatory for this as they are the only couplings contributing positively to (19). Below, we will see that both mechanisms are operative in our models.

If all Yukawa couplings vanish, the gauge sector (13) may still achieve free or interacting fixed points. The interacting ones are given by

$$
\alpha_{i}^{*}=\left(C^{-1}\right)_{i j} B_{j},
$$

where the sum runs over the nonzero gauge couplings. These are the well-known Banks-Zaks (BZ) fixed points [49,50], which are always infrared and can only be physical $\left(\alpha_{i}^{*}>0\right)$ for asymptotically free gauge couplings. In theories with $n_{G}$ asymptotically free gauge couplings, we may find up to $2^{n_{G}}-1$ of them. Although BanksZaks fixed points play no role for the UV completion of theories, they may still be present and influence the RG evolution of couplings on UV-IR connecting trajectories.

\section{Scalar potential and Higgs portal}

Here we briefly discuss the scalar sector and its ground states. As the BSM scalar carries flavor and couples to the SM fermions, its vacuum expectation values (VEVs) have implications for the flavor structure of the model.

The minimal potential involving the SM and BSM scalars $H$ and $S$ included in (2) and compatible with the symmetries (4) has the form

$$
\begin{aligned}
V(H, S)= & -\mu^{2} H^{\dagger} H-\mu_{s}^{2} \operatorname{Tr}\left[S^{\dagger} S\right]-\mu_{\operatorname{det}}\left(\operatorname{det} S+\operatorname{det} S^{\dagger}\right) \\
& +\lambda\left(H^{\dagger} H\right)^{2}+\delta H^{\dagger} H \operatorname{Tr}\left[S^{\dagger} S\right] \\
& +u \operatorname{Tr}\left[S^{\dagger} S S^{\dagger} S\right]+v\left(\operatorname{Tr}\left[S^{\dagger} S\right]\right)^{2}
\end{aligned}
$$

for all models. It consists of the Higgs self-coupling $\lambda$ and mass parameter $\mu$, the BSM scalar quartics $u, v$, as well as the BSM mass parameters $\mu_{s}$ and the trilinear coupling $\mu_{\mathrm{det}}$, and a portal coupling $\delta$ which mixes SM and BSM scalars. Viable UV fixed points for our models require that the Higgs self-coupling, the portal coupling, and the selfcouplings of the BSM scalar fields take fixed points by themselves, compatible with vacuum stability. Interestingly though, the quartics do not couple back into the gaugeYukawa system at the leading order. Rather, fixed points in the SM and BSM scalar sectors are fueled by the gaugeYukawa fixed points, and backcoupling occurs starting at the two-loop level in the Yukawa sector and at the threeloop level for the Higgs (four-loop for the BSM scalars) in the gauge sectors.

The classical moduli space for (21) and conditions for the asymptotic stability of the vacuum are found following [12,51]. Depending on the sign of $u$, we find two settings $V^{ \pm}$with stability conditions

$$
\begin{aligned}
& V^{+}: \begin{cases}\lambda>0, & u>0, \\
\delta>-2 \sqrt{\lambda(u / 3+v)}, & u+3 v>0,\end{cases} \\
& V^{-}: \begin{cases}\lambda>0, & u<0, \\
\delta>-2 \sqrt{\lambda(u+v)} . & u+v>0,\end{cases}
\end{aligned}
$$

Both settings allow for the Higgs to break electroweak symmetry. For $V^{+}$, the BSM scalar VEV is flavor-diagonal and upholds some notion of flavor universality in interactions with the SM. On the other hand, $V^{-}$has a VEV only in one diagonal component of $S$. In the context of our models, this corresponds to a VEV pointing in the direction of one lepton flavor. We learn that the Lagrangian (2) offers the possibility to violate lepton flavor universality spontaneously, an interesting feature also in the context of today's flavor anomalies, e.g., [52]. Note if both scalars $S$ and $H$ acquire a VEV, the portal coupling $\delta$ induces mixing between the scalars $H$ and $S$. Details can be seen in Appendix D. In the following we investigate the availability of fixed points, vacuum stability, and phenomenological 
signatures at various orders in perturbation theory up to the 222 approximation using the methodology of [42-48].

\section{Scaling exponents and UV critical surface}

The renormalization group flow in the vicinity of fixed point provides information on whether the fixed point can be approached in the UV or IR. Denoting by $\alpha_{a}$ any of the gauge, Yukawa, or scalar couplings, and expanding the $\beta$-functions around a fixed point $\alpha_{a}^{*}$ up to second order in $\delta_{a}=\alpha_{a}-\alpha_{a}^{*}$, we find

$$
\beta_{a}=M_{a b} \delta_{b}+P_{a b c} \delta_{b} \delta_{c}+\mathcal{O}\left(\delta^{3}\right),
$$

where $M_{a b}=\partial \beta_{a} /\left.\partial \alpha_{b}\right|_{*}$ is the stability matrix and $P_{a b c}=\frac{1}{2} \partial^{2} \beta_{a} /\left.\partial \alpha_{b} \partial \alpha_{c}\right|_{*}$. After diagonalizing $M$ the running of couplings at first order may be written as

$$
\alpha_{a}(\mu)=\alpha_{a}^{*}+\sum_{b} V_{a}{ }^{b} c_{b}(\mu / \Lambda)^{\vartheta_{b}},
$$

where $\mu$ is the RG scale and $\Lambda$ a UV reference scale, while the UV scaling exponents $\vartheta_{b}$ arise as the eigenvalues of the stability matrix $M$ with $V^{b}$ the corresponding eigenvectors and $c_{b}$ free parameters. An eigenvector is relevant, marginal, or irrelevant if the corresponding eigenvalue $\vartheta$ is negative, zero, or positive. For all relevant and marginally relevant couplings, the parameters $c_{b}$ are fundamentally free and constitute the "UV critical surface" of the theory. Its dimension should be finite to ensure predictivity. For all irrelevant couplings, we must set $c_{b} \equiv 0$ or else the UV fixed point cannot be reached in the limit $\mu \rightarrow \infty$. UV fixed points require at least one relevant or marginally relevant eigendirection.

If a fixed point is partially interacting, that is, some but not all couplings are nonzero, the relevancy of the vanishing couplings can be established as follows. If a gauge coupling $\alpha_{i}$ vanishes at a fixed point, it follows from (13) being at least quadratic in $\alpha_{i}$ that the coupling is marginal. Going to second order in perturbations (23) reveals that $P_{i i i}=-B_{i}^{\text {eff }}$. As expected, the sign of (19) determines whether the coupling is marginally relevant $\left(B_{i}^{\text {eff }}>0\right)$ or marginally irrelevant. If a Yukawa coupling $\alpha_{n}$ vanishes at a GY fixed point with coordinates $\left\{\alpha_{i \ldots}^{*}, \alpha_{m \ldots}^{*}\right\}$, it follows from (14) that the corresponding scaling exponent is given by

$$
\vartheta_{n}=E_{n m} \alpha_{m}^{*}-F_{n i} \alpha_{i}^{*}
$$

As this is a difference between two positive numbers, its overall sign is not determined by the existence of the fixed point and the coupling could come out as relevant, marginal, or irrelevant. For BZ fixed points (all $\alpha_{m}^{*}=0$ ), however, the eigenvalue is always negative and the Yukawas are relevant.

\section{E. Matching and BSM critical surface}

Here we consider how an asymptotically safe UV fixed point must be connected to the SM. At low energies, any extension of the SM must connect to the measured values of SM couplings. For simplicity, and without loss of generality, we assume that all BSM matter fields have identical masses $M_{F}$. Moreover, the decoupling of heavy modes is approximated by considering the BSM fields either as massless (for $\mu>M_{F}$ ) and as infinitely massive (for $\mu<M_{F}$ ). Both of these technical assumptions can be lifted to account for a range of BSM matter field masses, and for a smooth decoupling of heavy modes, without altering the main pattern. In this setting, the fluctuations of BSM fields are absent as soon as $\mu<M_{F}$, meaning that the running of all SM couplings $\alpha_{\mathrm{SM}}(\mu)$ must be identical to the known SM running for all $\mu \leq M_{F}$. Therefore, we refer to

$$
\mu=M_{F}
$$

as the matching scale. On the other hand, the values of the BSM couplings $\alpha_{\mathrm{BSM}}(\mu)$ at the matching scale (26) are not predicted by the SM and must be viewed as free parameters. Schematically, we denote this set of free parameters as

$$
S_{\text {free }}=\left\{\alpha_{\mathrm{BSM}}\right\} .
$$

Any BSM renormalization group trajectory is uniquely characterized by the matching scale (26), the (known) values of SM couplings at the matching scale, and the initial values of BSM couplings (27). The latter are, in our models, the values of the three BSM scalar couplings plus the two (or three) BSM Yukawa couplings at the scale $M_{F}$,

$$
\alpha_{\mathrm{BSM}}=\left(\alpha_{y}, \alpha_{\kappa}, \alpha_{\kappa^{\prime}}, \alpha_{\delta}, \alpha_{u}, \alpha_{v}\right),
$$

and the parameter space (27) is hence five (or six) dimensional, depending on the model.

Depending on the BSM initial values (27), renormalization group trajectories may display a variety of different patterns. These include a power-law approach toward an interacting fixed point, a crossover through a succession of fixed points such as in asymptotic safety proper, or a logarithmically slow decay toward the free fixed point such as in asymptotic freedom. Either of these behaviors or, in fact, any combination thereof, corresponds to a viable highenergy limit in the sense of Wilson's path integral definition of quantum field theory. In turn, couplings may also run into unphysical regimes where the quantum vacuum becomes meta- or unstable, or where couplings become nonperturbatively large and RG trajectories terminate due to Landau pole singularities.

From a bottom-up model building perspective, the set of parameter values $S_{\mathrm{BSM}}$ for which the BSM trajectories remain finite and well-behaved-at least up to the Planck scale - is of particular interest. First and foremost, this set 
TABLE II. Fixed points of model $\mathrm{A}$ in the 210 approximation. $\mathrm{FP}_{1,2,3}$ are IR or crossover fixed points, $\mathrm{FP}_{4}$ is a line of fixed points, and $\mathrm{FP}_{5,6,7,8}$ are UV fixed point candidates. Also shown are the numbers of relevant ("Rel.") and irrelevant ("Irrel.") eigendirections, and whether the fixed point is of the BZ or GY type, with indices specifying the nontrivial couplings. Free couplings with power-law running are marked with a superscript \pm if they are irrelevant/relevant, and an additional parenthesis $( \pm)$ indicates that the flow is logarithmic; see Figs. 1-3 for the phase diagram and sample trajectories.

\begin{tabular}{|c|c|c|c|c|c|c|c|c|c|c|}
\hline Model A & $\alpha_{1}^{*}$ & $\alpha_{2}^{*}$ & $\alpha_{\kappa}^{*}$ & $\alpha_{\kappa^{\prime}}^{*}$ & $\alpha_{y}^{*}$ & Rel. & Irrel. & Info & Fig. 1 & Matching \\
\hline $\mathrm{FP}_{1}$ & $0^{(+)}$ & $0^{(-)}$ & $0^{(+)}$ & $0^{(+)}$ & $0^{(+)}$ & 1 & 4 & Saddle & & \\
\hline $\mathrm{FP}_{2}$ & $0^{(+)}$ & 0.543 & $0^{-}$ & $0^{(+)}$ & $0^{(+)}$ & 1 & 4 & $\mathrm{BZ}_{2}$ & & \\
\hline $\mathrm{FP}_{3}$ & $0^{(+)}$ & 0.623 & 0.311 & $0^{(+)}$ & $0^{+}$ & 0 & 5 & $\mathrm{GY}_{2 \kappa}$ & & \\
\hline $\mathrm{FP}_{4}$ & 2.746 & $0^{(+)}$ & $0^{-}$ & $4.120-\alpha_{y}^{*}$ & $\alpha_{y}^{*}$ & 2 & 2 & Line & & \\
\hline $\mathrm{FP}_{5}$ & 1.063 & $0^{(-)}$ & 0.886 & 1.594 & $0^{+}$ & 2 & 3 & $\mathrm{GY}_{1 \kappa \kappa^{\prime}}$ & $A_{1} \checkmark$ & (Fig. 3) \\
\hline $\mathrm{FP}_{6}$ & 1.105 & 0.569 & 1.205 & 1.657 & $0^{+}$ & 1 & 4 & $\mathrm{GY}_{12 \kappa \kappa^{\prime}}$ & $A_{2} \times$ & (Fig. 2) \\
\hline $\mathrm{FP}_{7}$ & 2.151 & $0^{(-)}$ & 0.782 & $0^{-}$ & 3.032 & 3 & 2 & $\mathrm{GY}_{1 y \kappa}$ & $A_{3} \checkmark$ & \\
\hline $\mathrm{FP}_{8}$ & 2.267 & 0.200 & 0.933 & $0^{-}$ & 3.165 & 2 & 3 & $\mathrm{GY}_{12 y \kappa}$ & $A_{4} X$ & \\
\hline
\end{tabular}

includes initial values for all trajectories that terminate at interacting UV fixed points, should they exist. In general, however, it can often be larger, simply because it may also include trajectories that remain finite and well-defined up to the Planck scale, but would otherwise not reach an interacting UV fixed point proper in the trans-Planckian regime. This feature can be referred to as Planck safety [40], as opposed to as well as extending the notion of asymptotic safety. The set of viable BSM parameters $S_{\mathrm{BSM}}$ is a subset of (27), and often of a lower dimensionality. The reason for this is that interacting UV fixed points have relevant and irrelevant eigenoperators. All interactions that are irrelevant in the UV impose constraints on the viable values of BSM couplings at the matching scale (27). Therefore, we refer to the set of viable initial values $S_{\mathrm{BSM}}$ as the "BSM critical surface." We obtain BSM critical surfaces for models A-F in Sec. IV E.

\section{BENCHMARK MODELS AND FIXED POINTS}

In this section we further specify our benchmark models and investigate their RG flows to the leading nontrivial order in perturbation theory. We focus on the gauge and the Yukawa couplings whose beta functions are given by (13) and (14) with loop coefficients for all models stated in Sec. A. Our goal is to gain a first understanding of models and fixed points, and the availability of matchings to the SM. We postpone the study of quartic scalar couplings and higher order loop corrections to Sec. IV.

The leading order approximation-known as the 210 approximation-retains two-loop orders in the gauge and one-loop in the Yukawa couplings. Scalar couplings are neglected. Besides the free Gaussian fixed point, we may find interacting Banks-Zaks or gauge-Yukawa fixed points, though only the latter will qualify as UV fixed points. Already at this order in the approximation, there can be up to a maximum of $\left(2^{n_{G}}-1\right)$ different Banks-Zaks and a maximum of $\left(2^{n_{G}}-1\right) \times\left(2^{n_{Y}}-1\right)$ different $G Y$ fixed points $[7,10]$. Here $n_{G}$ denotes the number of SM gauge groups under which the BSM fermions are charged $\left(n_{G}=2,1\right.$, or 0 for our models), and $n_{Y}$ the number of BSM Yukawa couplings $\left(n_{Y}=2\right.$ or 3 for all models). For this reason, for Banks-Zaks fixed points in semisimple gauge theories we specify the nonzero gauge couplings as an index (e.g., BZ $Z_{2}$ ). Similarly, for gauge-Yukawa fixed points, we also indicate the nonvanishing Yukawa couplings (e.g., $\mathrm{GY}_{1 \kappa}$ ).

Findings of this section are summarized in Sec. III G.

\section{A. Model A (singlets, $Y=-1)$}

Model A consists of the SM, amended by complex singlet BSM scalars $S$ and $N_{F}=3$ vectorlike BSM fermions $\psi$ in the representation $(\mathbf{1}, \mathbf{1},-1)$, which is identical to the one of the singlet leptons $E$ present in the SM, with Lagrangian (2). The Yukawa sector (3) contains three BSM couplings,

$$
-\mathcal{L}_{\mathrm{Y}}^{\mathrm{A}}=\kappa \bar{L} H \psi_{R}+\kappa^{\prime} \bar{E} S^{\dagger} \psi_{L}+y \bar{\psi}_{L} S \psi_{R}+\text { H.c. }
$$

Fixed points for model A are summarized in Table II and denoted as $\mathrm{FP}_{1}-\mathrm{FP}_{8}$. Table II also shows the number of relevant and irrelevant eigendirections. Free couplings are marked with a superscript + if they are irrelevant or with a if they are relevant, with power-law running. An additional parenthesis, that is, (+) or (-) for irrelevant or relevant, respectively, indicates that the flow along its eigendirection is logarithmically slow instead. It is also shown whether a fixed point is of the BZ or GY type, in which case an index is added to specify the nontrivial couplings.

The Gaussian fixed point $\left(\mathrm{FP}_{1}\right)$ is a saddle owing to $B_{1}<$ $0<B_{2}$ and takes the role of a crossover fixed point. $\mathrm{FP}_{2}$ is an infrared Banks-Zaks fixed point $\left(\mathrm{BZ}_{2}\right)$ where the Yukawa coupling $\alpha_{\kappa}$ is the sole relevant coupling because the fermions $\psi, E$ do not carry weak isospin. $\mathrm{FP}_{3}$ is an infrared gauge-Yukawa fixed point $\left(\mathrm{GY}_{2 \kappa}\right)$ which acts as an infrared sink because it is fully attractive in all canonically 

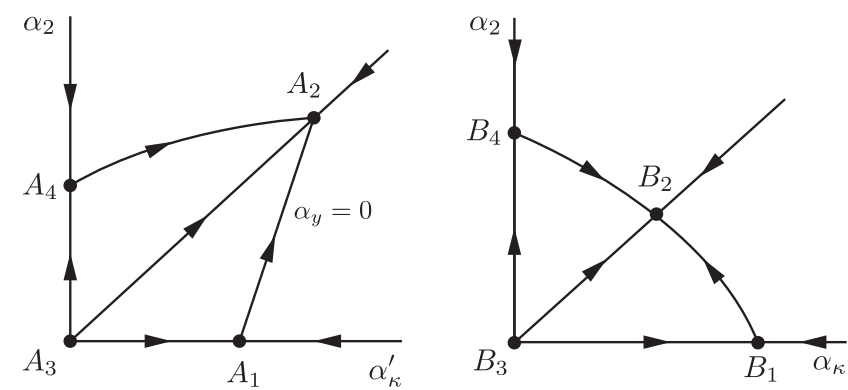

FIG. 1. Schematic phase diagram and various UV fixed points of models A, B, and D in the 210 approximation. Arrows indicate the flow from the UV to the IR. The fixed points of model A (Table II) are projected onto the $\left(\alpha_{2}, \alpha_{\kappa^{\prime}}\right)$ plane (left panel) with $A_{2}$ denoting the least and $A_{3}$ the most ultraviolet attractive fixed point. The fixed points of model B (Table III) and model D (Table V) are projected onto the $\left(\alpha_{2}, \alpha_{\kappa}\right)$ plane (right panel); results for model D are equivalent to those of model B. Note that the topology of the projected RG flows in all models is identical.

dimensionless couplings. $\mathrm{FP}_{4}$ corresponds to a line of fixed points (see Table II), which arises from a degeneracy among the $\mathrm{GY}_{12 y}, \mathrm{GY}_{12 y \mathrm{x}^{\prime}}$, and $\mathrm{GY}_{12 \kappa^{\prime}}$ fixed points. The degeneracy is not protected and lifted by higher-loop effects. The gauge-Yukawa fixed points $\mathrm{FP}_{5}-\mathrm{FP}_{8}$ are candidates for UV fixed points. They invariably involve a nonvanishing fixed point for the hypercharge coupling $\alpha_{1}^{*}$, with or without a nonvanishing $\alpha_{2}^{*}$, and fixed points for the Yukawas. We also note that some of the fixed point couplings are of order unity, in particular the hypercharge coupling. Ultimately, this is a consequence of a low number of BSM fermions and the present approximation. We come back to this aspect in Sec. IV where the quartic scalar couplings are retained as well.

Fixed point candidates other than those given in Table II either vanish or come out unphysical. For example, the relation $\beta_{y} / \alpha_{y}=\beta_{\kappa^{\prime}} / \alpha_{\kappa^{\prime}}+2 \alpha_{\kappa}$, which holds in model $\mathrm{A}$ [see (14) and Sec. A for the RG coefficients], implies that at least one of the couplings $\alpha_{y}^{*}$ or $\alpha_{\kappa^{\prime}}^{*}$ has to vanish provided that $\alpha_{\kappa}^{*} \neq 0$. It follows that fixed points such as $\mathrm{GY}_{1 \kappa \kappa^{\prime} y}$ and $\mathrm{GY}_{12 \kappa \kappa^{\prime} y}$ cannot arise. For $\alpha_{\kappa}=0$, we find a line of fixed points in the coupling $\alpha_{y}^{\prime}=\alpha_{y}+\alpha_{\kappa}$. Note also that $\tilde{\alpha}_{y}=$ $\alpha_{y}-c \alpha_{\kappa^{\prime}}$ with $c \neq 1$ a free parameter is decoupled from the rest of the system. The fixed points $\mathrm{GY}_{2 y}, \mathrm{GY}_{2 y \kappa^{\prime}}$, and $\mathrm{GY}_{2 \kappa^{\prime}}$ which are covered by this line of fixed points, are unphysical. As the Yukawa beta functions do not receive vertex corrections, they can be rewritten as $\beta_{y^{\prime}}=$ $\gamma_{y^{\prime}}\left(\alpha_{1}, \alpha_{y}^{\prime}\right) \alpha_{y}^{\prime}$ and $\beta_{\tilde{y}}=\gamma_{y^{\prime}}\left(\alpha_{1}, \alpha_{y}^{\prime}\right) \tilde{\alpha}_{y}$ in terms of a single anomalous dimension $\gamma_{y^{\prime}}$, which, moreover, is independent of $\tilde{\alpha}_{y}$. Therefore, $\tilde{\alpha}_{y}$ becomes exactly marginal for $\gamma_{y^{\prime}}\left(\alpha_{1}^{*}, \alpha_{y}^{\prime *}\right)=0$, and the parameter $c$ remains unspecified. Lines of fixed points related to the vanishing of anomalous dimensions are well-known in supersymmetric gauge theories. Here, they are an artifact of the low orders in

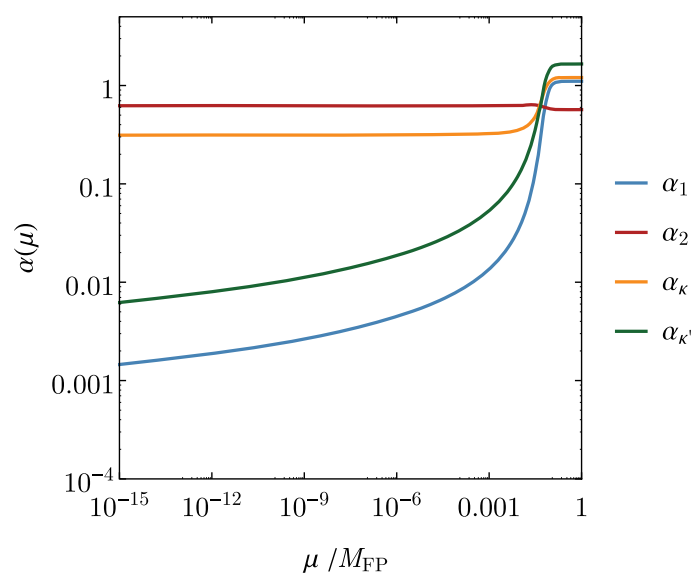

FIG. 2. Running of couplings of model A in the 210 approximation from fixed point $A_{2}$. Trajectories are invariably attracted by $\mathrm{FP}_{3}$ in the infrared, and $\alpha_{2}$ comes out too large compared to the $\mathrm{SM}$ value.

the loop expansion. Finally, we note that the fixed points $\mathrm{GY}_{1 \kappa}$ and $\mathrm{GY}_{12 \kappa}$ arise with negative $\alpha$ which is unphysical.

In Fig. 1, we show the schematic phase diagram of model $A$ and the interplay between the UV fixed point $\mathrm{FP}_{3}-\mathrm{FP}_{7}$ (denoted as $A_{1}-A_{4}$ ) in more detail (see also Table II). Trajectories are projected onto the $\left(\alpha_{2}, \alpha_{\kappa^{\prime}}\right)$ plane, and arrows indicate the flow from the UV to the IR. $A_{3}$ is the most relevant UV fixed point. The separatrices responsible for the crossover from $A_{3}$ to $A_{1}$, from $A_{3}$ to $A_{4}$, or from $A_{1}$ to $A_{2}$ relate to the lines $\alpha_{2}=0, \alpha_{\kappa^{\prime}}=0$, or $\alpha_{y}=0$, respectively. $A_{2}$ is the least ultraviolet point only exhibiting $\alpha_{1}$ as a relevant coupling.

Next, we confirm that some of the UV fixed points in Table II can be matched onto the SM. Here, it is worth noting that many renormalization group trajectories are attracted by the fully attractive IR fixed point $G_{2 \kappa}$, corresponding to $\mathrm{FP}_{3}$ in Table II. If so, the gauge coupling $\alpha_{2}$ remains too large to be matched against the SM. In other words, UV initial conditions within the basin of attraction of $\mathrm{FP}_{3}$ cannot be matched onto the SM. In concrete terms, this is the case for any trajectory running out of the fixed point $A_{2}$ or $A_{4}$ (see Fig. 2 for an example). On the other hand, provided that the gauge coupling $\alpha_{2}$ takes sufficiently small values in the vicinity of the UV fixed point, trajectories can avoid the $\mathrm{FP}_{3}$. This is the case for both UV fixed points $A_{1}$ and $A_{3}$. Starting from these, $\alpha_{2}$ remains sufficiently small throughout the entire RG evolution, and matching against the SM possible at a wide range of matching scales between the $\mathrm{TeV}$ and the Planck scale. An example for this is shown in Fig. 3.

Finally, it is noteworthy that, unlike in [11,14], the Yukawa coupling $\alpha_{y}$ can be switched off as it is not required to generate the fixed points $A_{1}$ and $A_{2}$. Instead, the Yukawa couplings $\kappa$ and $\kappa^{\prime}$ are required to enable a fixed point for $\alpha_{1}$. Their predicted low-energy values are 


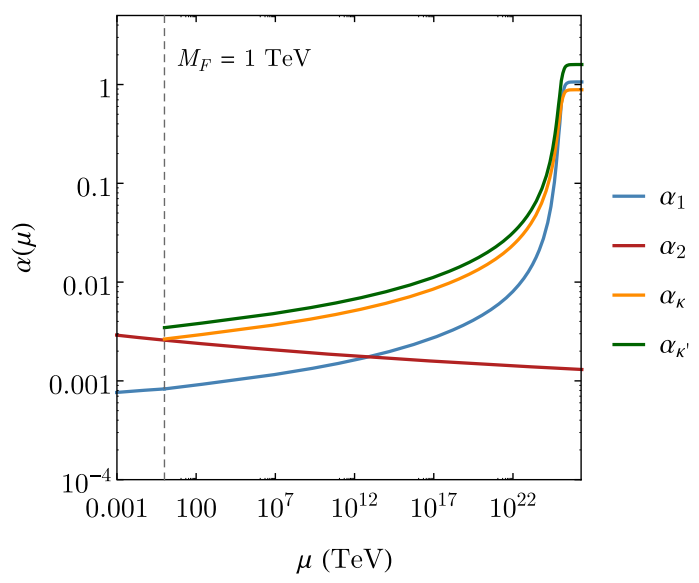

FIG. 3. Running of couplings of model $\mathrm{A}$ in the 210 approximation with matching of the partially interacting fixed point $A_{1}$ to the $\mathrm{SM}$ at $\mu=1 \mathrm{TeV}$ (see Table II).

$\alpha_{\kappa}\left(M_{F}=1 \mathrm{TeV}\right)=2.7 \times 10^{-3}$ and $\alpha_{\kappa^{\prime}}\left(M_{F}=1 \mathrm{TeV}\right)=$ $3.5 \times 10^{-3}$ assuming a matching to $A_{1}$; see Fig. 3 .

\section{B. Model B (triplets, $Y=-1$ )}

For vectorlike fermions $\psi(\mathbf{1}, \mathbf{3},-1)$ the BSM Yukawa Lagrangiean takes the form

$$
-\mathcal{L}_{\mathrm{Y}}^{\mathrm{B}}=+\kappa \bar{L} \psi_{R} H+y \bar{\psi}_{L} S \psi_{R}+\text { H.c. }
$$

The components of $\psi$ can be expressed as the $S U(2)_{L}$ matrix via

$$
\psi=\left(\begin{array}{cc}
\psi^{-1} / \sqrt{2} & \psi^{0} \\
\psi^{-2} & -\psi^{-1} / \sqrt{2}
\end{array}\right),
$$

in accord with the normalization of the kinetic term in Eq. (2). The upper indices indicate the $U(1)_{\mathrm{em}}$ charge of each component.

We have listed all fixed points of model B in Table III. In this model, the one-loop coefficients of both gauge couplings obey $B_{1,2}<0$, turning the Gaussian into a total IR fixed point, and prohibiting any kind of Banks-Zaks solutions. Moreover, all gauge-Yukawa fixed points only involving $\alpha_{2}\left(\mathrm{GY}_{2 \kappa}, \mathrm{GY}_{2 y}, \mathrm{GY}_{2 \kappa y}\right)$ are unphysical, and for the remaining ones, $\alpha_{y}^{*} \neq 0$ is required, additionally excluding $\mathrm{GY}_{1 \kappa}, \mathrm{GY}_{12 \kappa}$.

This singles out the fixed points $B_{1 \ldots 4}$ as listed in Table III. Similar to the fixed points $A_{1 \ldots 4}$ of model A, $B_{2}$ is the least ultraviolet with $\alpha_{1}$ being the only relevant coupling, $B_{1,4}$ are connected to it via a second relevant trajectory, while $B_{3}$ has three relevant directions. This is shown schematically on the right-hand side of Fig. 1. A crucial difference, however, is that no infrared GY fixed points with $\alpha_{2}>0$ and $\alpha_{1}=0$ are realized in model B. Hence, unlike in model A, UV fixed point solutions with finite $\alpha_{2}^{*} \gg \alpha_{2}^{\mathrm{SM}}(\mu \gtrsim 0.1 \mathrm{TeV})$ are not a priori excluded phenomenologically, though constrained, and the corresponding matching conditions $\alpha_{1,2}^{\mathrm{SM}}\left(M_{F}\right)=\alpha_{1,2}^{\mathrm{BSM}}\left(M_{F}\right)$ can have solutions. Integrating the $\mathrm{RG}$ trajectories which leave the $B_{2} \mathrm{UV}$ fixed point into the $\alpha_{1}$ direction toward lower energies, we find $M_{F} \sim 0.025 \mathrm{TeV}$, as depicted in Fig. 4. Similarly, for the fixed point $B_{4}$ we find $M_{F}=\mathcal{O}\left(10^{-2} \mathrm{TeV}\right)$. We learn that asymptotic safety can predict the mass scale of new physics. The scale is disfavored phenomenologically, though only narrowly. The impact of higher-loop corrections is studied in the following Sec. IV.

Fixed point solutions $B_{1,3}$ with $\alpha_{2}^{*}=0$ require more detailed analysis, as asymptotic freedom is absent. Although $\alpha_{2}$ is relevant at the fixed points $B_{1,3}$ due to the Yukawa interactions, it may turn irrelevant along a trajectory toward the IR, as $\alpha_{\kappa, y}$ become smaller causing $B_{2}^{\text {eff }}$ to become negative.

\section{Model C (doublets, $Y=-\frac{1}{2}$ )}

For model C, the BSM fermions have the representation $\psi\left(1,2,-\frac{1}{2}\right)$, which is the same as the one of the SM leptons $L$, leading to the Yukawa interactions

$$
-\mathcal{L}_{\mathrm{Y}}^{\mathrm{C}}=\kappa \bar{E} H^{\dagger} \psi_{L}+\kappa^{\prime} \bar{L} S \psi_{R}+y \bar{\psi}_{L} S \psi_{R}+\text { H.c. }
$$

All physical fixed points in the 210 approximation are listed in Table IV and have $\alpha_{1}$ as an irrelevant coupling. Besides the Gaussian $\left(\mathrm{FP}_{1}\right)$, one Banks-Zaks $\left(\mathrm{FP}_{2}\right)$ and four gauge-Yukawa fixed points in $\alpha_{2}\left(\mathrm{FP}_{3 \ldots 6}\right)$ are realized. Similar to the arguments used in the discussion of model A,

TABLE III. Partially and fully interacting fixed points of model B in the 210 approximation, notation as in Table II. Banks-Zaks fixed points are absent since asymptotic freedom is lost in both gauge couplings; see Figs. 1 and 4 for the phase diagram and sample trajectories.

\begin{tabular}{lccccccccc}
\hline \hline Model B & $\alpha_{1}^{*}$ & $\alpha_{2}^{*}$ & $\alpha_{\kappa}^{*}$ & $\alpha_{y}^{*}$ & Rel. & Irrel. & Info & Fig. 1 & Matching \\
\hline $\mathrm{FP}_{1}$ & $0^{(+)}$ & $0^{(+)}$ & $0^{(+)}$ & $0^{(+)}$ & 0 & 4 & $\mathrm{G}$ & & \\
$\mathrm{FP}_{2}$ & 1.953 & $0^{(-)}$ & 1.562 & 1.888 & 2 & 2 & $\mathrm{GY}_{1 \kappa y}$ & $B_{1}$ & $\checkmark$ \\
$\mathrm{FP}_{3}$ & 1.224 & 0.186 & 1.326 & 1.541 & 1 & 3 & $\mathrm{GY}_{12 \kappa y}$ & $B_{2}$ & $\checkmark$ \\
$\mathrm{FP}_{4}$ & 2.712 & $0^{(-)}$ & $0^{-}$ & 2.712 & 3 & 1 & $\mathrm{GY}_{1 y}$ & $B_{3}$ & $\checkmark$ \\
$\mathrm{FP}_{5}$ & 1.732 & 0.216 & $0^{-}$ & 2.164 & 2 & 2 & $\mathrm{GY}_{12 y}$ & $B_{4}$ & $\checkmark$ \\
\hline \hline
\end{tabular}



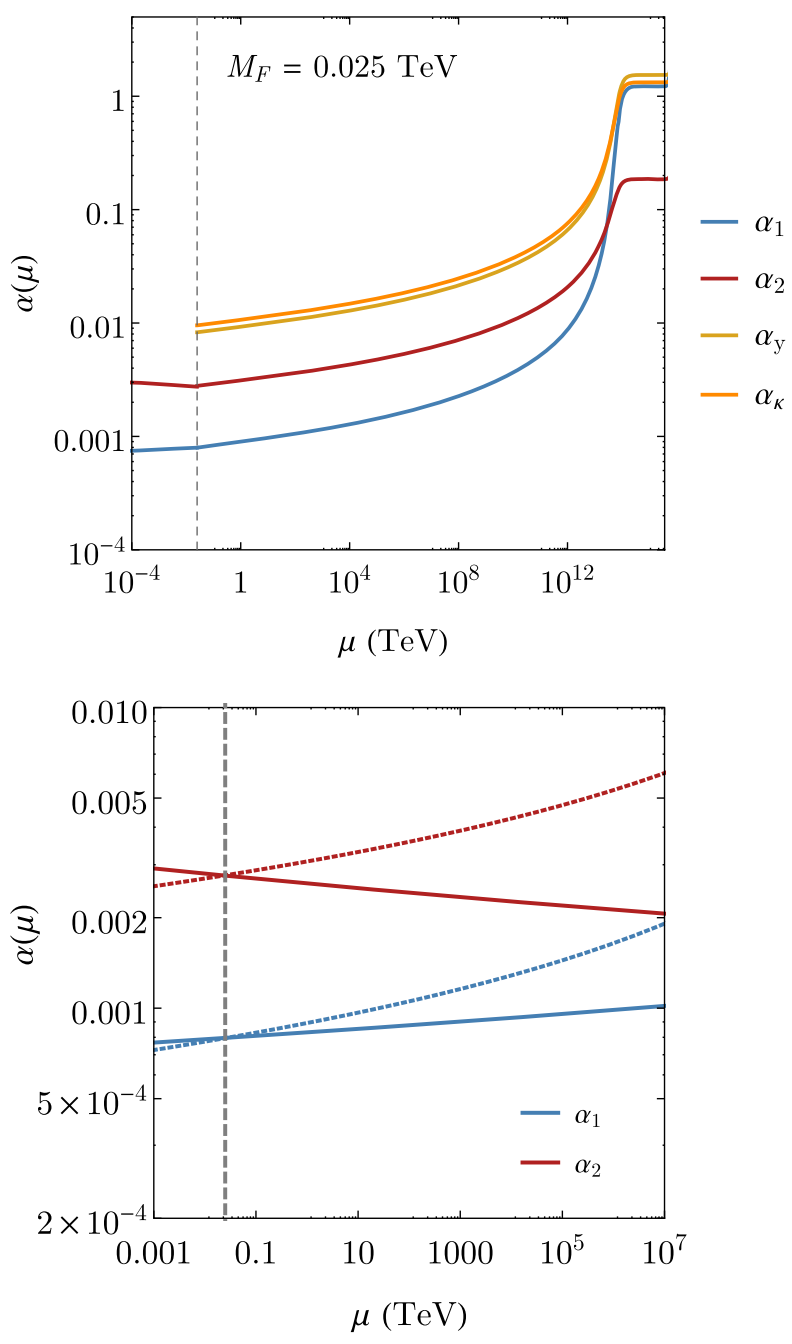

FIG. 4. Matching at $M_{F}=0.025 \mathrm{TeV}$ for the fully interacting fixed point $B_{2}$ of model B. Top panel: BSM running of the couplings into the fixed point. Bottom: BSM (dotted lines) and SM running (solid lines) of the gauge coupling near the matching scale (dashed vertical line).

the relation $\beta_{y} / \alpha_{y}=\beta_{\kappa}^{\prime} / \alpha_{\kappa^{\prime}}+\alpha_{\kappa}$, which holds in model $\mathrm{C}$ [see (14) and Sec. A for the RG coefficients], excludes a solution $\mathrm{GY}_{2 \kappa \kappa^{\prime} y}$. In addition, there is a line of fixed points

TABLE IV. Partially and fully interacting fixed points of model $\mathrm{C}$ in the 210 approximation, with notation as in Table II. At this loop order, no viable candidates for UV fixed points exist.

\begin{tabular}{lcccccccc}
\hline \hline Model C & $\alpha_{1}^{*}$ & $\alpha_{2}^{*}$ & $\alpha_{\kappa}^{*}$ & $\alpha_{\kappa^{\prime}}^{*}$ & $\alpha_{y}^{*}$ & Rel. & Ir. & Info \\
\hline $\mathrm{FP}_{1}$ & $0^{(+)}$ & $0^{(-)}$ & $0^{(+)}$ & $0^{(+)}$ & $0^{(+)}$ & 1 & 4 & $\mathrm{G}$ \\
$\mathrm{FP}_{2}$ & $0^{(+)}$ & 0.038 & $0^{-}$ & $0^{-}$ & $0^{-}$ & 3 & 2 & $\mathrm{BZ}_{2}$ \\
$\mathrm{FP}_{3}$ & $0^{(+)}$ & 0.039 & 0.020 & $0^{-}$ & $0^{-}$ & 2 & 3 & $\mathrm{GY}_{2 \kappa}$ \\
$\mathrm{FP}_{4}$ & $0^{(+)}$ & 0.054 & 0.027 & 0.049 & $0^{+}$ & 0 & 5 & $\mathrm{GY}_{2 \kappa \kappa^{\prime}}$ \\
$\mathrm{FP}_{5}$ & $0^{(+)}$ & 0.053 & 0.011 & $0^{-}$ & 0.046 & 1 & 4 & $\mathrm{GY}_{2 \kappa y}$ \\
$\mathrm{FP}_{6}$ & $0^{(+)}$ & 0.052 & $0^{-}$ & $0.047-\alpha_{y}^{*}$ & $\alpha_{y}^{*}$ & 1 & 3 & $\mathrm{GY}_{2 \kappa^{\prime} y}$ \\
\hline \hline
\end{tabular}

$\alpha_{\kappa^{\prime}}^{*}+\alpha_{y}^{*} \approx 0.047$ with $\alpha_{\kappa}^{*}=0\left(\mathrm{FP}_{6}\right)$ that covers three solutions $\mathrm{GY}_{2 \kappa^{\prime}}, \mathrm{GY}_{2 y}$, and $\mathrm{GY}_{2 \kappa^{\prime} y}$, and gives rise to a marginal coupling. However, no physical gauge-Yukawa fixed point involving $\alpha_{1}$ exists, and hence there is no candidate UV fixed point provided by model $\mathrm{C}$ at lowest loop order.

\section{Model D (doublets, $Y=-\frac{3}{2}$ )}

In model D the BSM Yukawa Lagrangian reads

$$
-\mathcal{L}_{\mathrm{Y}}^{\mathrm{D}}=y \bar{\psi}_{L} S \psi_{R}+\kappa \bar{E} \tilde{H}^{\dagger} \psi_{L}+\text { H.c. },
$$

with $\psi(\mathbf{1}, \mathbf{2},-3 / 2)$. Physical fixed points are listed in Table V, with remarkable small coupling values $\alpha^{*}<1$. All solutions $\alpha_{1}^{*}=0$ suffer from the triviality problem. Besides the Gaussian, and $\mathrm{BZ}_{2}$, all three possible gaugeYukawa fixed points involving $\alpha_{2}$ only are realized $\left(\mathrm{FP}_{3 \ldots 5}\right.$ in Table V), but fall in this category. Viable candidates $D_{1 \ldots 4}$ for UV fixed points are of the gauge-Yukawa type involving at least the $\alpha_{1}$ gauge coupling as well as the BSM Yukawa interaction $\alpha_{y}$, as only $\mathrm{GY}_{1 \kappa}$ and $\mathrm{GY}_{12 \kappa}$ are unphysical.

Projecting onto the $\alpha_{2}-\alpha_{\kappa}$-plane, the hierarchy is similar to model A (see Fig. 1) with $D_{3}$ being the most and $D_{2}$ the least ultraviolet fixed points. Moreover, the same argument holds regarding the total IR fixed point $\mathrm{GY}_{2 \kappa y}$, which attracts trajectories going toward SM coupling values such as those following the $\alpha_{1}$ critical direction from $D_{2,4}$, as depicted on the left-hand side in Fig. 5. Small values of $\alpha_{2}$ along the trajectory are required, implying solutions $D_{1,3}$ as possible UV fixed points. Matching onto the SM is then possible at a range of scales; for $D_{1}$ we obtain $\alpha_{\kappa}\left(M_{F}=1 \mathrm{TeV}\right)=4.2 \times 10^{-3}, \alpha_{y}\left(M_{F}=1 \mathrm{TeV}\right)=5.8 \times 10^{-3}$, which is shown in Fig. 5. Fixed point $D_{3}$ has also been studied in [34], but discarded after including higher order contributions. We retain this fixed point solution, deferring the discussion of higher-loop-order effects to Sec. IV.

\section{E. Model E (singlets, $Y=0$ )}

The Yukawa interactions in model E read

$$
-\mathcal{L}_{\mathrm{Y}}^{\mathrm{E}}=\kappa \bar{L} \tilde{H} \psi_{R}+y \bar{\psi}_{L} S \psi_{R}+\text { H.c. }
$$

Since $\psi$ is a singlet under all gauge groups, $\beta_{y}$ is always positive in the 210 approximation, requiring $\alpha_{y}=0$ at all scales, as this coupling is irrelevant. This decouples the leftchiral BSM fermion $\psi_{L}$ and the BSM scalar $S$ from the SM plus $\psi_{R}$ at this loop order. Only the Gaussian fixed point, the Banks-Zaks in $\alpha_{2}$ and a gauge-Yukawa $\mathrm{GY}_{2 \kappa}$ are present, and $\alpha_{1}$ is irrelevant for all of them. This leaves the model without viable candidates of UV fixed points at 210 approximation. 
TABLE V. Partially and fully interacting fixed points of model D in the 210 approximation, notation as in Table II; see Figs. 1 and 5 for the phase diagram and sample trajectories.

\begin{tabular}{|c|c|c|c|c|c|c|c|c|c|}
\hline Model D & $\alpha_{1}^{*}$ & $\alpha_{2}^{*}$ & $\alpha_{\kappa}^{*}$ & $\alpha_{y}^{*}$ & Rel. & Irrel. & Info & Name & Matching \\
\hline $\mathrm{FP}_{1}$ & $0^{(+)}$ & $0^{(-)}$ & $0^{(+)}$ & $0^{(+)}$ & 1 & 3 & G & & \\
\hline $\mathrm{FP}_{2}$ & $0^{(+)}$ & 0.038 & $0^{-}$ & $0^{-}$ & 2 & 2 & $\mathrm{BZ}_{2}$ & & \\
\hline $\mathrm{FP}_{3}$ & $0^{(+)}$ & 0.039 & 0.020 & $0^{-}$ & 1 & 3 & $\mathrm{GY}_{2 \kappa}$ & & \\
\hline $\mathrm{FP}_{4}$ & $0^{(+)}$ & 0.052 & $0^{-}$ & 0.047 & 1 & 3 & $\mathrm{GY}_{2 y}$ & & \\
\hline $\mathrm{FP}_{5}$ & $0^{(+)}$ & 0.053 & 0.011 & 0.046 & 0 & 4 & $\mathrm{GY}_{2 \kappa y}$ & & \\
\hline $\mathrm{FP}_{6}$ & 0.246 & $0^{(-)}$ & 0.322 & 0.631 & 2 & 2 & $\mathrm{GY}_{1 \kappa y}$ & $D_{1}$ & $\checkmark$ \\
\hline $\mathrm{FP}_{7}$ & 0.202 & 0.145 & 0.295 & 0.647 & 1 & 3 & $\mathrm{GY}_{12 \kappa y}$ & $D_{2}$ & $x$ \\
\hline $\mathrm{FP}_{8}$ & 0.288 & $0^{(-)}$ & $0^{-}$ & 0.778 & 3 & 1 & $\mathrm{GY}_{1 y}$ & $D_{3}$ & $\checkmark$ \\
\hline $\mathrm{FP}_{9}$ & 0.239 & 0.152 & $0^{-}$ & 0.782 & 2 & 2 & $\mathrm{GY}_{12 y}$ & $D_{4}$ & $x$ \\
\hline
\end{tabular}

\section{F. Model F (triplets, $Y=0$ )}

In model $\mathrm{F}$, the BSM fermions $\psi(1,3,0)$ are in the adjoint of $S U(2)_{L}$ with vanishing hypercharge. The BSM Yukawa sector can be written as
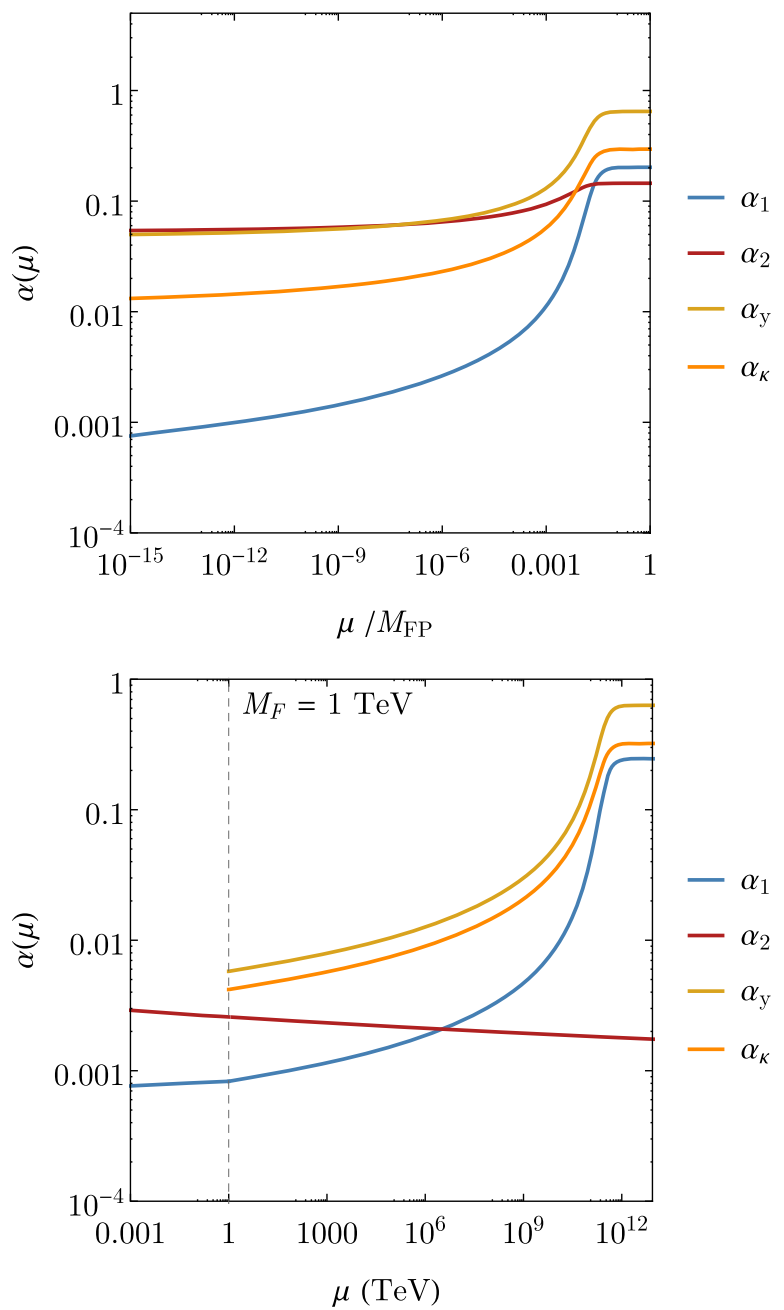

FIG. 5. Renormalization group running of model D. Top: BSM running from fixed point $D_{2}$, where matching is not possible. Bottom: running to the fixed point $D_{1}$ after matching at $\mu=1 \mathrm{TeV}$ (dashed vertical line).

$$
-\mathcal{L}_{\mathrm{Y}}^{\mathrm{F}}=\kappa \bar{L} \tilde{H} \psi_{R}+y \bar{\psi}_{L} S \psi_{R}+\text { H.c. }
$$

In this setup, asymptotic freedom is absent for both gauge couplings, making the Gaussian completely IR attractive and excluding any kind of Banks-Zaks fixed points. In the 210 approximation, $\beta_{1}$ is independent of $\alpha_{y}$, and $\beta_{y}$ is independent of $\alpha_{1}$, as $\psi$ does not carry hypercharge. Hence the two-loop contributions of $\kappa$ are the only negative terms in $\beta_{1}$, requiring $\alpha_{\kappa}^{*} \neq 0$. Moreover, only $\alpha_{2}$ contributions are negative in $\beta_{y}$, which suggests that $\alpha_{2}^{*}=0$ implies $\alpha_{y}^{*}=0$ and irrelevant. However, none of the remaining gaugeYukawa solutions $\mathrm{GY}_{1 \kappa}, \mathrm{GY}_{2 \kappa}, \mathrm{GY}_{2 \kappa y}, \mathrm{GY}_{12 \kappa}$, and $\mathrm{GY}_{12 \kappa y}$ are realized, as $\kappa$ contributions in $\beta_{1}$ are too small compared to one- and other two-loop terms. This leaves the Gaussian as the only physical fixed point; we conclude that there is no AS fixed point at 210 in model $\mathrm{F}$.

\section{G. Summary top down}

In Secs. III A-III F we have gained first insights into the fixed point structure of models $\mathrm{A}-\mathrm{F}$ in a top-down approach of solving the renormalization group equations (RGEs) at leading orders directly and running toward infrared scales. The results for models A, B, and D collected in Tables II, III, and V show several signatures of UV fixed points that can be matched onto the SM, but also indicate that those are borderline perturbative. This suggests that the fixed points are sensitive to contributions from higher-loop orders. We also found that the models $\mathrm{C}$, $\mathrm{E}$, and $\mathrm{F}$ do not provide any viable solutions at 210 and the question arises whether this is just a feature of the approximation. In order to address both points, we go in Sec. IV beyond the 210 approximation. To handle the increased algebraic complexity of higher-loop corrections and the quartic sector, a bottom-up approach will be employed, studying the RG running from the IR to the UV instead, mapping out the BSM critical surface.

\section{RUNNING COUPLINGS}

In this section, we discuss the renormalization group flow of couplings beyond the leading order approximation 
which has been employed in the previous Sec. III. We explore in detail how the running of couplings depends on the values of BSM couplings $\left\{\alpha_{\mathrm{BSM}}\right\}$ at the matching scale. The main new technical additions in this section are the quartic scalar and the portal couplings, and the inclusion of loop effects up to the complete two-loop order (222 approximation) or, if available, the complete three-loop order (333 approximation). We are particularly interested in the running of couplings from a bottom-up perspective, and we study the flow for a given set of BSM initial values $\alpha_{\mathrm{BSM}}$ at the matching scale. We then ask whether these values together with the SM input reach Planckian energies without developing poles, exhibit asymptotic safety, and have the stability of the quantum vacuum.

We give our setup and initial conditions in Sec. IV A, and briefly review the RG flow within the SM in Sec. IV B. After identifying relevant correlations between feeble and weakly sized BSM couplings in Secs. IV C and IV D, respectively, we present in Sec. IVE the BSM critical surface for each model.

\section{A. Setup and boundary conditions}

We retain the renormalization group running for the three gauge couplings of the SM (11), and up to three BSM Yukawa couplings (12). Going beyond the leading order 210 approximation, we also retain the Higgs quartic selfinteraction $\lambda$, the BSM quartics $u, v$, and the quartic portal coupling $\delta$

$$
\begin{array}{lll}
\alpha_{\lambda}=\frac{\lambda}{(4 \pi)^{2}}, & \alpha_{\delta}=\frac{\delta}{(4 \pi)^{2}}, \\
\alpha_{u}=\frac{u}{(4 \pi)^{2}}, & \alpha_{v}=\frac{v}{(4 \pi)^{2}} .
\end{array}
$$

Moreover, it is well-known that the SM top and bottom Yukawa couplings $y_{t, b}$ critically influence the running of the Higgs quartic and, therefore, must be retained as well. We introduce them as

$$
\alpha_{t}=\frac{y_{t}^{2}}{(4 \pi)^{2}}, \quad \alpha_{b}=\frac{y_{b}^{2}}{(4 \pi)^{2}} .
$$

Overall, Eqs. (11), (12), (36), and (37) result in 12 (or 11) independent running couplings for models $\mathrm{A}$ and $\mathrm{C}$ (or models $\mathrm{B}, \mathrm{D}, \mathrm{E}$, and F).

We also remark that the scalar quartic interactions couple back into the Yukawa sectors starting at two-loop, and into the gauge sectors starting at three- (or four-) loop, depending on whether the participating matter fields are charged (uncharged) under the gauge symmetry. Conversely, the Yukawa couplings couple back into the quartic starting at one-loop, as do the weak and hypercharge gauge couplings into the Higgs. We expect therefore a crucial interplay between BSM Yukawas and the portal coupling with Higgs stability. In addition, the leading order study in Sec. III showed that some of the fixed point coordinates might come out within the range $\mathcal{O}(0.1-1.0)$, indicating that strict perturbativity cannot be guaranteed. For these reasons, we develop the fixed point search and the study of RG equations up to the highest level of approximation where all couplings are treated on an equal footing, i.e., the complete two-loop order (222 approximation). The running of SM couplings, which serves as a reference scenario, is studied up to the complete three-loop order (333 approximation).

All our models require boundary conditions with six SM couplings at the matching scale $\mu_{0}$, which for all practical purposes corresponds to the mass of the BSM fermions $\psi$. To be specific, we take the matching scale in this section to be

$$
\mu_{0}=1 \mathrm{TeV} .
$$

The initial conditions for the SM couplings then read, using $M_{t} \simeq 172.9 \mathrm{GeV}$ and $[53,54]$,
$\alpha_{1}\left(\mu_{0}\right) \simeq 8.30 \times 10^{-4}$,
$\alpha_{\lambda}\left(\mu_{0}\right) \simeq 6.09 \times 10^{-4}$
$\alpha_{2}\left(\mu_{0}\right) \simeq 2.58 \times 10^{-3}$,
$\alpha_{t}\left(\mu_{0}\right) \simeq 4.61 \times 10^{-3}$,
$\alpha_{3}\left(\mu_{0}\right) \simeq 7.08 \times 10^{-3}$,
$\alpha_{b}\left(\mu_{0}\right) \simeq 1.22 \times 10^{-6}$.

Hence, in our conventions, initial couplings are within the range $\mathcal{O}\left(10^{-6}-10^{-2}\right)$. We are now in a position to discuss the running of couplings and the "BSM critical surface," i.e., the set of values for BSM couplings at the matching scale which lead to viable RG trajectories all the way up to the Planck scale.

\section{B. Standard model}

We briefly discuss running couplings within the SM at the complete three-loop order in perturbation theory [54-61], displayed in Fig. 6. Overall, the SM running is rather slow with gauge, quartic, and Yukawa couplings mostly below $\mathcal{O}\left(10^{-2}\right)$ or smaller. We also observe that the Higgs potential becomes metastable starting around $10^{10} \mathrm{GeV}[54,55]$, an effect which is mostly driven by the quantum corrections from the top Yukawa coupling $\alpha_{t}$. Further, an imperfect gauge coupling unification is observed around $10^{16} \mathrm{GeV}$. Quantum gravity is expected to kick in around the Planck scale, $M_{\mathrm{Pl}} \approx 10^{19} \mathrm{GeV}$, indicated by the gray-shaded area. As an aside, we notice that the Higgs beta function essentially vanishes at Planckian energies

$$
\mu \approx M_{\mathrm{Pl}}: \quad \alpha_{\lambda} \approx 10^{-4}, \quad \beta_{\lambda} \approx 0 .
$$

If quantum gravity can be neglected, hypothetically, we may extend the running of couplings into the transPlanckian regime. The hypercharge coupling would then reach a Landau pole around $10^{41} \mathrm{GeV}$. Also, its slow but steady growth would eventually dominate over the slowly decreasing top Yukawa coupling, and thereby stabilize the quantum vacuum starting around $10^{29} \mathrm{GeV}$. Ultimately, however, the Higgs coupling reaches a Landau pole alongside the $U(1)_{Y}$ coupling and the SM stops being predictive. 


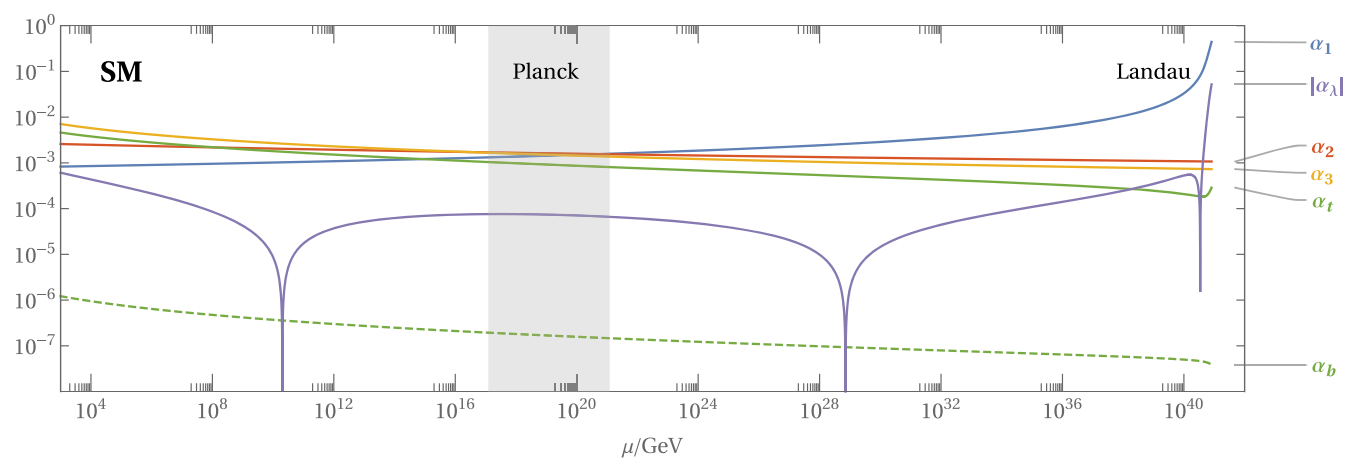

FIG. 6. Renormalization group running of the SM. Shown are the gauge, Higgs, top (solid green line) and bottom (dashed green line) Yukawa couplings at the complete three-loop order starting from the $1 \mathrm{TeV}$ regime up to the deep UV. The Planck scale is indicated by the gray band. The Higgs self-coupling changes sign twice, around $10^{10} \mathrm{GeV}$ and around $10^{30} \mathrm{GeV}$. In between, the SM vacuum is metastable. Ultimately, the hypercharge and the Higgs coupling approach UV Landau poles around $10^{41} \mathrm{GeV}$.

\section{Feeble BSM couplings}

Next, we include new matter fields on top of the SM ones and switch on the BSM couplings at the matching scale (39). A minimally invasive choice are very small, feeble, BSM couplings such that they do not significantly
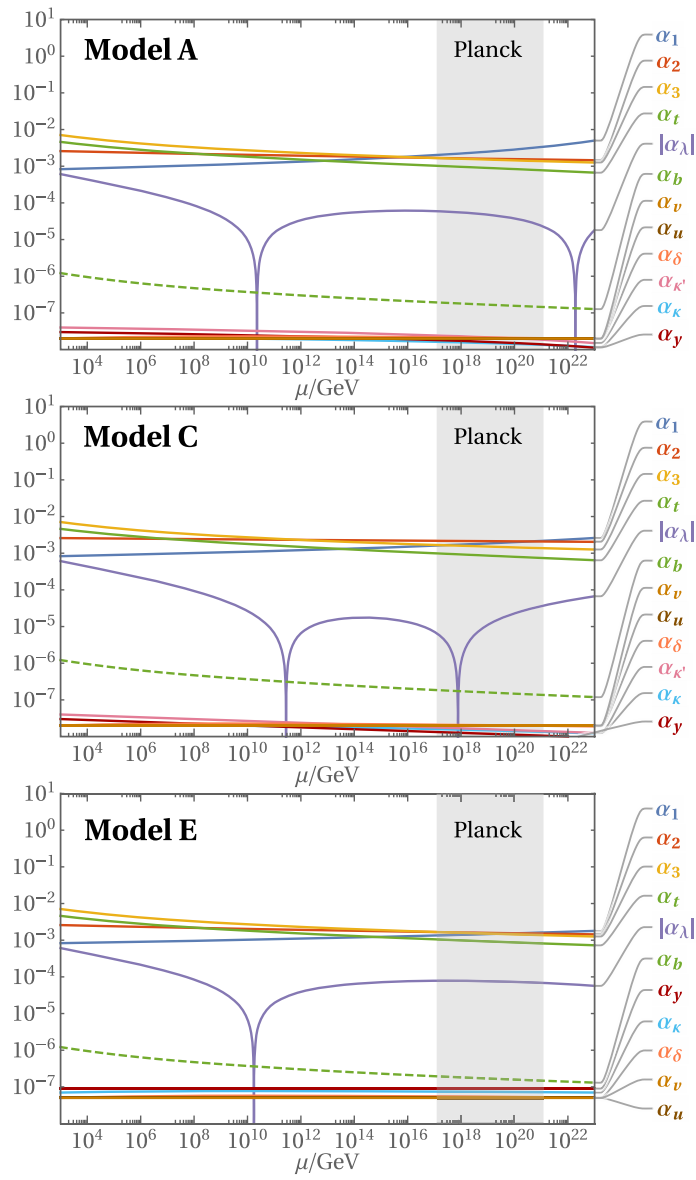

influence the renormalization group flow up to the Planck scale. Their own running would then be well encoded already by the leading order in the perturbative expansion, and models resemble the SM, extended by vectorlike fermions. Specifically, we consider here initial values of the order of $\alpha_{\mathrm{BSM}} \approx 10^{-7}$ or smaller.
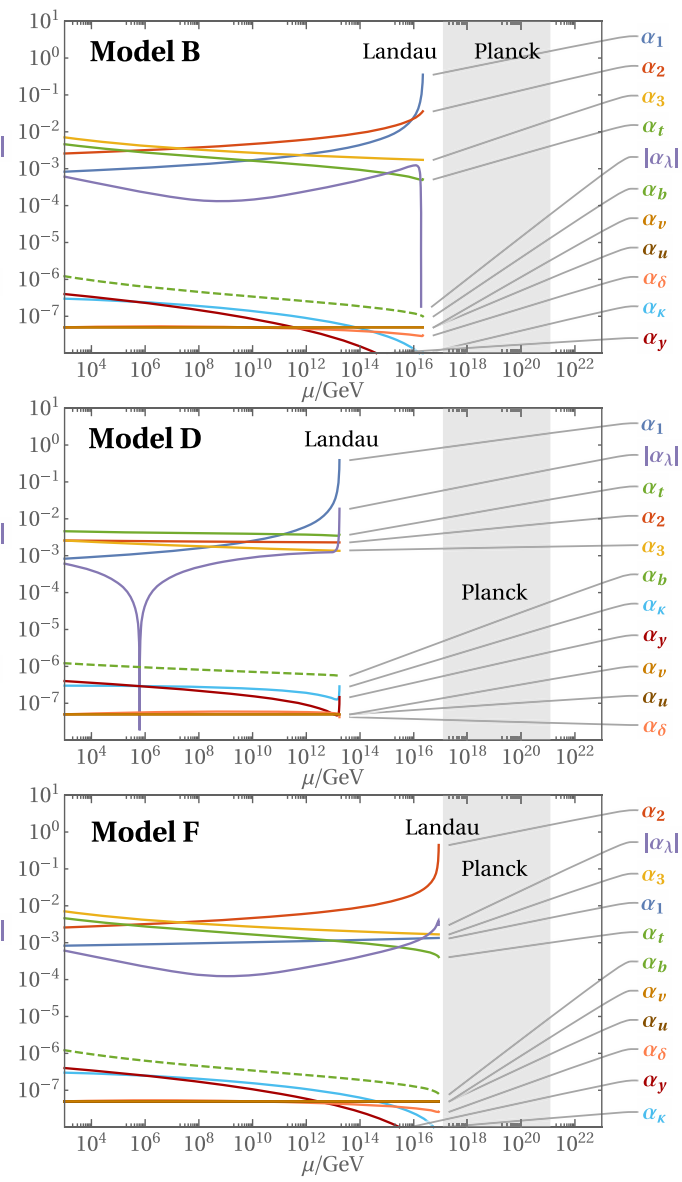

FIG. 7. Renormalization group running of models A-F with feeble BSM couplings. The gray-shaded area indicates the Planck scale. Sub-Planckian Landau poles arise in models B and D (model F) in the hypercharge (weak) coupling. 

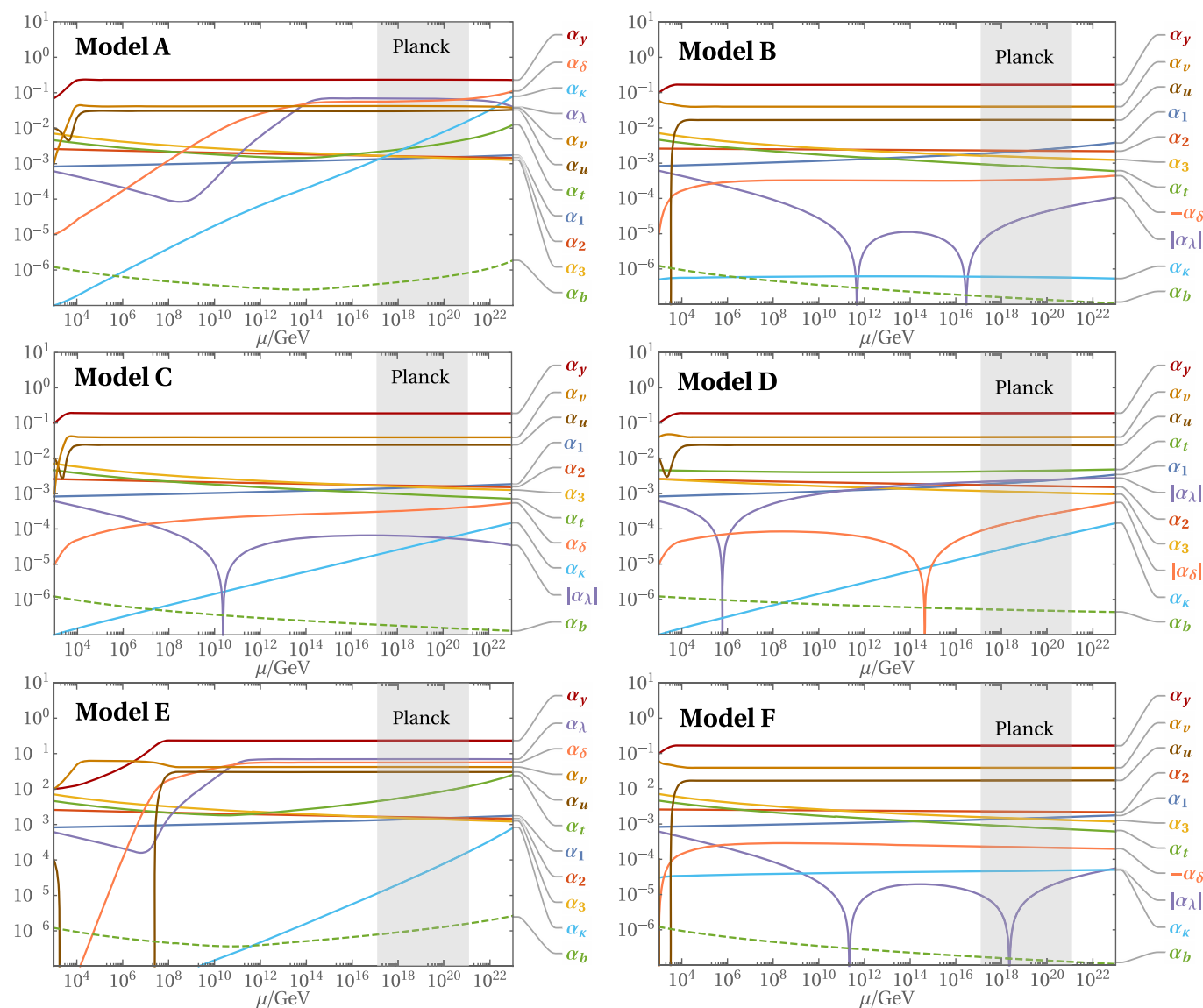

FIG. 8. Renormalization group running of models A-F and $\alpha_{\kappa} \approx 0,\left|\alpha_{\delta}\right|=10^{-5}$ (except for model E, where $\left|\alpha_{\delta}\right|$ is very feeble), as well as $\alpha_{\kappa^{\prime}}=0$ for models A and C. In models A, C, D, and E, small initial values of $\alpha_{\kappa}$ (light blue line) blow up in the UV, while for B and F the trajectories remain more stable. The Higgs potential (lilac line) is not stabilized by Yukawa interactions (models B, C, F), but for sufficiently large initial values of $\alpha_{\delta}$ (orange line) in the singlet models (models $\mathrm{A}$ and $\mathrm{E}$ ).

\section{Models A, C, and $E$}

Sample trajectories with feeble BSM couplings are shown in Fig. 7 (plots to the left) for models A, C, and E. In all cases, we observe a SM-like running of couplings. The new matter fields modify the running of gauge couplings very mildly. For models $\mathrm{A}$ and $\mathrm{E}$, we find a vanishing beta function for the Higgs quartic coupling, much similar to the SM (40). For model C, we observe that the regime of Higgs metastability terminates exactly around the Planck scale,

$$
\mu \approx M_{\mathrm{Pl}}: \quad \alpha_{\lambda} \approx 0, \quad \beta_{\lambda} \approx 0 .
$$

We conclude that in models A, C, and E feeble initial values for the BSM couplings lead to SM-like trajectories including vacuum metastability up to the Planck scale. Hence, the BSM critical surface covers the region in which all couplings are feeble.

\section{Models B, D, and $F$}

The models B, D, and F with feeble BSM couplings at $\mu_{0}$ reach a Landau pole prior to the Planck scale, with sample trajectories shown in Fig. 7 (plots to the right). Specifically, in model B asymptotic freedom for the weak and hypercharge couplings is lost leading to a Landau pole around $10^{16} \mathrm{GeV}$ reached first for the hypercharge, going hand-in-hand with the loss of vacuum stability. Similarly, a strong coupling regime with a Landau pole is reached around $10^{13} \mathrm{GeV}$ $\left(10^{16} \mathrm{GeV}\right)$ for model D (model F). Hence, none of these models can make it to the Planck scale for feeble BSM couplings, excluding this region from the BSM critical surface. Notice though that the growth of the gauge couplings in models B and F stabilizes the Higgs sector all the way up to close to the pole.

\section{Weak BSM couplings}

In the following we explore several matching scenarios for each of the models A-F with BSM couplings of at least the same order of magnitude as the SM couplings at the matching scale (39). In this regime, Yukawa interactions play a crucial role in avoiding Landau poles and stabilizing RG flows, inviting a classification by the couplings involved. Because of the importance for Higgs stability, we also distinguish scenarios with or without portal coupling effects. After identifying relevant correlations between BSM 

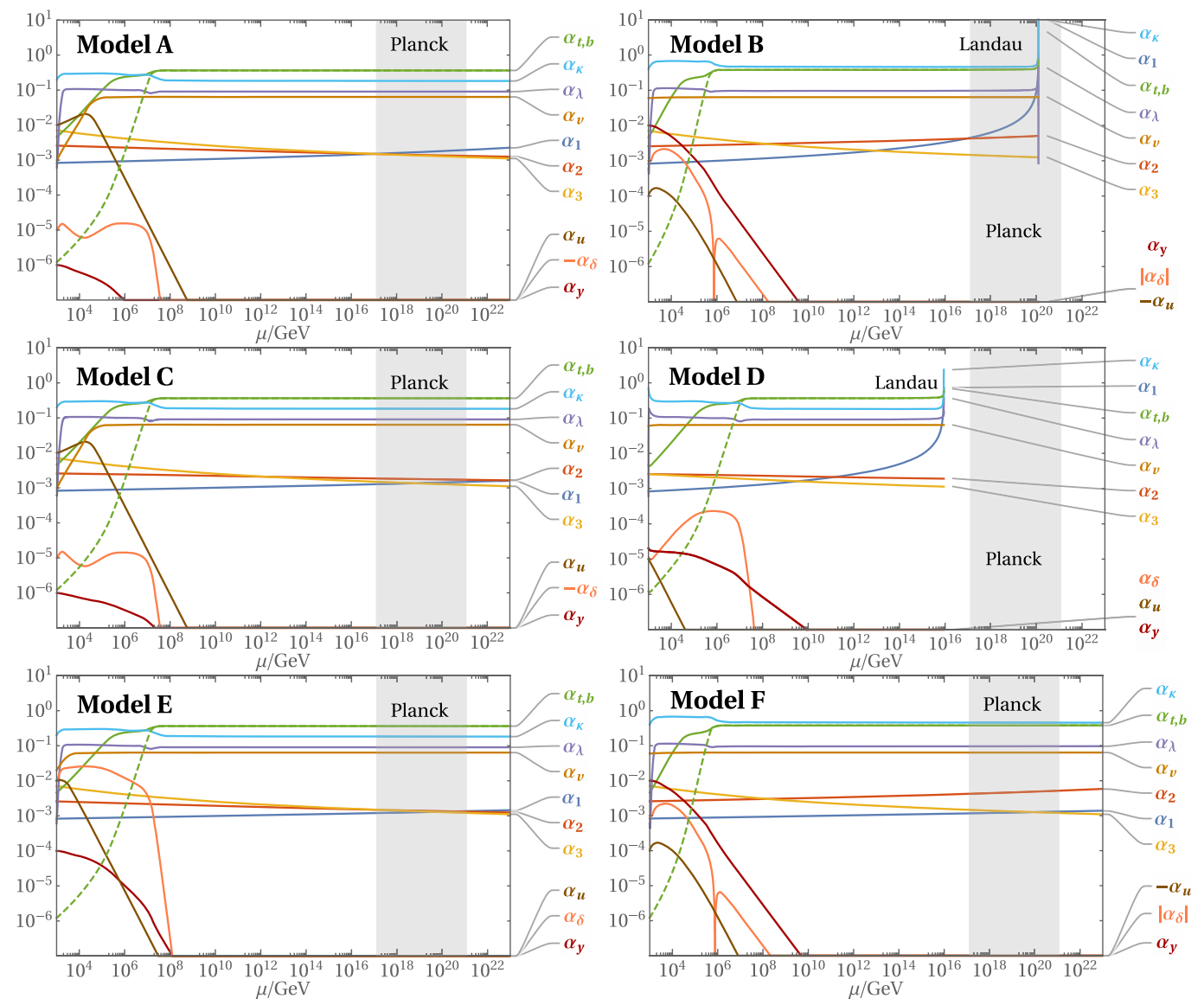

FIG. 9. Renormalization group running of models A-F with $\alpha_{y} \approx 0$ and $\left|\alpha_{\delta}\right| \approx 0$, and $\alpha_{\kappa^{\prime}}=0$ for models A and C. In all models the couplings $\alpha_{y, u, \delta}$ (red, brown, orange lines) are driven to zero in the UV. The solid (dashed) green line denotes the flow of the SM top (bottom) Yukawa, which merge at the crossover.

couplings, we obtain in Sec. IV E the BSM critical surface for each model.

\section{Models $A-F$ with $\alpha_{y} \neq 0$}

For $\alpha_{\kappa, \kappa^{\prime}} \approx 0$, the BSM Yukawa $\alpha_{y} \neq 0$ slows down the running of gauge couplings and removes all Landau poles before $M_{\mathrm{Pl}}$. Moreover, it stabilizes the running of the quartics $\alpha_{u, v}$, due to a walking regime $\beta_{y, u, v} \approx 0$, which may extend until after the Planck scale. This is displayed in Fig. 8. Because of sizable BSM couplings, the portal $\alpha_{\delta}$ is being switched on, influencing the running of the Higgs quartic $\alpha_{\lambda}$. For larger values $\left.\alpha_{\delta}\right|_{M_{F}}$, the Higgs potential can be stabilized, i.e., $\alpha_{\lambda}>0$ between $M_{F}$ and $M_{\mathrm{Pl}}$ (models A and E), while smaller values of $\left.\alpha_{\delta}\right|_{M_{F}}$ cause the Higgs potential to flip sign twice before the Planck scale (models $\mathrm{B}$ and $\mathrm{F}$ ), or $\alpha_{\lambda}$ remains negative at $M_{\mathrm{Pl}}$ (models $\mathrm{C}$ and $\mathrm{D}$ ).

In models A, C, D, and E, feeble initial values of $\alpha_{\kappa}$ grow in coupling strength, eventually destabilizing the trajectories in the far UV. For the triplet models B and F, $\beta_{\kappa}$ remains small for feeble or weakly coupled $\alpha_{\kappa}$, providing greater windows of stability. In summary, the BSM critical surface covers the parameter space where $\alpha_{y}$ is weak and $\alpha_{\kappa, \kappa^{\prime}}$ are feeble at the matching scale.

\section{Models $A-F$ with $\alpha_{\kappa} \neq 0$}

A weakly coupled Yukawa interaction $\alpha_{\kappa}$ may stabilize the SM scalar sector. The choice,

$$
\alpha_{y, \kappa^{\prime}}=0, \quad \alpha_{\kappa} \neq 0, \quad\left|\alpha_{\delta}\right| \approx 0,
$$

is depicted in Fig. 9. A common feature of all models A-F is the stabilization of $\alpha_{\lambda}$ in a walking region together with $\alpha_{\kappa}$ and the SM Yukawas, as all of which couple to the SM Higgs directly. The BSM potential, on the other hand, lacks a sizable Yukawa interaction, and $\alpha_{v}$ self-stabilizes around $\alpha_{v}^{*} \approx 13 / 204$. This phenomenon is not disrupted by feeble initial values of $\left|\alpha_{u, y, \delta}\right|$, which are driven to zero in the UV limit. However, the scenario is not viable for model D as the Landau pole still appears before the Planckian regime. In model B, the pole appears soon after $M_{\mathrm{Pl}}$.

The initial value of $\alpha_{\kappa}$ can be reduced for $\left.\alpha_{\delta}\right|_{M_{F}}$ large enough to stabilize the running of the Higgs quartic: 

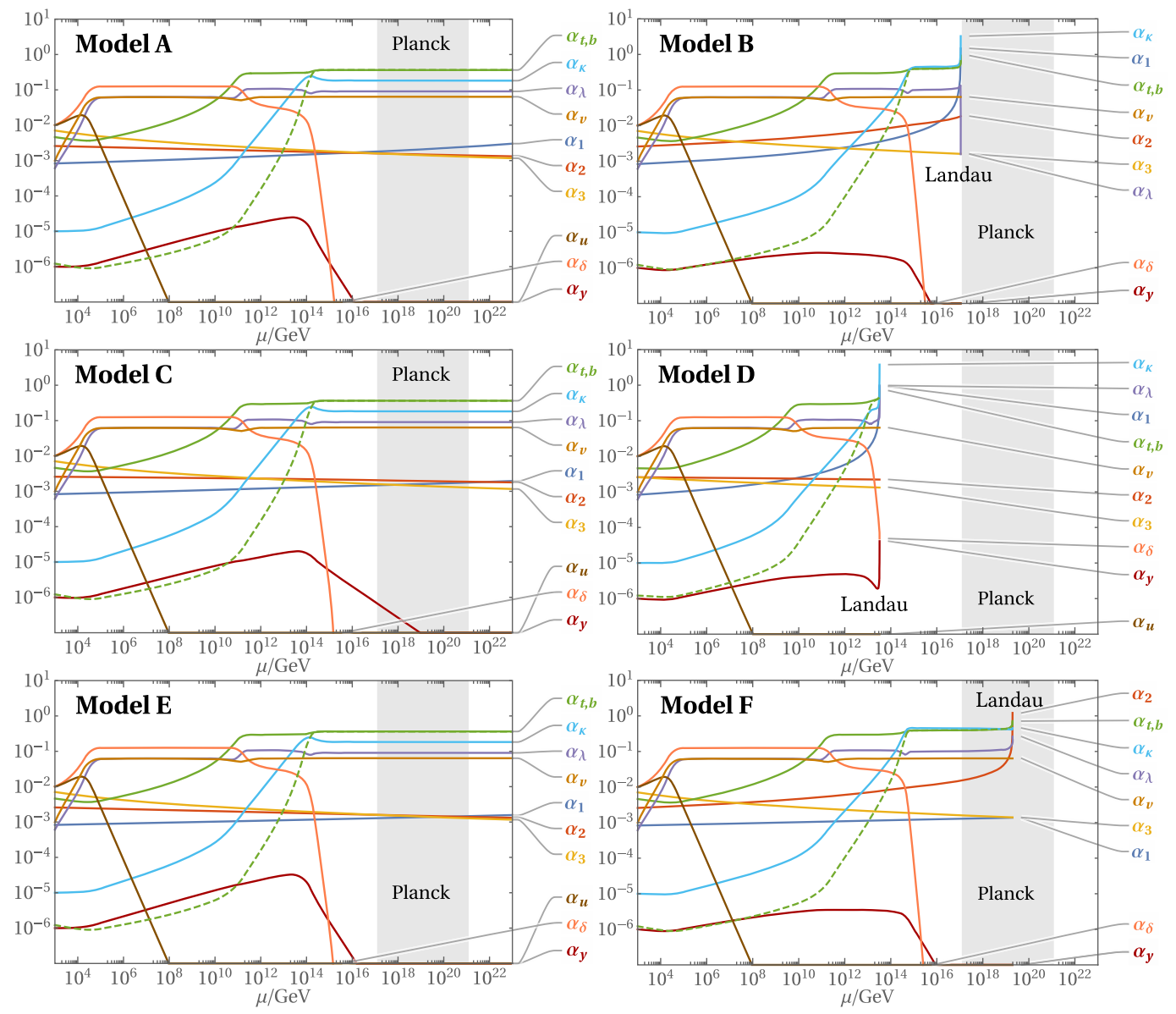

FIG. 10. As in Fig. 9 but for significantly lower values of $\alpha_{\kappa}$ (light blue line) at the matching scale. Models B, D, and F exhibit Landau poles before or at the Planck scale. Qualitative features observed in Fig. 9 for $\alpha_{y, u, \delta}$ (drop toward UV) and $\alpha_{b, t}$ (merging) remain.

$$
\alpha_{y, \kappa^{\prime}}=0, \quad \alpha_{\kappa} \neq 0, \quad\left|\alpha_{\delta}\right| \neq 0 .
$$

For models A, C, and E, this allows for feeble $\alpha_{\kappa}$ at the matching scale, while in models $\mathrm{B}, \mathrm{D}$, and $\mathrm{F}$ poles arise below or at the Planck regime, as displayed in Fig. 10.

\section{Models $A$ and $C$ with $\alpha_{\kappa^{\prime}} \neq 0$}

Models $\mathrm{A}$ and $\mathrm{C}$ feature the additional Yukawa interaction $\alpha_{\kappa^{\prime}}$, giving rise to another walking regime

$$
\alpha_{y}=0, \quad \alpha_{\kappa, \kappa^{\prime}} \neq 0, \quad\left|\alpha_{\delta}\right| \approx 0,
$$

shown in Fig. 11. Starting from the matching scale $M_{F}$, these regions are reached before the Planck scale, and at various speeds by different couplings, creating a rich landscape of intermediate pseudofixed points and scales. Throughout the walking regime, SM and BSM Yukawas and quartics slow down in model $\mathrm{A}$ at

$$
\begin{array}{rlrl}
\alpha_{t, b}^{*} & \simeq 3.61 \times 10^{-1}, & & \alpha_{\kappa^{\prime}}^{*} \simeq 2.32 \times 10^{-1}, \\
\alpha_{\kappa}^{*} \simeq 1.80 \times 10^{-1}, & & \alpha_{u}^{*} \simeq 3.07 \times 10^{-2}, \\
\alpha_{\lambda}^{*} \simeq 8.95 \times 10^{-2}, & & \alpha_{v}^{*} \simeq 4.12 \times 10^{-2},
\end{array}
$$

and in model $\mathrm{C}$ at

$$
\begin{array}{rlrl}
\alpha_{t, b}^{*} & \simeq 3.61 \times 10^{-1}, & & \alpha_{\kappa^{\prime}}^{*} \simeq 1.88 \times 10^{-1}, \\
\alpha_{\kappa}^{*} \simeq 1.80 \times 10^{-1}, & & \alpha_{u}^{*} \simeq 2.44 \times 10^{-2}, \\
\alpha_{\lambda}^{*} \simeq 8.95 \times 10^{-2}, & & \alpha_{v}^{*} \simeq 3.92 \times 10^{-2} .
\end{array}
$$

On the other hand, the portal $\alpha_{\delta}$ and gauge couplings continue to run, although the latter is slowed down by the magnitude of the Yukawas. Consequently, Landau poles are avoided even far beyond the Planck scale. Moreover, the SM [BSM] quartics $\alpha_{\lambda}\left[\alpha_{u}, \alpha_{v}\right]$ are stabilized by the $\alpha_{\kappa}\left[\alpha_{\kappa^{\prime}}\right]$ Yukawa couplings. All of these phenomena are consequences of the vicinity of a pseudofixed point with $\alpha_{1,2,3, y, \delta}^{*}=0$, separating the SM and BSM scalar sectors, as well as Yukawa couplings from each other. This decoupling is expected to be realized to all loop orders, because, in its vicinity, the action decomposes as

$$
\mathcal{S}=\mathcal{S}_{H}\left(H, L[E], \psi_{R[L]}\right)+\mathcal{S}_{S}\left(S, E[L], \psi_{L[R]}\right)
$$

for model A [C], up to corrections of the order of the SM lepton Yukawas $Y_{\ell}$, Eq. (9). However, this separation can 

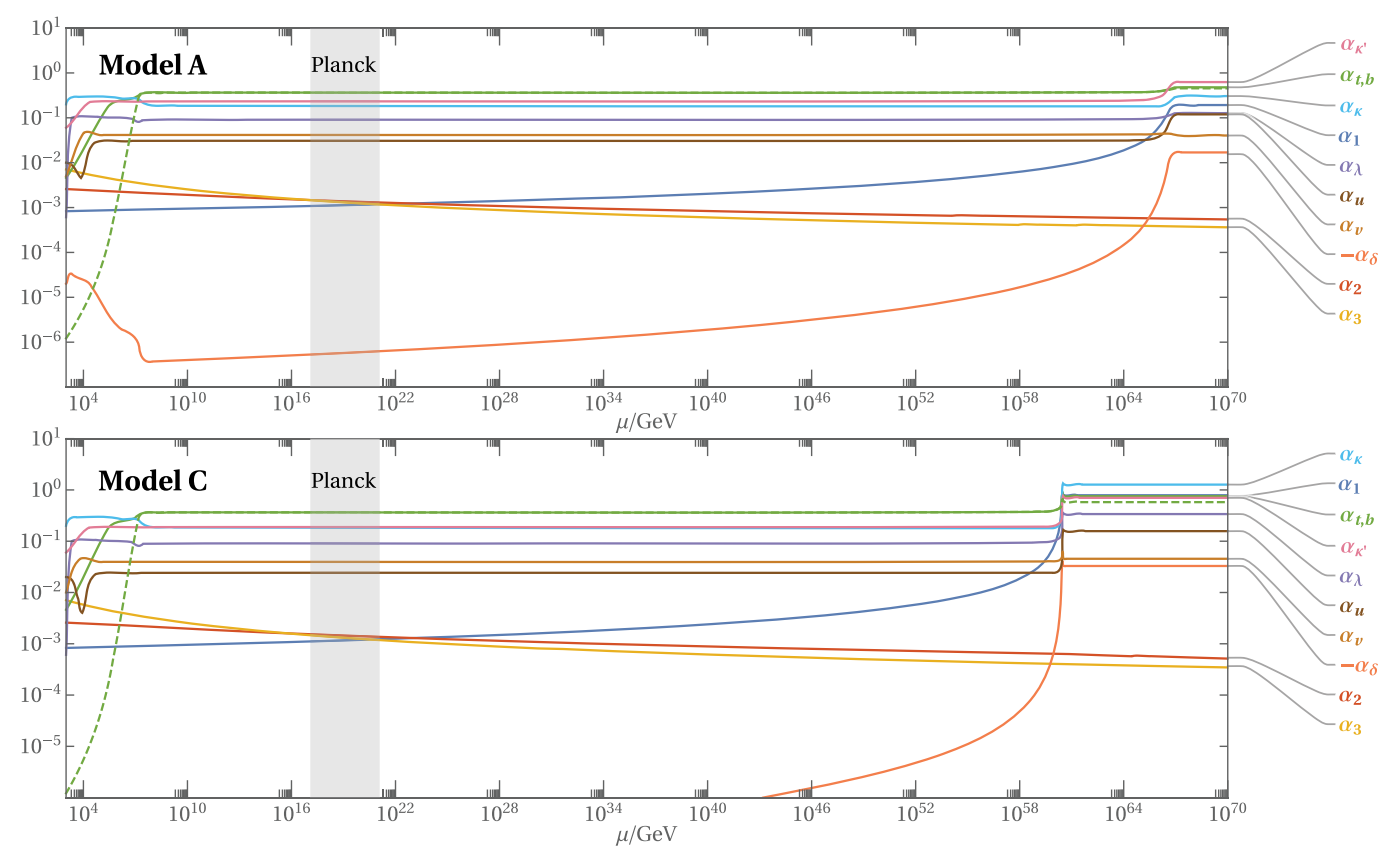

FIG. 11. Renormalization group running in models $\mathrm{A}$ and $\mathrm{C}$ with $\alpha_{y}=0, \alpha_{\kappa, \kappa^{\prime}} \neq 0$, and $\left|\alpha_{\delta}\right| \lesssim 10^{-5}$. The solid (dashed) green line denotes the SM top (bottom) Yukawa. The flow is stabilized by a crossover fixed point just before the Planck scale (gray area); see text. As $\alpha_{1}$ (steel blue line) becomes large, a complete UV fixed point is reached in the far UV.

be realized only approximately for small gauge and portal couplings. Hence, the RG flow eventually leaves the walking regime in the far UV due to the slow residual running of $\alpha_{1}$ or $\alpha_{\delta}$. Ultimately, this triggers a crossover away from the walking regime and into an interacting UV fixed point regime where all couplings bar the non-Abelian gauge and the BSM Yukawa couplings take nontrivial values.

Specifically, for model A, the interacting UV fixed point is approximately given by

$$
\begin{array}{ll}
\alpha_{1}^{*} \simeq 1.93 \times 10^{-1}, & \alpha_{\kappa}^{*} \simeq 3.05 \times 10^{-1}, \\
\alpha_{3}^{*}=\alpha_{2}^{*}=\alpha_{y}^{*}=0, & \alpha_{\kappa^{\prime}}^{*} \simeq 6.25 \times 10^{-1}, \\
\alpha_{\lambda}^{*} \simeq 1.27 \times 10^{-1}, & \alpha_{\delta}^{*} \simeq-1.55 \times 10^{-2}, \\
\alpha_{t}^{*} \simeq 4.78 \times 10^{-1}, & \alpha_{u}^{*} \simeq 1.19 \times 10^{-1}, \\
\alpha_{b}^{*} \simeq 4.53 \times 10^{-1}, & \alpha_{v}^{*} \simeq 4.03 \times 10^{-2} .
\end{array}
$$

Note that the fixed point is rather close to the values of couplings in the walking regime (45). Similarly, in model C we find an approximate UV fixed point with coordinates

$$
\begin{array}{ll}
\alpha_{1}^{*} \simeq 7.64 \times 10^{-1}, & \alpha_{\kappa}^{*} \simeq 3.05 \times 10^{-1}, \\
\alpha_{3}^{*}=\alpha_{2}^{*}=\alpha_{y}^{*}=0, & \alpha_{\kappa^{\prime}}^{*} \simeq 7.00 \times 10^{-1}, \\
\alpha_{\lambda}^{*} \simeq 3.38 \times 10^{-1}, & \alpha_{\delta}^{*} \simeq-3.30 \times 10^{-2}, \\
\alpha_{t}^{*} \simeq 7.51 \times 10^{-1}, & \alpha_{u}^{*} \simeq 1.57 \times 10^{-1}, \\
\alpha_{b}^{*} \simeq 5.76 \times 10^{-1}, & \alpha_{v}^{*} \simeq 4.54 \times 10^{-2} .
\end{array}
$$

Again, we note that (48) is numerically close to the walking regime (46).

Reducing $\left.\alpha_{\kappa}\right|_{M_{F}}$ destabilizes the running of the Higgs self-coupling $\alpha_{\lambda}$, which can, however, be remedied by a nonvanishing portal coupling $\alpha_{\delta}$ :

$$
\alpha_{y}=0, \quad \alpha_{\kappa, \kappa^{\prime}} \neq 0, \quad\left|\alpha_{\delta}\right| \neq 0 .
$$

In model A, this enables trajectories with feeble $\left.\alpha_{\kappa}\right|_{M_{F}}$ to connect to the phenomena (45) and (48), while for model C, trans-Planckian poles arise. This is displayed in Fig. 12. In both models the coupling $\alpha_{u}$ (brown line), whose overall sign separates the vacuum solutions $V^{+}$from $V^{-}$, Eq. (22), changes sign below $M_{\mathrm{Pl}}$.

In summary, the BSM critical surfaces of models A and C include regions for both $\left.\alpha_{\kappa}\right|_{M_{F}}$ and $\left.\alpha_{\kappa^{\prime}}\right|_{M_{F}}$ being perturbatively small. For even smaller values of $\left.\alpha_{\kappa}\right|_{M_{F}}$, larger values of $\left.\alpha_{\delta}\right|_{M_{F}}$ are required, and Higgs stability is not automatically guaranteed. The interplay of BSM input values on Planck-scale features is further detailed below (Sec. IV E).

We emphasize that our models are the first templates of asymptotically safe SM extensions with physical Higgs, top, and bottom masses, and which connect the relevant SM and BSM couplings at $\mathrm{TeV}$ energies with an interacting fixed point at highest energies. Another feature of our models is the low number $N_{F}$ of new fermion flavors required for this. In contrast, earlier attempts toward asymptotically safe SM extensions $[11,14,20,21]$ required moderate or large $N_{F}$, and either neglected the running of 

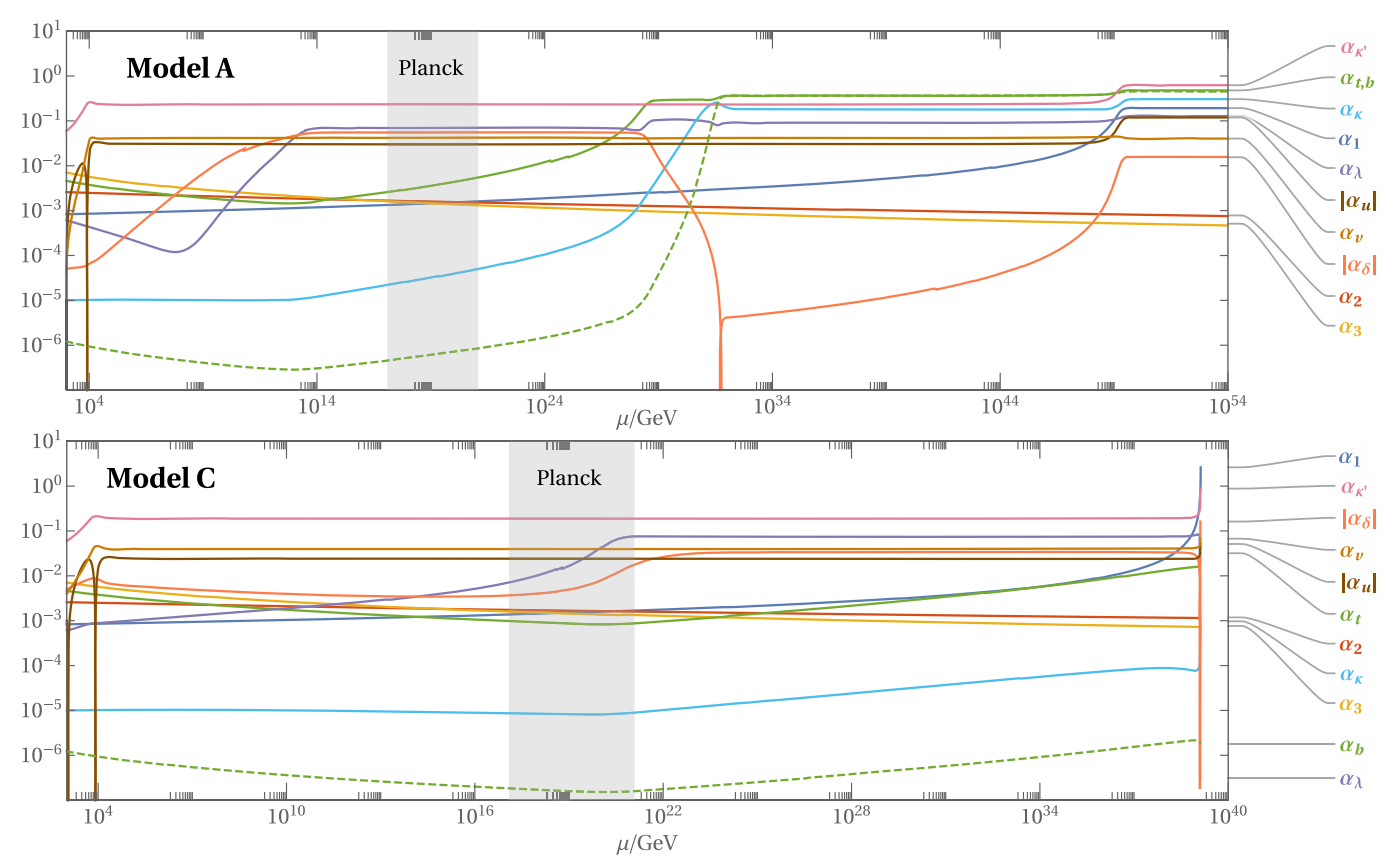

FIG. 12. As in Fig. 11 but for smaller $\alpha_{\kappa}$ (light blue line) and larger portal coupling $\left|\alpha_{\delta}\right|$ (orange line). The flow is stabilized by a crossover fixed point involving $\alpha_{\delta}$ (orange line). In model A, the flow continues into the same walking regime and UV fixed point as in Fig. 11, while model $\mathrm{C}$ runs into a pole way beyond the Planck scale. $\alpha_{u}$ (brown line) changes sign below $M_{\mathrm{Pl}}$.

quartic and portal couplings $[11,14]$ or used an unphysically large mass for the Higgs [21] in large- $N_{F}$ resummations which require further scrutiny [20,25]. It will therefore be interesting to test the fixed point at higherloop orders, once available, and nonperturbatively using lattice simulations [26], or functional renormalization.

\section{E. BSM critical surface}

We analyze the state of the vacuum at the Planck scale in dependence on the initial conditions of the BSM couplings at $M_{F}$ to determine the BSM critical surface in each model. In accord with the reasoning in Sec. IV D, the BSM Yukawas $\alpha_{y}$ and $\alpha_{\kappa}$ are varied at the matching scale, with the SM couplings fixed by (39). The remaining BSM couplings are, exemplarily, set to

$$
\left.\left\{\alpha_{\kappa^{\prime}}, \alpha_{\delta}, \alpha_{u}, \alpha_{v}\right\}\right|_{M_{F}}=\{0,5,1,4\} \times 10^{-5} .
$$

For each model, we then sample $141 \times 61$ different initial values $\left.\left(\alpha_{\kappa}, \alpha_{y}\right)\right|_{M_{F}}$ and integrate the RG flow at two-loop accuracy for all couplings from the matching scale to the Planck scale. The result for all models is shown in Fig. 13. Different parameter regions are color-coded to indicate the type of ground state at the Planck scale, or whether poles or instabilities arise prior to $M_{\mathrm{Pl}}$. Specifically, we distinguish regions in $\left.\alpha_{\kappa, y}\right|_{M_{F}}$ that yield stable vacua $V^{+}$(blue) or $V^{-}$ (green), according to (22), evaluated at the Planck scale. Regions with negative Higgs quartic are called metastable (yellow), if $0>\left.\alpha_{\lambda}\right|_{M_{\mathrm{Pl}}}>-10^{-4}$, and Higgs unstable if
$\left.\alpha_{\lambda}\right|_{M_{\mathrm{Pl}}}<-10^{-4}$. In the remaining regions with unstable vacuum (gray) either BSM quartics $\alpha_{u}, \alpha_{v}$ do not comply with (22) (regardless of $\alpha_{\lambda}$ and $\alpha_{\delta}$ ) or $\alpha_{u}, \alpha_{v}$, and $\alpha_{\lambda}$ do comply with (22), but $\alpha_{\delta}$ does not. Regions with Landau poles below or at the Planck scale are indicated in red.

Next, we discuss the pattern of results in Fig. 13. Connecting to the region of feeble couplings Fig. 7, Landau poles are present before the Planck scale within at least $\left.\alpha_{\kappa, y}\right|_{M_{F}} \lesssim 10^{-3}$ in models B, D, and F. For models $\mathrm{A}, \mathrm{C}$, and $\mathrm{E}$, on the other hand, within $\left.\alpha_{\kappa, y}\right|_{M_{F}} \lesssim 10^{-4}$ no poles arise and the Higgs potential is metastable or even becomes stable at the Planck scale (model C), just as depicted in Fig. 7.

Toward larger values of $\left.\alpha_{\kappa}\right|_{M_{F}}$, models $\mathrm{A}, \mathrm{C}$, and $\mathrm{E}$ exhibit a metastable and then unstable Higgs potential until $\left.\alpha_{\kappa}\right|_{M_{F}}$ is large enough to stabilize the potential as in Fig. 9. The vacuum configuration at $M_{\mathrm{Pl}}$ is then the same as at the matching scale, either $V^{+}$or $V^{-}$. For models B and F, $\left.\alpha_{\kappa}\right|_{M_{F}}>10^{-2}$ is required to move the Landau pole past the Planck scale, while this is not possible in model $\mathrm{D}$.

If we are increasing $\left.\alpha_{y}\right|_{M_{F}}$ instead, this leads eventually to the ground state $V^{+}$in the BSM potential, but Higgs stability is not guaranteed automatically; see Fig. 8. If not obstructed by poles, each model exhibits a narrow "belt" of parameters around $\left.\alpha_{\kappa^{\prime}}\right|_{M_{F}} \approx \mathcal{O}\left(10^{-3}\right)$ and any $\left.\alpha_{\kappa}\right|_{M_{F}}$, within which the BSM potential is unstable due to $\alpha_{u}<-\alpha_{v}$ in the $V^{-}$ground state. Here, Coleman-Weinberg resummations [12] or higher-order scalar self-interactions [13] 

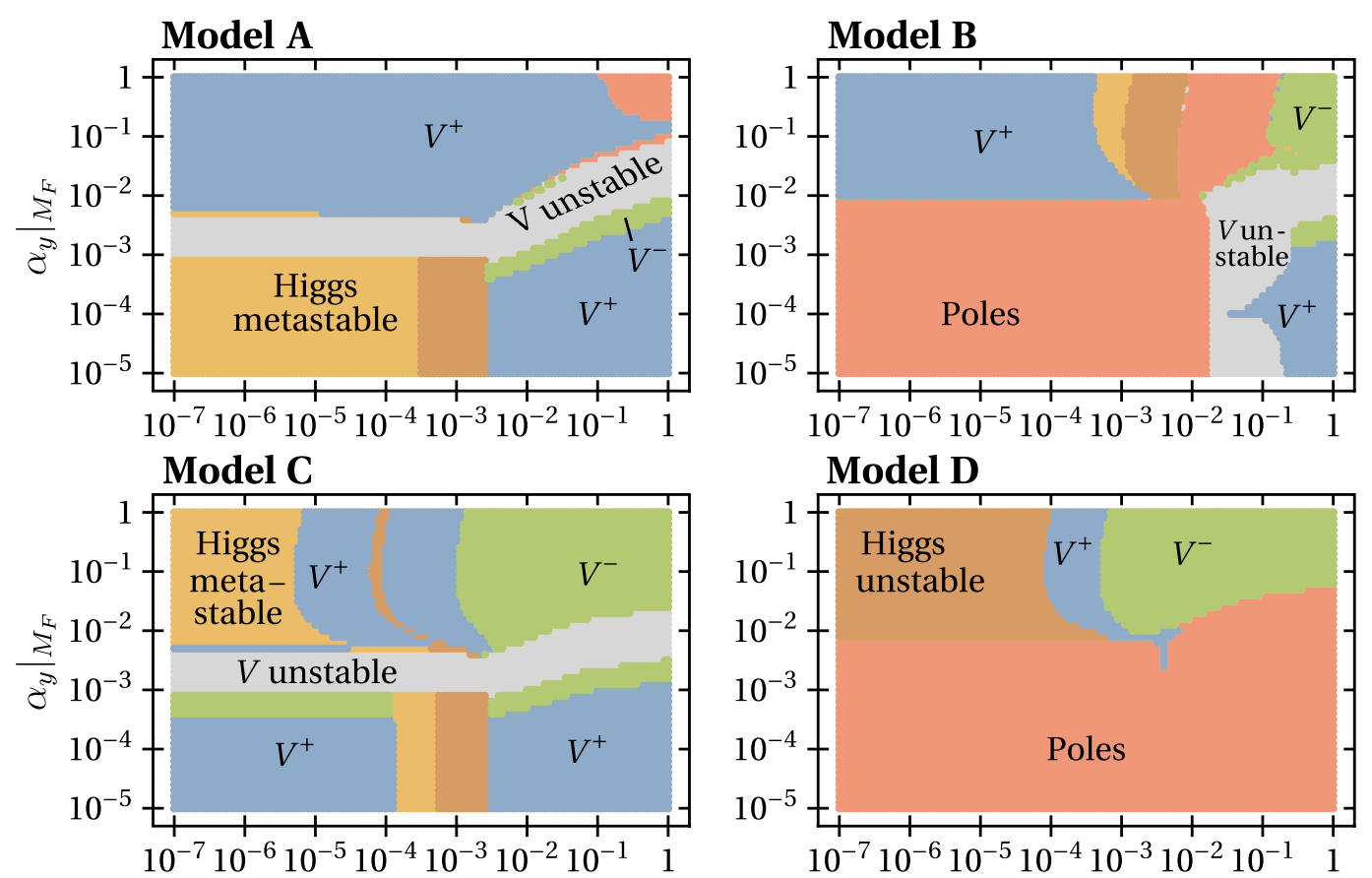

\section{Model E}

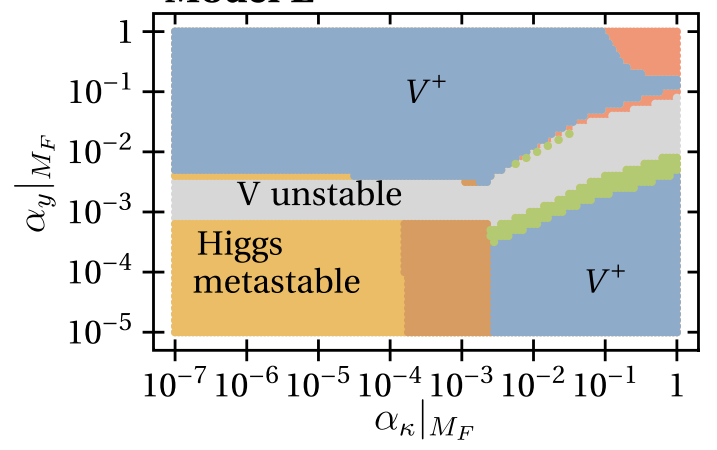

Model F

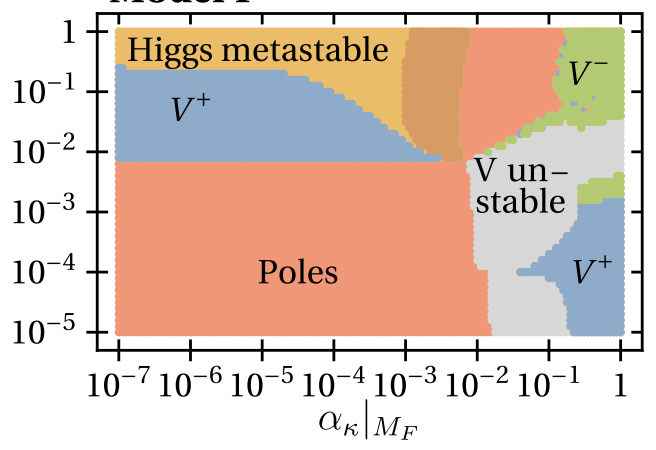

FIG. 13. BSM critical surface for models A-F with $\left.\left\{\alpha_{\kappa^{\prime}}, \alpha_{\delta}, \alpha_{u}, \alpha_{v}\right\}\right|_{M_{F}}=\{0,5,1,4\} \times 10^{-5}$ and values $\left.\left\{\alpha_{\kappa}, \alpha_{y}\right\}\right|_{M_{F}}$ at the matching scale. The colors indicate if the corresponding vacuum at the Planck scale $M_{\mathrm{Pl}}$ is either stable $V^{+}$(blue) or $V^{-}$(green), (22), an unstable BSM vacuum (gray), a stable vacuum for $\left.\alpha_{u, v}\right|_{M_{\mathrm{Pl}}}$ but with $\left.\alpha_{\lambda}\right|_{M_{\mathrm{Pl}}}<0$ (yellow for $\left.\alpha_{\lambda}\right|_{M_{\mathrm{Pl}}}>-10^{-4}$, otherwise brown), or if the RG flow runs into a pole (red). Resolution is $141 \times 61$ points per model.

should be included before definite conclusions about stability are taken.

Another feature of models $\mathrm{A}$ and $\mathrm{E}$ is that for $\left.\alpha_{\kappa, y}\right|_{M_{F}} \gtrsim$ $10^{-1}$ simultaneously, Landau poles occur before the Planck scale. For the other models, RG trajectories are stabilized around $M_{\mathrm{Pl}}$ in the $V^{-}$ground state by quartic interactions. However, this region is especially sensitive to corrections from higher-loop orders.

For models $\mathrm{A}$ and $\mathrm{C}$, the additional Yukawa interaction $\alpha_{\kappa^{\prime}}$ adds an extra dimension to the BSM critical surface. Its impact is further investigated in Fig. 14 (color-coding as in Fig. 13) where we exemplarily explore the vacuum state at the Planck scale within the $\left.\left(\alpha_{\kappa}, \alpha_{\kappa^{\prime}}\right)\right|_{M_{F}}$ parameter plane, and

$$
\left.\left\{\alpha_{y}, \alpha_{\delta}, \alpha_{u}, \alpha_{v}\right\}\right|_{M_{F}}=\{0,5,1,4\} \times 10^{-5}
$$

We find that the region with $\left.\alpha_{\kappa^{\prime}}\right|_{M_{F}} \lesssim 10^{-4}$ is very similar to the region $\left.\alpha_{y}\right|_{M_{F}} \lesssim 10^{-4}$ in Fig. 13, featuring a stable ground state for weakly coupled $\left.\alpha_{\kappa}\right|_{M_{F}}$. For both $\left.\alpha_{\kappa, k^{\prime}}\right|_{M_{F}} \gtrsim$ $10^{-2}$ the phenomena illustrated in Fig. 11 occur, implying a stable $V^{+}$region. The fate of the quadrant with $\left.\alpha_{\kappa^{\prime}}\right|_{M_{F}} \gtrsim$ $10^{-2}$ and $\left.\alpha_{\kappa}\right|_{M_{F}} \lesssim 10^{-2}$ hinges on the value of $\left.\alpha_{\delta}\right|_{M_{F}}$. As can be seen from Fig. 14, its flow can be stable, as in Fig. 12, while poles or Higgs metastability are possible as well.

The BSM critical surface at the matching scale of each model consists of the combined $V^{-}$plus $V^{+}$regions, with slices in the multidimensional parameter space shown in Figs. 13 and 14 in green and blue. All models A-F can be stable at least up to the Planck scale. The yellow (metastability) regions may be included as well, as this corresponds to the situation of the SM. In general, experimental constraints on the BSM critical surface apply for matching 


\section{Model A}

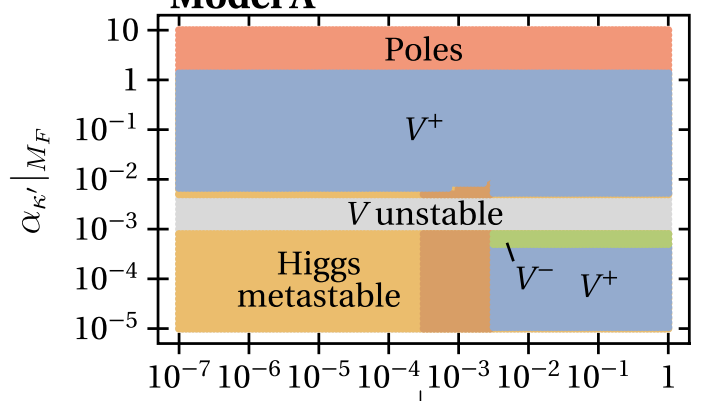

Model C $\left.\quad \alpha_{\kappa}\right|_{M_{F}}$

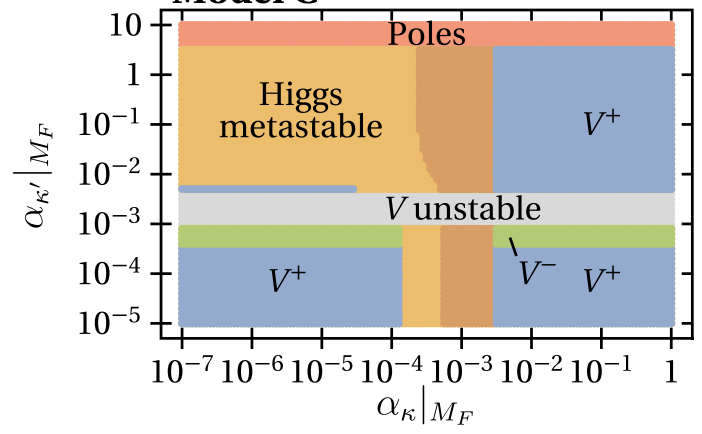

FIG. 14. As in Fig. 13 for models $\mathrm{A}$ and $\mathrm{C}$ in the $\left.\left\{\alpha_{\kappa}, \alpha_{\kappa^{\prime}}\right\}\right|_{M_{F}}$ plane with matching conditions $\left.\left\{\alpha_{y}, \alpha_{\delta}, \alpha_{u}, \alpha_{v}\right\}\right|_{M_{F}}=\{0,5,1,4\} \times$ $10^{-5}$. Resolution is $141 \times 61$ points per model.

scales around the $\mathrm{TeV}$ scale, a topic further discussed in the next Sec. V.

\section{PHENOMENOLOGY}

In this section, we investigate the phenomenological implications of our models. Specifically, in Sec. VA we discuss BSM sector production at hadron and lepton colliders, and in Sec. V B the decays of the BSM fermions and scalar. An important ingredient for phenomenology is mixing between SM and BSM fermions, the technical details for which are relegated to Appendix D. Resulting phenomenological consequences are worked out in Sec. VC and include dileptonic decays of the scalars. Constraints from Drell-Yan data on the matching scale are worked out in Sec. V D. Implications for the leptons' anomalous magnetic moments are studied in Sec. V E. In Sec. V F we show that the portal coupling $\delta$ in (21) together with $\kappa$ and $\kappa^{\prime}$ can provide a chirally enhanced contribution to the magnetic moments. This mechanism also induces EDMs for $C P$-violating couplings, discussed in Sec. V G. In Sec. V H we discuss constraints from charged LFV decays.

\section{A. BSM sector production}

Tree-level production channels of the BSM sector at $p p$ or $\ell \ell$ colliders are shown in Fig. 15. Since the fermions are colorless, pair production in $p p$ collisions is limited to quark-antiquark fusion to electroweak gauge bosons [diagrams 15(a) and 15(b)]. Single production through Yukawa interactions with $s$-channel Higgs [diagram 15(c)] is also possible. In $\ell \ell$ colliders, the $\psi$ can also be produced with $t$-channel Higgs or $S$ in pairs 15(d) and singly 15(e). The contribution to $\bar{\psi} \psi$ production from $s$-channel neutral bosons is especially relevant, since it is present in all models in study (except for model $\mathrm{E}$ ), in both $p p$ and $\ell \ell$ collisions, and all $N_{F}=3$ flavors of $\psi$ are produced. In the limit $M_{F} \gg m_{f}$, where $f$ is a quark or a lepton and $m_{f}\left(Q_{f}\right)$ denotes its mass (charge), the contribution to pair production via photon exchange at center of mass energy-squared $s$ reads

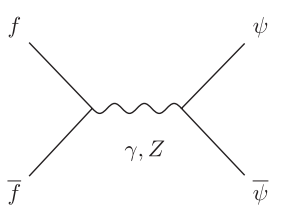

(a)

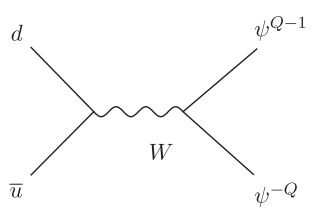

(b)

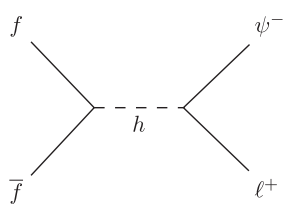

(c)

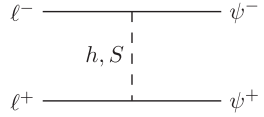

(d)

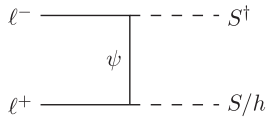

(f)

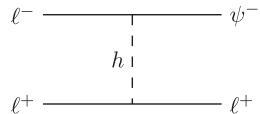

(e)

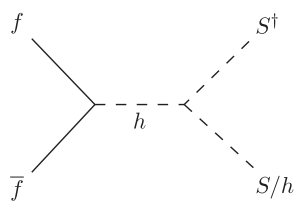

$(\mathrm{g})$

FIG. 15. Production channels of the BSM particles at $p p$ and $\ell \ell$ colliders, with $f=\ell, q$. In diagram (f) the $S$ and $S^{\dagger}$ labels are schematic for model A; see text for details. 


$$
\begin{aligned}
& \sigma_{\gamma}(\bar{f} f \rightarrow \bar{\psi} \psi) \\
& =N_{F} \frac{4 \pi}{3} \frac{\alpha_{e}^{2} Q_{f}^{2}}{s} \sum_{S U(2)_{L}} Q_{F}^{2} \sqrt{1-\frac{4 M_{F}^{2}}{s}}\left(1+\frac{2 M_{F}^{2}}{s}\right) \text { for } s>4 M_{F}^{2},
\end{aligned}
$$

where we summed over the $\psi$ 's flavors and $S U(2)_{L}$ components; $\alpha_{e}=e^{2} / 4 \pi$ denotes the fine structure constant. Corresponding cross sections are of the order $N_{F} Q_{f}^{2} \sum Q_{F}^{2} 90 \mathrm{fb} /(s[\mathrm{TeV}])$ [62]. Note the enhancement in models B and D which contain fermions with $\left|Q_{F}\right|=2$, and result in effective charge squares of $\sum Q_{F}^{2}=5$. The BSM scalars, which are SM singlets, can be pair produced at lepton colliders in models $\mathrm{A}$ and $\mathrm{C}$ through the Yukawa interactions $\left(\kappa^{\prime}\right)$ with $\psi$-exchange [diagram 15(f)]. The cross section, for $s>4 M_{S}^{2}$, then reads

$$
\begin{aligned}
& \sigma\left(\ell^{+} \ell^{-} \rightarrow S S^{\dagger}\right) \\
& =\frac{N_{F}}{32 \pi} \frac{\kappa^{\prime 4}}{s}\left(1-\frac{4 M_{S}^{2}}{s}\right)^{5 / 2} \int_{-1}^{1} \mathrm{~d} x x^{2}\left(1-x^{2}\right) \\
& \quad \times\left[\left(\frac{2\left(M_{F}^{2}-M_{S}^{2}\right)}{s}+1\right)^{2}-\left(1-\frac{4 M_{S}^{2}}{s}\right) x^{2}\right]^{-2} .
\end{aligned}
$$

Denote by $\operatorname{Re}[S]$ and $\operatorname{Im}[S]$ the real, $C P$-even, and $C P$-odd physical degrees of freedom of $S$, respectively. Together the Yukawas $\kappa$ and $\kappa^{\prime}$ induce single $S$ production, $\operatorname{Re}[S]$ or $\operatorname{Im}[S]$, in association with a Higgs [diagram 15(f)].

Another mechanism to probe the scalars is through $S$-Higgs mixing [diagram $15(\mathrm{~g})$ ], which arises if the portal coupling $\sim H^{\dagger} H \operatorname{Tr}\left[S^{\dagger} S\right]$ is switched on. In this diagram, the $h \operatorname{Re}[S] \operatorname{Re}[S]$ and $h \operatorname{Im}[S] \operatorname{Im}[S]$ couplings arise after electroweak symmetry breaking. In addition, the $h h \operatorname{Re}[S]$ vertex is possible when the scalar $S$ acquires a VEV. A detailed study of $\psi, S$ production at colliders is, however, beyond the scope of this work.

\section{B. BSM sector decay}

We discuss, in this order, the decays of the vectorlike leptons $\psi$ and the BSM scalar $S$. Both subsections contain a brief summary at the beginning.

\section{Fermions}

Depending on the representation, coupling, and mass hierarchies, the BSM fermions can decay through the Yukawa interactions to Higgs plus lepton or to $S$ plus lepton (only models A and C), while some members of the $S U(2)_{L}$-multiplets need to cascade down within the multiplet first through $W$-exchange. These are the states with electric charge $Q_{F}=-2$ (models B and D) and $Q_{F}=+1$ (model F). As detailed below, they allow for macroscopic lifetimes. Mixing with the SM leptons induces additional $\psi$-decays to $Z, W$ plus lepton which are discussed in Sec. V C.

The vectorlike fermions with $Q_{F}=0$ and $Q_{F}=-1$ can decay through the Yukawa interactions $(\kappa)$ to $h \nu$ and $h \ell^{-}$, respectively, except in model C, in which the Higgs couples to $S U(2)_{L}$-singlet leptons and only the $Q_{F}=-1$ decay takes place through $\kappa$. Neglecting the lepton mass, the decay rate into Higgs plus lepton is

$$
\Gamma(\psi \rightarrow \ell h)=\frac{\pi}{4} C_{\psi \ell}^{2} \alpha_{\kappa} M_{F}\left(1-\frac{m_{h}^{2}}{M_{F}^{2}}\right)^{2},
$$

where $C_{\psi \ell}=1 / \sqrt{2}$ for the $T_{3}=0$ states in models B, F, and $C_{\psi \ell}=1$ otherwise. For $\alpha_{\kappa} \gtrsim 10^{-14}$ and $M_{F}$ at least a tera electron volt, one obtains a lifetime $\Gamma^{-1} \lesssim \mathcal{O}\left(10^{-13}\right)$ s, which leads to a prompt decay. In models A (C), the decays $\psi_{i} \rightarrow \ell_{j} S_{j i}^{\dagger}\left(\psi_{i} \rightarrow \ell_{j} S_{j i}\right)$ are also allowed if the BSM scalars are lighter than the vectorlike fermions, with rate

$$
\Gamma(\psi \rightarrow \ell S)=\frac{\pi}{2} C_{\psi \ell}^{2} \alpha_{\kappa^{\prime}} M_{F}\left(1-\frac{M_{S}^{2}}{M_{F}^{2}}\right)^{2} .
$$

Models B and D contain $Q_{F}=-2$ fermions. After electroweak symmetry breaking, these cascade down through the weak interaction as $\psi^{-2} \rightarrow \psi^{-1} W^{*-}$, and subsequent decays.

The lifetime is then driven by the mass splitting within the multiplet. In the limit $M_{F} \gg m_{W}, m_{Z}$ one obtains for $\Delta m=M_{\psi^{-2}}-M_{\psi^{-1}}$ from SM gauge boson loops [63] $\Delta m \simeq \frac{\alpha_{2}^{\mathrm{PD}}}{2}\left(3 \sin \theta_{W}^{2} m_{Z}+k\right)$ and $k=m_{W}-m_{Z}(\operatorname{model} \mathrm{B})$, $k=0($ model $\mathrm{D})$, which is around a giga electron volt in both models. Corresponding decay rates $\Gamma\left(\psi^{-2} \rightarrow \psi^{-1} \ell \nu\right) \sim$ $G_{F}^{2} \Delta m^{5} /\left(15 \pi^{3}\right) \simeq 3 \times 10^{-13} \mathrm{GeV}(\Delta m /[\mathrm{GeV}])^{5}$ indicate around picosecond lifetimes of the $\psi^{-2}$, with a small, however macroscopic $c \tau \simeq 0.3 \mathrm{~mm}$ resulting in displaced vertex signatures that can be searched for at the LHC [64]. In model $\mathrm{F}$, the $Q_{F}=+1$ fermions decay similarly through $\psi^{+1} \rightarrow W^{+*} \psi^{0}$, with $\Delta m=M_{\psi^{+1}}-M_{\psi^{0}}=\alpha_{2}^{\mathrm{PDG}} M_{W} \sin ^{2} \frac{\theta_{W}}{2}$. Numerically, this is an order of magnitude smaller than the splitting in models B and D and suppresses the decay rate significantly further, allowing for striking long-lived charged particle signatures. Note that the presence of fermion mixing, discussed in the following, can induce more frequent decays unless couplings are very suppressed.

Note the upper limit on general mass splittings $\delta M$ within the fermion $S U(2)_{L}$-multiplets by the $\rho$-parameter [62]

$$
N_{F} S\left(R_{2}\right) \delta M^{2} \lesssim(40 \mathrm{GeV})^{2},
$$

where $S_{2}\left(R_{2}\right)$ is the Dynkin index of the representation $R_{2}$ of $S U(2)_{L}$ (see [11] for details). Specifically, $S_{2}=0,1 / 2,2$ for models $\mathrm{A}$ and $\mathrm{E}$, models $\mathrm{C}$ and $\mathrm{D}$, and models $\mathrm{B}$ and $\mathrm{F}$, 
respectively. The allowed splitting is hence about a few percent for tera-electron-volt-ish fermion masses.

\section{Scalars}

If kinematically allowed, the scalars $S_{i j}$ decay in all models through Yukawa couplings to $\psi \bar{\psi}$, and in models A and $\mathrm{C}$ to $\psi$ plus lepton. Only the flavor diagonal components can, except in the SM-singlet model E, in addition decay to electroweak gauge bosons through the $y$-Yukawa and a triangle loop with $\psi$ 's, $S \rightarrow G G^{\prime}$, with $G, G^{\prime}=$ $\gamma, W, Z$. Mixing of the vectorlike fermions with the SM leptons induces BSM scalar decays to dileptons, further discussed in Sec. V C.

For $M_{S}>M_{F}+m_{\ell}$ decays to vectorlike fermions and leptons through the mixed Yukawas $\left(\kappa^{\prime}\right)$, i.e., in models A and $\mathrm{C}$, are kinematically open. In all models, the decay to $\bar{\psi} \psi$ is possible for $M_{S}>2 M_{F}$ through the Yukawa coupling $y$. The tree-level decay rates for a given flavorspecific component $S_{i j}$ can be written as

$$
\begin{gathered}
\Gamma\left(S_{i j} \rightarrow \bar{\psi}_{i} l_{j}\right)+\Gamma\left(S_{i j} \rightarrow \bar{l}_{j} \psi_{i}\right)=2 \pi \alpha_{\kappa^{\prime}} M_{S}\left(1-\frac{M_{F}^{2}}{M_{S}^{2}}\right)^{2}, \\
\Gamma\left(S_{i j} \rightarrow \bar{\psi}_{i} \psi_{j}\right)+\Gamma\left(S_{i j} \rightarrow \overline{\psi_{j}} \psi_{i}\right)=2 \pi \alpha_{y} M_{S}\left(1-\frac{4 M_{F}^{2}}{M_{S}^{2}}\right)^{1 / 2+\xi},
\end{gathered}
$$

where model-dependent $S U(2)_{L}$ multiplicities in the final states are not spelled out explicitly. For instance, in model B, $S_{i j}$ decays to $\bar{\psi}_{i}^{-2} \psi_{j}^{-2}+\bar{\psi}_{i}^{-1} \psi_{j}^{-1}+\bar{\psi}_{i}^{0} \psi_{j}^{0}$ plus $C P$ conjugate ones. The loop-induced decays to gauge bosons read

$$
\Gamma\left(S_{i i} \rightarrow G G^{\prime}\right)=\frac{\alpha_{e}^{2} \alpha_{y}}{16 \pi} \frac{M_{S}^{3}}{M_{F}^{2}}\left|C_{G G^{\prime}} A_{1 / 2}(\tau)\right|^{2},
$$

where the coefficients $C_{G G^{\prime}}$ depend on the representation of $\psi$ and in the limit $M_{S} \gg M_{W}$ can be expressed as

$$
\begin{aligned}
C_{\gamma \gamma} & =S_{2}\left(R_{2}\right)+d\left(R_{2}\right) Y^{2}, \\
C_{Z Z} & =S_{2}\left(R_{2}\right) \tan ^{-2} \theta_{W}+d\left(R_{2}\right) Y^{2} \tan ^{2} \theta_{W}, \\
C_{W W} & =\frac{\sqrt{2}}{\cos ^{2} \theta_{W}} S_{2}\left(R_{2}\right), \\
C_{Z \gamma} & =\sqrt{2}\left(S_{2}\left(R_{2}\right) \tan ^{-1} \theta_{W}-d\left(R_{2}\right) Y^{2} \tan \theta_{W}\right) .
\end{aligned}
$$

In (58) and (59), $\xi=1$ and $\xi=0$ correspond to the scalar and pseudoscalar parts of $S$, respectively, and $A_{1 / 2}(\tau)=$ $\frac{2}{\tau^{2}}(\xi \tau+(\tau-\xi) f(\tau))$ with

$$
f(\tau)= \begin{cases}\arcsin [2](\sqrt{\tau}) & \text { for } \tau \leq 1 \\ -\frac{1}{4}\left(\ln \frac{1+\sqrt{1-\tau^{-1}}}{1-\sqrt{1-\tau^{-1}}}-i \pi\right)^{2} & \text { for } \tau>1\end{cases}
$$

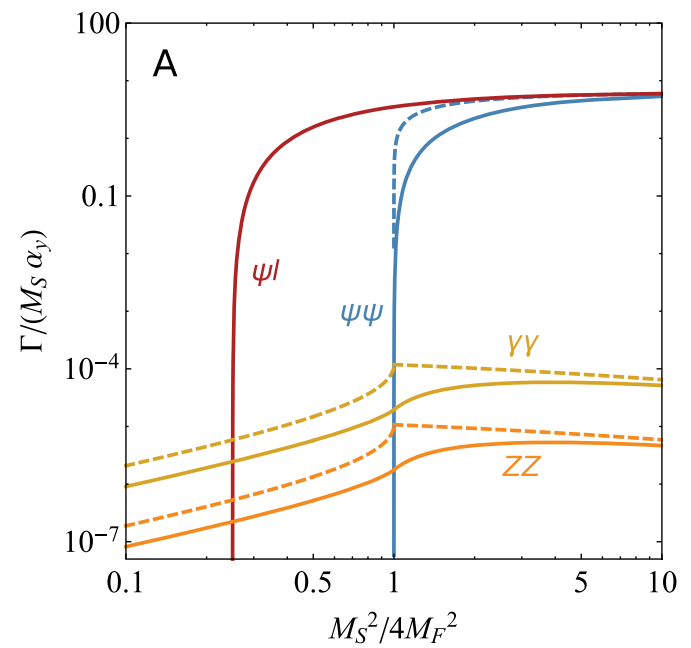

FIG. 16. Reduced decay rates $\Gamma /\left(M_{S} \alpha_{y}\right)$ of the flavor-specific components of the BSM scalar $S$ (58), (59) in model A for $\alpha_{y}=\alpha_{\kappa^{\prime}}$. Full (dashed) lines correspond to the scalar (pseudoscalar) decays; for $S \rightarrow \psi \ell$ they coincide. The decay rate into $Z \gamma$ lies between the $Z Z$ and $\gamma \gamma$ curves.

and $\tau=M_{S}^{2} / 4 M_{F}^{2}$ [65]. In the case of one of the $S_{i j}$ mixing with angle $\beta$ with the Higgs, the real part of $S_{i j}$ can decay through mixing with rate $\Gamma_{\text {mix }}=\sin ^{2} \beta \Gamma_{h}^{\mathrm{SM}}$, where $\Gamma_{h}^{\mathrm{SM}}$ is the decay rate of the Higgs in the SM.

In model A the main $S$ decay channels are $\bar{\psi} \psi$ and $\psi \ell$, followed by the decay to photons. Other gauge boson modes are further suppressed, as for $T_{3}=0$ holds $1>\left(C_{Z \gamma}\right)^{2}=2 \tan \theta_{W}^{2}>\left(C_{Z Z}\right)^{2}=\tan \theta_{W}^{4}$. The reduced rates $\Gamma /\left(M_{S} \alpha_{y}\right)$ as a function of $\tau$ for model A are shown in Fig. 16 for $\alpha_{y}=\alpha_{\kappa^{\prime}}$.

In models $\mathrm{B}, \mathrm{C}, \mathrm{D}$, and $\mathrm{F}$ the vectorlike fermions are charged under $S U(2)_{L}$ and allow for decays to $W^{+} W^{-}$. When kinematically allowed, the tree-level decays into $\bar{\psi} \psi$ are dominant. For model B this is shown in Fig. 17. The hierarchy between the gauge boson decay rates in model B reads $\Gamma_{Z Z}^{\mathrm{B}}>\Gamma_{\gamma \gamma}^{\mathrm{B}}>\Gamma_{W W}^{\mathrm{B}}>\Gamma_{Z \gamma}^{\mathrm{B}}$, and in model $\mathrm{C}$ $\Gamma_{Z Z}^{\mathrm{C}}>\Gamma_{\gamma \gamma}^{\mathrm{C}}>\Gamma_{Z \gamma}^{\mathrm{C}} \approx \Gamma_{W W}^{\mathrm{C}}$. In model $\mathrm{D}$, the $S_{i i} \rightarrow G G^{\prime}$ hierarchies are $\Gamma_{\gamma \gamma}^{\mathrm{D}}>\Gamma_{Z Z}^{\mathrm{D}}>\Gamma_{Z \gamma}^{\mathrm{D}}>\Gamma_{W W}^{\mathrm{D}}$, whereas in model $\mathrm{F}$ $\Gamma_{Z Z}^{\mathrm{F}}>\Gamma_{Z \gamma}^{\mathrm{F}}>\Gamma_{W W}^{\mathrm{F}}>\Gamma_{\gamma \gamma}^{\mathrm{F}}$.

For $M_{S}<M_{F}$ and negligible $\alpha_{y}$ one may wonder whether $S$ can decay at all. However, fermion mixing induces decays to SM leptons or neutrinos, discussed next.

\section{Fermion mixing}

Mixing between SM leptons and BSM fermions provides relevant phenomenology. Mixing angles-in the small angle approximation to make the parametric dependence explicit-for the left-handed $\left(\theta_{L}^{M}\right)$ and right-handed $\left(\theta_{R}^{M}\right)$ fermions, with the model $\mathrm{M}$ indicated as superscript, are given in Table VI. Details are given in Appendix D. We discuss, in this order, the impact of mixing on scalar 


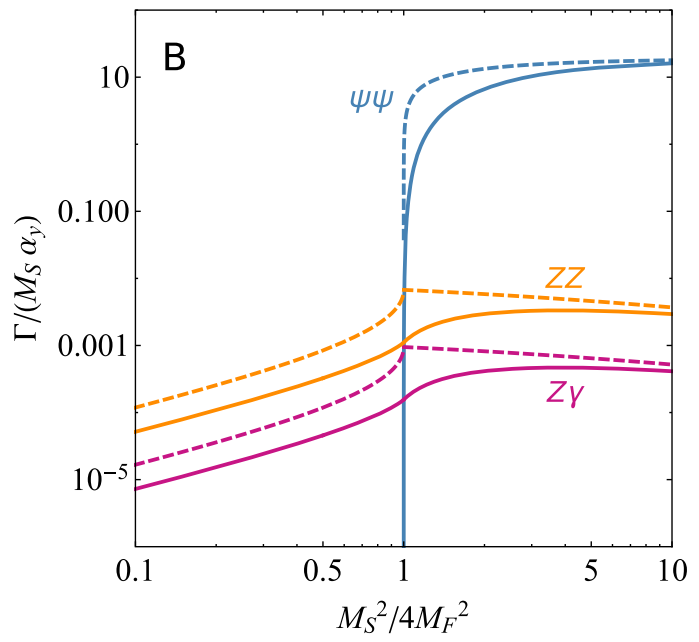

FIG. 17. Reduced decay rates $\Gamma /\left(M_{S} \alpha_{y}\right)$ of the flavor-specific components of the BSM scalar $S$ (58) and (59) in model B. Full (dashed) lines correspond to the scalar (pseudoscalar) decays. The decay rates in model $\mathrm{B}$ into $W W$ and $\gamma \gamma$ satisfy $\Gamma_{Z Z}^{\mathrm{B}}>\Gamma_{\gamma \gamma}^{\mathrm{B}}>\Gamma_{W W}^{\mathrm{B}}>\Gamma_{Z \gamma}^{\mathrm{B}}$.

decays, modified electroweak and Higgs couplings and decays of vectorlike leptons to $Z, W+\mathrm{SM}$ lepton. The results are important for experimental searches because they imply that all $S_{i j}$ and $\psi_{i}$ eventually decay to SM leptons, charged ones, and neutrinos, with the only exceptions being the diagonal $S_{i i} \rightarrow G G^{\prime}$ decays.

\section{LFV-like scalar decay}

In models $\mathrm{A}$ and $\mathrm{C}$, mixing induces tree-level decays $S_{i j} \rightarrow \ell_{i}^{ \pm} \ell_{j}^{\mp}$ at the order $\kappa^{\prime} \theta_{L}^{A}, \kappa^{\prime} \theta_{R}^{C} \simeq \kappa^{\prime} \frac{\kappa v_{h}}{\sqrt{2} M_{F}}$, using the angles of Table VI. These can be competitive with decays to electroweak bosons: for instance, taking $\kappa^{\prime} \sim y$ and for $\theta_{L}^{A}$ of order $10^{-3}$ or larger they dominate over $S \rightarrow \gamma \gamma$ in model A. Unless the mixing is strongly suppressed,

TABLE VI. Mixing angles of the $Q_{F}=-1$ fermions $\left(\theta^{M}\right)$ and the $Q_{F}=0$ fermions $\left(\theta^{0, M}\right)$ (see Table I), with $m_{2}=M_{F}+y v_{s} / \sqrt{2}$. In $V^{-}$, the direction that is aligned with the vacuum (second generation) presents different mixing angles. We have $\theta_{L, R}^{C}=\theta_{R, L}^{A}$ for model $\mathrm{C}$ and $\theta_{L, R}^{D}=\sqrt{2} \theta_{R, L}^{B}$ for model D. We also find $\theta_{L}^{0, E}=\sqrt{2} \theta_{L}^{0, F}=\theta_{L}^{0, B}$ and $\theta_{L, R}^{F}=\sqrt{2} \theta_{L, R}^{B}$ in models $\mathrm{E}$ and $\mathrm{F}$. The additional factor of $1 / \sqrt{2}$ in $\theta^{B}$ and $\theta^{0, F}$ originates from Clebsch-Gordan coefficients (31); see Appendix D for details.

\begin{tabular}{lcccccc}
\hline \hline Vacuum & $\theta_{L}^{A}$ & $\theta_{R}^{A}$ & $\theta_{L}^{B}$ & $\theta_{R}^{B}$ & $\theta_{L}^{0, B}$ & $\theta_{L}^{0, C}$ \\
\hline $\boldsymbol{V}^{+}$ & $\frac{\kappa v_{h}}{\sqrt{2} m_{2}}$ & $\frac{\kappa^{\prime} v_{s}}{\sqrt{2} m_{2}}$ & $\frac{\kappa v_{h}}{2 m_{2}}$ & $\frac{\kappa v_{h}^{2} y_{\ell}}{2 \sqrt{2} M_{2}^{2}}$ & $\frac{\kappa v_{h}}{\sqrt{2} m_{2}}$ & $\frac{\kappa^{\prime} v_{s}}{\sqrt{2} m_{2}}$ \\
$\boldsymbol{V}^{-}\left(\boldsymbol{\psi}_{\mathbf{2}}-\ell_{\mathbf{2}}\right)$ & $\frac{\kappa v_{h}}{\sqrt{2} M_{F}}$ & $\frac{\kappa^{\prime} v_{s}}{\sqrt{2} m_{2}}$ & $\frac{\kappa v_{h}}{2 M_{F}}$ & $\frac{\kappa v_{h}^{2} y_{\ell}}{2 \sqrt{2} M_{F}^{2}}$ & $\frac{\kappa v_{h}}{\sqrt{2} M_{F}}$ & $\frac{\kappa^{\prime} v_{s}}{\sqrt{2} m_{2}}$ \\
$\boldsymbol{V}^{-}\left(\boldsymbol{\psi}_{\mathbf{1}, 3}-\ell_{\mathbf{1 , 3}}\right)$ & $\frac{\kappa v_{h}}{\sqrt{2} M_{F}}$ & $\frac{\kappa v_{h}^{2} y_{\ell}}{2 M_{F}^{2}}$ & $\frac{\kappa v_{h}}{2 M_{F}}$ & $\frac{\kappa v_{h}^{2} y_{\ell}}{2 \sqrt{2} M_{F}^{2}}$ & $\frac{\kappa v_{h}}{\sqrt{2} M_{F}}$ & 0 \\
\hline \hline
\end{tabular}

$\kappa^{\prime} \theta_{L}^{A}, \kappa^{\prime} \theta_{R}^{C} \lesssim 10^{-7}$ for $M_{S}$ at the tera-electron-volt scale, the $S$ lifetime is below picoseconds and is too short for a macroscopic decay length.

In models $\mathrm{B}, \mathrm{D}$, and $\mathrm{F}$, for $M_{S}>M_{F}$, fermion mixing induces the decays $S_{i j} \rightarrow \psi_{j} \ell_{i}$ (models B and F) and $S_{i j} \rightarrow$ $\psi_{i} \ell_{j}$ (model D) at the order $y \frac{\kappa v_{h}}{\sqrt{2} M_{F}}$. For $M_{S}<M_{F}$, the decays $S_{i j} \rightarrow \ell_{i}^{ \pm} \ell_{j}^{\mp}$ at the order $y \theta_{L}^{M} \theta_{R}^{M} \simeq y\left(\frac{\kappa v_{h}}{\sqrt{2} M_{F}}\right)^{2} \frac{y_{\ell} v_{h}}{\sqrt{2} M_{F}}$ are the leading ones. Using (58) again, one obtains a lifetime of picoseconds or above for a suppression factor $y \theta_{L}^{M} \theta_{R}^{M} \lesssim 10^{-7}$. Because of its flavor dependence, the suppression of the mixing is stronger for tau-less final states. This could allow for displaced decays into dielectrons, dimuons, and $e^{ \pm} \mu^{\mp}$, while at the same time, those into ditaus, $e^{ \pm} \tau^{\mp}$, and $\mu^{ \pm} \tau^{\mp}$ could remain prompt.

Last, for models with $Q_{F}=0$ fermion decays $S \rightarrow \bar{\psi}_{j}^{0} \nu_{i}$ are also allowed for $M_{S}>M_{F}$, occurring at order $y \theta_{L}^{0, M}$ for models $\mathrm{B}, \mathrm{E}$, and $\mathrm{F}$ and at order $\kappa^{\prime}$ for model $\mathrm{C}$. In the case of model E, this is the only available decay mode of the $S_{i j}$ (apart from $S \rightarrow \bar{\psi} \psi$ if allowed), leading to below-picosecond lifetimes for $y \theta_{L}^{0, E} \simeq y \kappa v_{h} / \sqrt{2} M_{F} \lesssim 10^{-7}$. Study of the different $S$ decay modes into various gauge bosons or fermions can be used for experimental discrimination of models. The patterns of final state leptons in LFV-like ${ }^{2}$ decays, $e, \mu$, or $\tau$ can help to understand hierarchies.

\section{Impact on $Z, W$, and Higgs couplings}

Fermion mixing gives rise to tree-level effects in the couplings of leptons and vectorlike fermions to the massive electroweak bosons. In the case of the $Z$ couplings to two leptons, the Lagrangian in the fermion mass basis $\mathcal{L}_{Z}=\frac{g_{2}}{2 \cos \theta_{w}}\left[\bar{\ell} \gamma^{\mu}\left(g_{V}^{\ell}-\gamma^{5} g_{A}^{\ell}\right) \ell+g^{\nu} \bar{\nu} \gamma^{\mu}\left(1-\gamma^{5}\right) \nu\right] Z_{\mu}$ acquires couplings

$$
g_{\substack{\ell \\ \ell}}^{\ell}=g_{V}^{\ell, \mathrm{SM}}+s_{\theta_{L}}^{2}\left(T_{\psi^{-1}}^{3}+1 / 2\right) \pm s_{\theta_{R}}^{2} T_{\psi^{-1}}^{3},
$$

with respect to their SM values $g_{V}^{\ell}=-1 / 2+2 s_{w}^{2}$ and $g_{A}^{\ell}=-1 / 2$, and where $T_{\psi^{-1}}^{3}$ is the isospin of the $Q_{F}=-1$ component of the vectorlike fermions in each model. The rotation angles are to be taken from Table VI according to the chosen vacuum structure and the lepton flavor $\ell$. In the case of model A (C), one finds $T_{\psi^{-1}}^{3}=0\left(T_{\psi^{-1}}^{3}=-1 / 2\right)$, yielding modifications purely proportional to $s_{\theta_{L}}^{2}\left(s_{\theta_{R}}^{2}\right)$. In models $\mathrm{B}, \mathrm{E}$, and $\mathrm{F}$ one finds $\theta_{R} \ll \theta_{L}$, while model $\mathrm{D}$ presents $\theta_{L} \ll \theta_{R}$, so that in all models the $g^{\ell}$ present modifications proportional to $\kappa v_{h} / M_{F}$. In models with $Q_{F}=0$ fermions (B, C, E, and F), the $Z$ couplings to two neutrinos become

\footnotetext{
${ }^{2}$ Despite the different lepton flavors in the final state processes such as $S_{i j} \rightarrow \ell_{i}^{ \pm} \ell_{j}^{\mp}$ are, strictly speaking, LFV-like only because flavor is conserved in the decay.
} 


$$
g^{\nu}=g^{\nu, \mathrm{SM}}+\Delta g^{\nu}=g^{\nu, \mathrm{SM}}+s_{\theta_{L}^{0}}^{2}\left[T_{\psi^{0}}^{3}-1 / 2\right]
$$

with $g^{\nu, \mathrm{SM}}=1 / 2$. In model C, for which $T_{\psi^{0}}^{3}=1 / 2, g^{\nu}$ remains unaffected. Therefore, in all models $Z$ data mainly constrains the mixing angles proportional to $\kappa v_{h} / M_{F}$. Measurements of the $Z$ couplings to charged leptons and the electron-flavored neutrinos demand $\Delta g \lesssim 10^{-3}$ or smaller [53], which implies

$$
\alpha_{\kappa} \lesssim 4 \times 10^{-4}\left(M_{F} / \mathrm{TeV}\right)^{2} .
$$

Modifications of the $W$ couplings remain also in agreement with $W$ decay measurements if (64) is fulfilled (see Appendix E for details). Additionally, Higgs couplings are modified by mixing as well. Since charged leptons acquire mass from several Yukawa interactions, the couplings of $\mathcal{L}_{h \ell \ell}=\frac{y_{\ell}}{\sqrt{2}} \bar{\ell} \ell h$ in the mass basis fulfill

$y_{\ell}=y_{\ell}^{\mathrm{SM}}+\sin \theta_{L}^{\ell}\left(\kappa^{\prime} \frac{v_{s}}{v_{h}} \cos \theta_{R}^{\ell}-\sqrt{2} \frac{M_{F}}{v_{h}} \sin \theta_{R}^{\ell}\right)$

for model A, while replacing $L \leftrightarrow R$ gives the expression for model C. In all other models, the $\kappa^{\prime}$ term is absent. For angles fulfilling $Z$ vertex constraints according to Eq. (64), Higgs signal strength bounds are avoidable for all leptons $[53,66]$.

\section{Electroweak decays of vectorlike leptons}

Finally, mixing induces decays of the vectorlike fermions to weak bosons and leptons at tree level, with rates

$$
\begin{aligned}
& \Gamma\left(\psi_{i}^{Q} \rightarrow Z f_{i}^{Q}\right) \\
& \quad=\frac{M_{F}}{64 \pi} \frac{g_{2}^{2}}{\cos ^{2} \theta_{w}}\left(g_{V}^{2}+g_{A}^{2}\right)\left(1-r_{Z}\right)^{2}\left(2+1 / r_{Z}\right), \\
& \Gamma\left(\psi_{i}^{Q} \rightarrow W^{-} f_{i}^{Q+1}\right) \\
& \quad=\frac{M_{F}}{64 \pi} g_{2}^{2}\left[\left(c_{L}^{W}\right)^{2}+\left(c_{R}^{W}\right)^{2}\right]\left(1-r_{W}\right)^{2}\left(2+1 / r_{W}\right),
\end{aligned}
$$

where $r_{i}=M_{i}^{2} / M_{F}^{2}, f^{-1}=\ell, f^{0}=\nu$, and the coefficients $c_{L, R}^{W}$ and $g_{V, A}$ are collected in Tables X and XI, respectively, for all models. Let us discuss the decays of the chargeless $\psi^{0}$ in model $\mathrm{C}$, which occur exclusively through its mixing unless $\psi^{0} \rightarrow S \nu$ via $\kappa^{\prime}$ is allowed. For the universal vacuum $V^{+}$and for the flavor in which the flavor-specific vacuum $V^{-}$points, it is important to note that the $\psi^{0}$ is lighter than the $\psi^{-1}$ by $\Delta m_{C}=M_{\psi^{-1}}-M_{\psi^{0}}=$ $\alpha_{2}^{\mathrm{PDG}} \sin \theta_{W}^{2} m_{Z} / 2 \simeq 0.4 \mathrm{GeV}$. This difference causes isospin breaking in the mixing angles given in Table VI, which induces a Cabibbo-Kobayashi-Maskawa-like misalignment between up $T_{3}=1 / 2$ and down $T_{3}=-1 / 2$ sectors $\theta_{L}^{0, C}-\theta_{L}^{C} \simeq \theta_{L}^{C}\left(\Delta m_{C} / M_{F}\right)$, such that the decay $\psi^{0} \rightarrow$ $\ell^{\prime-} W^{+*} \rightarrow \ell^{\prime-} \ell^{+} \nu$ can take place. Assuming $\theta_{R}^{C} \ll \theta_{L}^{C}$,

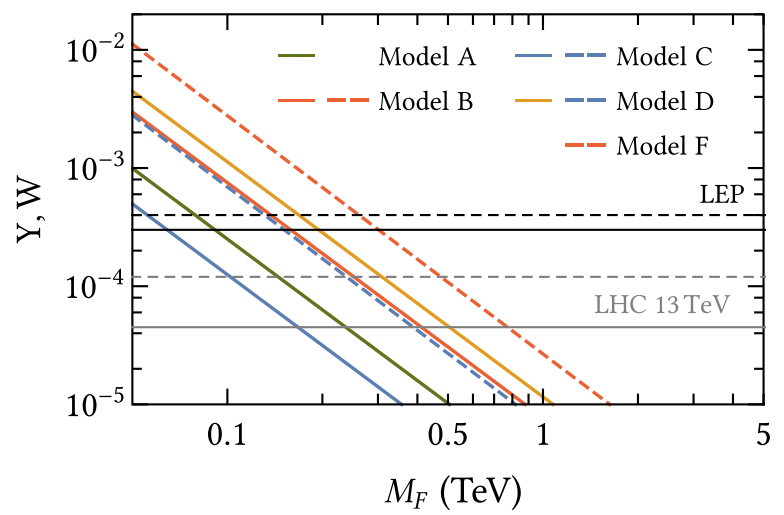

FIG. 18. The electroweak parameters $Y$ (full lines) and $W$ (dashed lines) for models $\mathrm{A}-\mathrm{D}$ and $\mathrm{F}$ as functions of the BSM fermion mass, and in comparison with the most stringent constraints from either LHC $8 \mathrm{TeV}$ or the Large Electron and Positron Collider (LEP, black lines), and the projected sensitivity of LHC $13 \mathrm{TeV}$ (gray) taken from [68]. $W$ constraints in models $\mathrm{B}, \mathrm{F}$ (orange dashed line) and C, D (blue dashed line) are identical.

we estimate $\Gamma\left(\psi^{0} \rightarrow \ell^{\prime-} \ell^{+} \nu\right) \sim G_{F}^{2}\left|\theta_{L}^{C}\right|^{2} \Delta m_{C}^{2} M_{F}^{3} /\left(192 \pi^{3}\right) \simeq$ $4 \times 10^{-6} \mathrm{GeV}\left|\theta_{L}^{C}\right|^{2}\left(M_{F} /[\mathrm{TeV}]\right)^{3}$. Unless $\theta_{L}^{C} \lesssim 10^{-3}$, the $\psi^{0}$ decays faster than picoseconds.

For the flavors $k$ in the lepton-specific vacuum $V^{-}$which do not get a corresponding VEV in $S$, the left- and righthanded angles have the opposite hierarchy, fulfilling $\theta_{L}^{C} \ll \theta_{R}^{C}$. Since $\theta_{R}^{0, C}=0$, the $\psi_{k}^{0}$ decay promptly through $\psi_{k}^{0} \rightarrow W^{-} \ell_{k}^{+}$with $\left|c_{R}^{W}\right|=\sin \theta_{R}^{C} \simeq \kappa v_{h} / \sqrt{2} M_{F}$.

\section{Drell-Yan}

Modifications of the running of the electroweak couplings can be constrained directly from charged and neutral current Drell-Yan processes. Of particular interest are the electroweak precision parameters $W$ and $Y$, which are linearly dependent on the BSM contribution to the running of $\alpha_{2}$ and $\alpha_{1}$, respectively, as [67]

$$
W, Y=\alpha_{2,1} \frac{C_{2,1}}{10} \frac{M_{W}^{2}}{M_{F}^{2}}\left(B_{2,1}^{\mathrm{SM}}-B_{2,1}\right),
$$

where $C_{2}=1$ and $C_{1}=3 / 5$. A lower limit on the mass of the vectorlike fermions can be directly extracted from experimental bounds on $W, Y$ [68]. As shown in Fig. 18, these require $M_{F} \gtrsim 0.1 \mathrm{TeV}$ for model $\mathrm{A}$ and $M_{F} \gtrsim 0.3$ and $0.2 \mathrm{TeV}$ for models B and $\mathrm{C}$, respectively. In models $\mathrm{D}$ and $\mathrm{F}$ one obtains $M_{F} \gtrsim 0.2$ and 0.3 , respectively, while in model $\mathrm{E}$ one cannot extract bounds due to the BSM sector being uncharged under the SM gauge symmetries. The bound for model B excludes fixed points $B_{2}$ and $B_{4}$, which can only be matched at $M_{F} \simeq 0.02 \mathrm{TeV}$. Remarkably, the fixed points that remain viable in terms of matching are only those which present a free $\alpha_{2}$. The effect of two-loop corrections in $W, Y$ may be estimated by taking the effective coefficients $B_{i}^{\text {eff }}$ instead of $B_{2,1}$ in (67). In our 
matching scenarios, this typically induces relative changes of order $1 \%$ or less in $W, Y$ with respect to the one-loop values, and $W, Y$ remain positive. The smallness of these corrections is due to the fact that all couplings at low scales present values of order $10^{-2}-10^{-3}$, which are suppressing the two-loop effects, while $B_{2,1}$ are typically of order 1 or larger.

\section{E. Anomalous magnetic moments}

The measurements of the electron and muon anomalous magnetic moments are in tension with SM predictions, offering hints for new physics. In the case of the muon, the long-standing discrepancy amounts to [53]

$$
\Delta a_{\mu}=a_{\mu}^{\exp }-a_{\mu}^{\mathrm{SM}}=268(63)(43) \times 10^{-11} .
$$

Adding uncertainties in quadrature, this represents a $3.5 \sigma$ deviation from the SM, while recent theory predictions find up to $4.1 \sigma[69,70] .{ }^{3}$ For the magnetic moment of the electron, recent measurements lead to

$$
\Delta a_{e}=a_{e}^{\mathrm{exp}}-a_{e}^{\mathrm{SM}}=-88(28)(23) \times 10^{-14},
$$

corresponding to a pull of $-2.4 \sigma$ from the SM prediction [75,76]. For earlier BSM considerations, see Ref. [77].

From a model building perspective it is important to understand which new physics ingredients are required to explain the anomalies (68) and (69) simultaneously. Given that the electron and muon deviations point in opposite directions, it is commonly assumed that an explanation requires the manifest breaking of lepton flavor universality. BSM models which explain both anomalies by giving up on lepton flavor universality have used new light scalar fields [78-83], supersymmetry [84-87], bottom-up models [88,89], leptoquarks [90,91], two-Higgs doublet models $[92,93]$, or other BSM mechanisms which treat electrons and muons manifestly differently [94-102]. In the spirit of Occam's razor, however, we have shown recently that the data can very well be explained without any manifest breaking of lepton universality [40], which is in marked contrast to any of the alternative explanations offered by [78-101].

In this and the following subsection, we detail how the models $\mathrm{A}, \mathrm{B}, \mathrm{C}, \mathrm{D}$, and $\mathrm{F}$ induce anomalous magnetic moments at one-loop, and why, ultimately, only models A and $\mathrm{C}$ can explain the present data. Note that model $\mathrm{E}$ does not appear in the list, the reason being that the charged SM leptons do no longer couple to BSM fermions after electroweak symmetry breaking. The setting previously

\footnotetext{
${ }^{3}$ The possibility of rendering $\Delta a_{\mu}$ insignificant has recently been suggested by a lattice determination of the hadronic vacuum polarization [71]. Further scrutiny is required [72] due to tensions with electroweak data $[73,74]$ and earlier lattice studies.
}

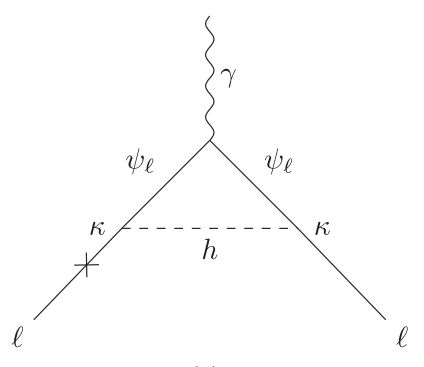

(a) (b)

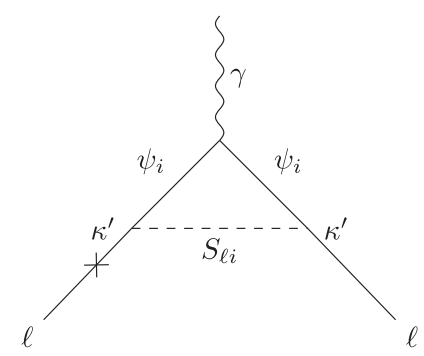

FIG. 19. Contributions to $\Delta a_{\ell}(\ell=e, \mu, \tau)$ with a lepton chiral flip (cross on solid line) via $h$ (a) or $S_{i \ell}$ exchange, with $i=1,2$, 3 , only present in models A and C (b).

put forward by us in [40] corresponds to model A and model $\mathrm{C}$ of the present paper.

Specifically, new physics contributions to $\Delta a_{\ell}$ arise through the one-loop diagrams shown in Fig. 19. In the limit where $M_{F}$ is much larger than the mass of the lepton and the scalar propagating in the loop, the new physics (NP) contribution typically scales as

$$
a_{\ell}^{\mathrm{NP}} \sim \alpha_{\eta} \frac{m_{\ell}^{2}}{M_{F}^{2}},
$$

where $m_{\ell}$ denotes the lepton mass and $\eta=\kappa, \kappa^{\prime}$ is one of the mixed Yukawa couplings; see Appendix B for details. For couplings $\kappa^{\prime}, \kappa$ of comparable order, the largest contribution comes from the latter, which couples the vectorlike fermions to the lighter scalar (the Higgs). The parameter space $\alpha_{\kappa}, M_{F}$ compatible with (68) is shown in Fig. 20. As obvious from (B2), Eq. (70) is manifestly positive, and cannot account for $\Delta a_{e}$. For the muon anomaly (68), the coupling $\alpha_{\kappa} M_{F}^{-2} \approx(1.4 \pm 0.4) \mathrm{TeV}^{-2}$ in models $\mathrm{A}, \mathrm{C}$, and $\mathrm{D}$ as well as $\alpha_{\kappa} M_{F}^{-2} \approx$ $(4.2 \pm 1.2) \mathrm{TeV}^{-2}$ for models $\mathrm{B}$ and $\mathrm{F}$ is required. This is, however, ruled out by the constraint (64). We learn that

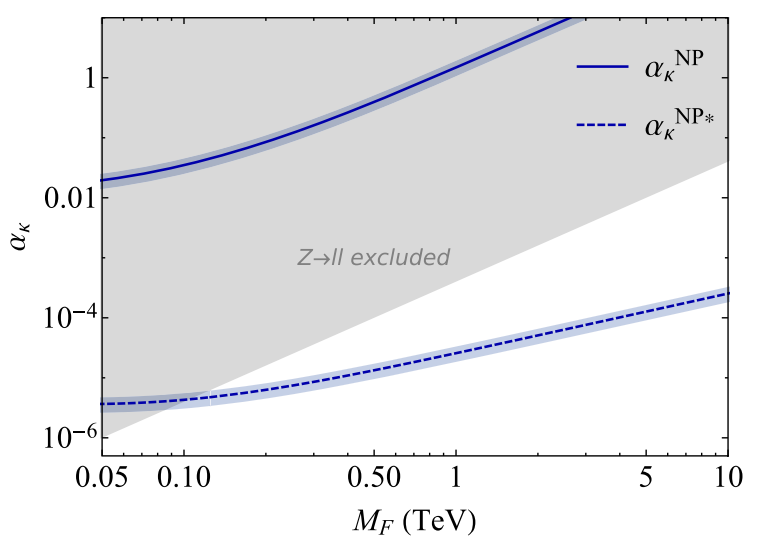

FIG. 20. Requisite values of $\alpha_{\kappa}$ to account for $\Delta a_{\mu}$ (68) for new physics contributions scaling as (70) (full line) and (71) (dotted line). The shaded region is excluded by $Z$-data (64). 


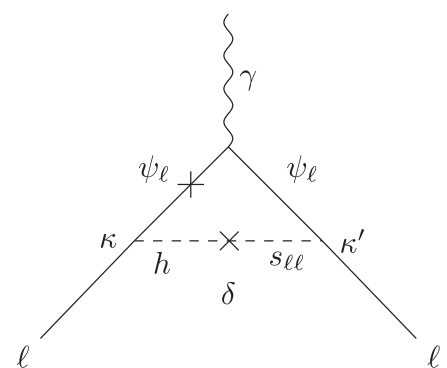

FIG. 21. Chirally enhanced contribution to the anomalous magnetic moment of a lepton $\ell$ through scalar mixing (cross on dashed line) and a $\psi_{\ell}$ chiral flip (cross on solid line).

the models $\mathrm{B}, \mathrm{D}, \mathrm{E}$, and $\mathrm{F}$ cannot accommodate either of the present data (68) and (69). Models A and C, on the other hand, have an additional diagram from the $S$ exchange, Fig. 19(b). In fact, since the $S$ field is a matrix in flavor space the unobserved flavor index of the BSM fermion $\psi_{i}$ in the loop makes this in total $N_{F}=3$ contributions. The external chirality flip again induces a contribution quadratic in lepton mass (70) which can account for $(g-2)_{\mu}$, since the coupling to the scalar singlet $\kappa^{\prime}$ is much less constrained than the one to the Higgs [40].

Certain NP scenarios, notably supersymmetric ones, can evade one power of lepton mass suppression in (70) by having instead the requisite chiral flip on the heavy fermion line in the loop, as in Fig. 21, such that

$$
a_{\ell}^{\mathrm{NP} *} \sim \alpha_{\eta} \frac{m_{\ell}}{M_{F}},
$$

opening up the possibility for larger contributions to $g-2$, and dipole operators in general. For $g-2$ we explore this further for models A and C in Sec. V F. Another application is electric dipole moments, discussed in Sec. V G.

\section{F. Scalar mixing and chiral enhancement}

The scalar potential involving the SM and BSM scalars $H$ and $S$ and the various quartic couplings and scalar mass terms has been given in (21). To investigate the prospect of chiral enhancement for dipole operators, such as those responsible for $(g-2)$, EDMs, or $\mu \rightarrow e \gamma$, we need to investigate the ground states. Using the methods of [12,51] two ground states $V^{ \pm}$have been identified in (22), including the conditions for couplings. The ground state $V^{+}$ respects flavor universality in interactions with the SM because it breaks $S U(3)_{\psi_{L}} \times S U(3)_{\psi_{R}} \rightarrow S U(3)_{\text {diag }}$ due to the diagonal $\operatorname{VEV}\left\langle S_{i j}\right\rangle=\frac{v_{s}}{\sqrt{2}} \delta_{i j}$. Conversely, $V^{-}$spontaneously violates flavor universality because it breaks $S U(3)_{\psi_{L}} \times S U(3)_{\psi_{R}}$ to $S U(2)_{\psi_{L}} \times S U(2)_{\psi_{R}} \times U(1)$ by only allowing a single diagonal component $k$ to pick up a nonvanishing $\operatorname{VEV}\left\langle S_{i j}\right\rangle=\frac{v_{s}}{\sqrt{2}} \delta_{i k} \delta_{j k}$.

If both scalars $S$ and $H$ acquire a VEV, the portal coupling $\delta$ induces a nondiagonal mass term in the potential which allows the scalars to mix. Together with both BSM Yukawas $\kappa, \kappa^{\prime}$ chiral enhancement can occur in models $\mathrm{A}$ and $\mathrm{C}$. A corresponding contribution to $g-2$ is shown in Fig. 21. First, we study the case $V^{-}$, where a single diagonal component of $S$ generates a VEV. The $S_{i i}$ component is chosen in order to target the generation $i$ of leptons in the term $\kappa^{\prime} \operatorname{Tr}\left[\bar{\psi}_{L} S E\right]$. We define

$H=\left(\begin{array}{c}h^{+} \\ \frac{1}{\sqrt{2}}\left(h+i h^{c}+v_{h}\right)\end{array}\right), \quad S_{i i}=\frac{1}{\sqrt{2}}\left(s_{i i}+i s_{i i}^{c}+v_{s}\right)$.

The mass matrix of the entire scalar sector is diagonal except for the mixing of $s_{i i}$ and $h$. Concentrating on this subsystem, the mass eigenstates $h_{1}, h_{2}$ can be expressed in terms of the mixing angle $\beta$ as

$$
\left(\begin{array}{l}
h_{1} \\
h_{2}
\end{array}\right)=\left(\begin{array}{cc}
\cos \beta & \sin \beta \\
-\sin \beta & \cos \beta
\end{array}\right)\left(\begin{array}{c}
s_{i i} \\
h
\end{array}\right) \text {, }
$$

where

$$
\tan 2 \beta=\frac{\delta}{\sqrt{\lambda(u+v)}} \frac{m_{h}}{m_{s}}\left(1+\mathcal{O}\left(m_{h}^{2} / m_{s}^{2}\right)\right)
$$

see Appendix D for details. Neglecting for the sake of this discussion the mixing induced by the scalar VEVs in the fermion system, the BSM Yukawa Lagrangian in the scalar mass basis reads

$$
\begin{aligned}
\mathcal{L}_{\beta}= & -\bar{\psi}_{j}\left[\left(\kappa \sin \beta \delta_{j k} P_{L}+\kappa^{\prime} \cos \beta \delta_{i j} \delta_{i k} P_{R}\right) h_{1}\right. \\
& \left.+\left(\kappa \cos \beta \delta_{j k} P_{L}-\kappa^{\prime} \sin \beta \delta_{i j} \delta_{i k} P_{R}\right) h_{2}\right] \ell_{k}+\text { H.c. },
\end{aligned}
$$

where we have again assumed $\kappa, \kappa^{\prime}$ real and $\kappa_{j k}=\kappa \delta_{j k}$. Provided that the mass eigenstate $h_{1}$ is much heavier than $h_{2}$ and $\psi$, and in the limit $M_{F} \gg m_{h_{2}}$, the leading contribution to $(g-2)_{\ell}$ reads, for $\ell=i$ with $\left\langle S_{i i}\right\rangle \neq 0$,

$$
a_{\ell}^{V^{-}}=-\frac{m_{\ell}}{2 M_{F}} \frac{\kappa \kappa^{\prime}}{16 \pi^{2}} \sin 2 \beta ;
$$

see Appendix B for details. This contribution is switched on only when both left and right chiral couplings of the lepton to the same scalar are present, a condition which is met by scalar mixing, and which comes with an enhancement factor $\frac{M_{F}}{m_{\ell}}\left(\frac{\alpha_{\kappa^{\prime}}}{\alpha_{\kappa}}\right)^{1 / 2}|\sin 2 \beta|$ with respect to NP contributions such as (70). $a_{\ell}^{V^{-}}$can have either sign.

If the vacuum is aligned in the muon direction, $(g-2)_{\mu}$ benefits from chiral enhancement (76). Figure 22 shows for which values of $M_{F},|\sin 2 \beta|$ the contribution to $(g-2)_{\mu}$ equals $\Delta a_{\mu}$ (68) for some benchmark values of $\sqrt{\alpha_{\kappa} \alpha_{\kappa^{\prime}}}$. Also shown is an upper limit on the mixing angle $\sin 2 \beta<$ 0.2 from Higgs signal strength measurements [62]. 


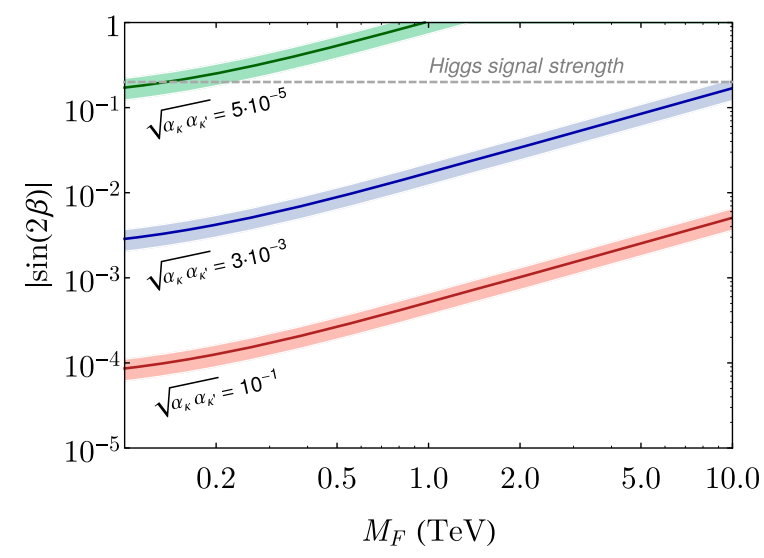

FIG. 22. The mixing angle $|\sin 2 \beta|$ as a function of $M_{F}$ that explains the $(g-2)_{\mu}$ anomaly within its $1 \sigma$ uncertainty (68) for a muon-aligned vacuum $V^{-}$(76) and for different values of $\sqrt{\alpha_{\kappa} \alpha_{\kappa^{\prime}}}$, with the upper bound from Higgs signal strength measurements [76]. For $V^{+}$the corresponding, requisite value of $\left|\sin 2 \beta^{\prime}\right|$ is a factor of $\sqrt{3}$ larger (78).

Next we consider the case $V^{+}$, where the BSM VEV is universal in all flavors. Here mixing occurs between the $h$ and the three $s_{i i}$ states acquiring the VEV. However, two of the mass eigenstates contain no component in the $h$ direction, and thus only mix the $s_{i i}$ states within themselves. The two normalized mass eigenstates that have nonvanishing components in the $h$ direction are

$h_{1}{ }^{\prime}=\frac{1}{\sqrt{3}}\left(\cos \beta^{\prime} s_{11}, \cos \beta^{\prime} s_{22}, \cos \beta^{\prime} s_{33}, \sqrt{3} \sin \beta^{\prime} h\right)$,

$h_{2}^{\prime}=\frac{-1}{\sqrt{3}}\left(\sin \beta^{\prime} s_{11}, \sin \beta^{\prime} s_{22}, \sin \beta^{\prime} s_{33},-\sqrt{3} \cos \beta^{\prime} h\right)$.

Hence, the mixing pattern with $h$ is identical for all $s_{i i}$. The enhanced contribution to the anomalous magnetic moments affects all lepton generations and reads

$$
a_{\ell}^{V^{+}}=-\frac{m_{\ell}}{2 \sqrt{3} M_{F}} \frac{\kappa \kappa^{\prime}}{16 \pi^{2}} \sin 2 \beta^{\prime}
$$

As $a_{\ell}^{V^{-}}$, it can a priori have either sign and can accommodate future $(g-2)_{\mu}$ data by adjusting $\kappa \kappa^{\prime} \delta / M_{F}$ together with the quartics. The parameter space $\beta^{\prime}, M_{F}$ that fits $\Delta a_{\mu}$ is, up to a factor of $\sqrt{3}$, the same as for $V^{-}$, shown in Fig. 22, and we note that this factor cancels with the one for the angle $\beta^{\prime}$, which obeys

$$
\tan 2 \beta^{\prime}=\frac{\sqrt{3} \delta}{\sqrt{\lambda u}} \frac{m_{h}^{\prime}}{m_{s}^{\prime}}\left(1+\mathcal{O}\left(m_{h}^{\prime 2} / m_{s}^{\prime 2}\right)\right)
$$

for $v=0$ and $\mu_{\text {det }}=0$; see Appendix D for details.

Owing to (78), we emphasize that fixing the parameter $\sin 2 \beta^{\prime}$ in $V^{+}$to explain $\Delta a_{\mu}$ in (68) also induces a contribution to the anomalous magnetic moments of the electron and the tau,

$$
\begin{aligned}
& a_{e}^{V^{+}}=\left(m_{e} / m_{\mu}\right) a_{\mu}^{V^{+}} \simeq 1.4 \times 10^{-11}, \\
& a_{\tau}^{V^{+}}=\left(m_{\tau} / m_{\mu}\right) a_{\mu}^{V^{+}} \simeq 4.5 \times 10^{-8} .
\end{aligned}
$$

The former, however, is in conflict with the data for $\Delta a_{e}$ in (69), both in magnitude and in sign, while the latter is 4 orders of magnitude away from present limits on $\Delta a_{\tau} \equiv$ $a_{\tau}^{\exp }-a_{\tau}^{\mathrm{SM}}[53]$.

On the other hand, larger couplings $\kappa^{\prime} / M_{F}[\mathrm{TeV}] \sim$ $\mathcal{O}(10)$ allow for a simultaneous explanation of both data points (68) and (69). This mechanism uses the diagrams in Fig. 19(b) to generate $\Delta a_{\mu}$, and the chirally enhanced diagram of Fig. 21 to generate $\Delta a_{e}$, without introducing flavor structure explicitly, and irrespective of the vacuum being flavor blind $\left(V^{+}\right)$or electron-aligned $\left(V^{-}\right.$with $\ell=e$ ). Moreover, the underlying mechanism is not finetuned and could, in principle, accommodate a wide range of deviations $\Delta a_{\mu}$ and $\Delta a_{e}$ different from present data.

Since the underlying Lagrangian does not break lepton flavor, this mechanism leads additionally to a prediction for the deviations of the tau anomalous magnetic moment $\Delta a_{\tau}$. Using the data (68) and (69), our models predict

$$
\Delta a_{\tau}^{V^{+}} \simeq(7.5 \pm 2.1) \times 10^{-7}
$$

if the vacuum is flavor blind, or

$$
\Delta a_{\tau}^{V^{-}} \simeq(8.1 \pm 2.2) \times 10^{-7}
$$

if the ground state is electron-aligned, respectively. Further details of this scenario can be found in [40].

Within our set of models, we conclude that the muon anomaly (68) alone, or the electron anomaly (69) alone, or both anomalies together, can only be explained by models $\mathrm{A}$ and $\mathrm{C}$.

\section{G. EDMs}

Unlike in the remainder of this work, here we allow the BSM Yukawas to be complex-valued. If the portal interaction $\delta$ is present, in models $\mathrm{A}$ and $\mathrm{C}$ a relative phase between $\kappa$ and $\kappa^{\prime}$ induces an electric dipole moment (EDM) of the SM leptons through the chirally enhanced one-loop diagram Fig. 21. The EDM-Lagrangian can be written as

$$
\mathcal{L}_{\mathrm{EDM}}=d_{\ell}(-i / 2) \bar{\ell} \sigma_{\mu \nu} \gamma_{5} F^{\mu \nu} \ell
$$

where $F^{\mu \nu}$ denotes the electromagnetic field strength tensor and $d_{\ell}$ the lepton electric dipole moment with mass dimension -1 . 
For model $\mathrm{A}$, and in the large- $M_{F}$ limit, we find

$$
\frac{d_{\ell}^{V^{-}}}{e}=-\frac{\sin 2 \beta}{4 M_{F}} \frac{\operatorname{Im}\left[\kappa^{*} \kappa^{\prime}\right]}{16 \pi^{2}}
$$

where the flavor-specific vacuum $V^{-}$is assumed with $\ell$ denoting the flavor distinguished by the ground state $\left(\left\langle S_{\ell \ell}\right\rangle \neq 0\right)$. Here, an EDM arises solely for the lepton flavor selected spontaneously by the vacuum. In turn, assuming the vacuum $V^{+}$and provided that the $C P$-phases are lepton universal, we find instead

$$
\frac{d_{\ell}^{V^{+}}}{e}=-\frac{\sin 2 \beta^{\prime}}{4 \sqrt{3} M_{F}} \frac{\operatorname{Im}\left[\kappa^{*} \kappa^{\prime}\right]}{16 \pi^{2}},
$$

for any flavor, and all EDMs are predicted to be equal. The same expressions (85), (86) and results hold true for model $\mathrm{C}$ except for the replacement $\kappa^{*} \kappa^{\prime} \rightarrow \kappa \kappa^{\prime *}$.

The current experimental bounds on $d_{e}$ and $d_{\mu}$,

$$
\begin{aligned}
& \left|d_{e}\right|<1.1 \times 10^{-29} e \mathrm{~cm}, \\
& \left|d_{\mu}\right|<1.5 \times 10^{-19} e \mathrm{~cm},
\end{aligned}
$$

by the ACME and Muon g-2 collaborations at $90 \%$ and 95\% C.L. [103,104], respectively, imply the bound

$$
\left|\sin 2 \beta \operatorname{Im}\left[\kappa^{*} \kappa^{\prime}\right]\right| / 16 \pi^{2}<2.2 \times 10^{-12}\left(M_{F} / \mathrm{TeV}\right),
$$

from the electron data, while the bound from muons is 10 orders of magnitude weaker. Comparing this to $\Delta a_{\mu}$ (68) induced by the same mechanism (see Fig. 22), the $C P$-phases must be suppressed at the order $10^{-7}\left(d_{e}\right.$ bound) and are unsuppressed by the muon EDM data. If the lepton EDMs are induced by a lepton flavor nonuniversal mechanism, by flavor-dependent $C P$-phases, or in a vacuum $V^{-}$pointing in the muon direction, the electron EDM bound could be bypassed and the muon EDM could be as large as $d_{\mu} \sim 2.5 \times 10^{-22} e \mathrm{~cm}$ given (68) with order one phases. Interestingly, this is in reach of future experiments $\left|d_{\mu}\right| \sim 5 \times 10^{-23} e \mathrm{~cm}[88]$.

\section{H. Charged LFV processes}

In the setup with Yukawa interactions (3), (7), and (8) flavor is conserved. While there is intergenerational mixing in Yukawas with $S$, no charged LFV proper occurs; see footnote 2. Here we envision a situation beyond (8) and allow for additional flavor off-diagonal couplings. Our aim is to see whether and how well such variants can be probed in LFV processes.

The $\ell_{i} \rightarrow \ell_{j} \gamma$ decay rate induced by a Higgs-fermion loop in all models except the singlet model $\mathrm{E}$ for $m_{\ell}, m_{h} \ll$ $M_{F}$ and $m_{j} \ll m_{i}$ can be written as [105]

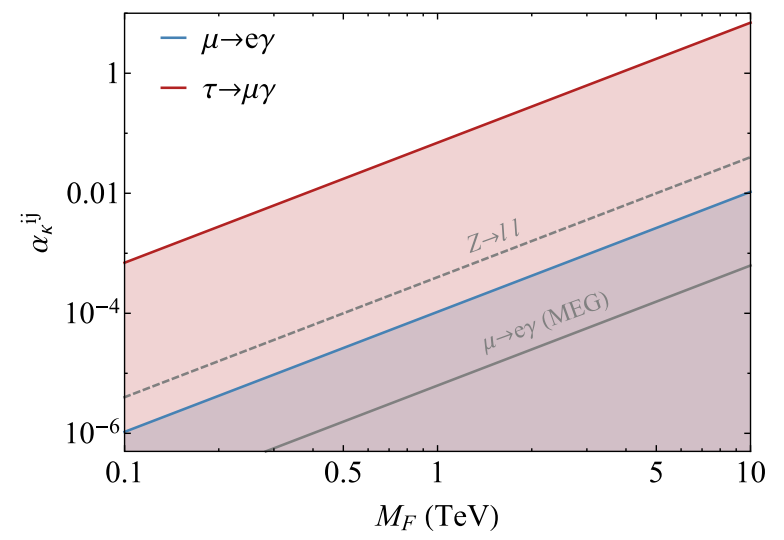

FIG. 23. Allowed regions (shaded) for $\alpha_{\kappa}^{\mu e}, \alpha_{\kappa}^{\tau \mu}$, and $M_{F}$ from LFV decays (91). Because of the proximity of upper limits on $\mathcal{B}(\tau \rightarrow e \gamma)$ and $\mathcal{B}(\tau \rightarrow \mu \gamma)$ only the latter is shown. The projected sensitivity of the MEG-II experiment [106] is shown by the solid gray line. The dashed gray line denotes the $Z \rightarrow \ell \ell$-constraint (64)

$$
\Gamma\left(\ell_{i} \rightarrow \ell_{j} \gamma\right)=\frac{\alpha_{e}}{576}\left(\alpha_{\kappa}^{i j}\right)^{2} \frac{m_{i}^{5}}{M_{F}^{4}}
$$

with

$$
\alpha_{\kappa}^{i j}=\frac{1}{(4 \pi)^{2}} \sum_{m} \kappa_{m i} \kappa_{m j}
$$

where $m$ corresponds to the flavors of the BSM fermion in the loop; see Appendix C for details. In (90), a flavor pattern proportional to $\kappa \delta_{i j}$ plus small off-diagonal entries of the order $\kappa \epsilon$ is assumed that is responsible for charged LFV. Hence, $\alpha_{\kappa}^{i j} \propto \alpha_{\kappa} \epsilon$, and $\Gamma\left(\ell_{i} \rightarrow \ell_{j} \gamma\right)$ arises at order $\epsilon^{2}$. Figure 23 shows how present bounds [53]

$$
\begin{aligned}
& \mathcal{B}(\mu \rightarrow e \gamma)<4.2 \times 10^{-13}, \\
& \mathcal{B}(\tau \rightarrow e \gamma)<3.3 \times 10^{-8}, \\
& \mathcal{B}(\tau \rightarrow \mu \gamma)<4.4 \times 10^{-8},
\end{aligned}
$$

at $90 \%$ C.L. and projected bounds $\mathcal{B}(\mu \rightarrow e \gamma) \lesssim 2 \times 10^{-15}$ from the MEG-II experiment [106] constrain $\alpha_{k}^{\mu e}, \alpha_{\kappa}^{\tau \mu}$ depending on $M_{F}$. Also shown for comparison is the constraint on diagonal couplings from $Z$ data (64). While present constraints on the off-diagonal entry $\alpha_{\kappa}^{\mu e}$ (blue line) are comparable to the diagonal ones from $Z \rightarrow \ell \ell$, the ones on $\alpha_{\kappa}^{\tau \mu}$ and $\alpha_{\kappa}^{\tau e}$ (red line) are significantly weaker.

LFV decays into three lepton final states are also possible, receiving contributions from both penguin and box diagrams with $\kappa, \kappa^{\prime}$. We estimate [107]

$$
\mathcal{B}(\mu \rightarrow e e \bar{e}) \sim \frac{3(4 \pi)^{2} \alpha_{e}^{2}}{8 G_{F}^{2}} \frac{\left(\alpha_{\kappa}^{\mu e}\right)^{2}}{M_{F}^{4}} \mathcal{B}\left(\mu \rightarrow e \bar{\nu}_{e} \nu_{\mu}\right),
$$


which is $\epsilon^{2}$ suppressed as (89). Present bounds from the SINDRUM collaboration $\mathcal{B}(\mu \rightarrow e e \bar{e})<10^{-12}$ [108] give $\alpha_{\kappa}^{\mu e} /\left(M_{F}[\mathrm{TeV}]\right)^{2}<(2-3) \times 10^{-4}$. This is indeed comparable with $\mu \rightarrow e \gamma$ bounds in Fig. 23, yet not more excluding. The parameter space will be further probed by the Mu3e experiment, which aims at a reach of $\mathcal{B}(\mu \rightarrow e e \bar{e})<10^{-16}$ [109]. For $\tau$ decays to three charged leptons, present bounds pose loose constraints on offdiagonal couplings, $\alpha_{\kappa}^{\tau \ell} /\left(M_{F}[\mathrm{TeV}]\right)^{2} \lesssim 0.1$.

On the other hand, $\mu$ to $e$ conversion processes have a limit in gold nuclei on the conversion rate (CR) of $\mathrm{CR}(\mu-e, \mathrm{Au}) \lesssim 7 \times 10^{-13}$ at $90 \%$ C.L. by the SINDRUM II collaboration [110]. In our models the conversion process is possible through $Z$ and $\gamma$ penguin contributions which receive $\epsilon^{2}$ suppression. We estimate $\mathrm{CR}(\mu-e, \mathrm{Au}) \sim \mathcal{O}\left(10^{-12}\right)\left(\alpha_{\kappa}^{\mu e} / 10^{-4}\right)^{2} /\left(M_{F}[\mathrm{TeV}]\right)^{4}$ [111], in close competition with $\mu \rightarrow e \gamma$ bounds. The future Mu2e experiment [112], with expected sensitivity $\mathrm{CR}(\mu-e, \mathrm{Au})<6.7 \times 10^{-17}$, can improve the bound from SINDRUM II on $\alpha_{\kappa}^{\mu e}$ by about 2 orders of magnitude.

Along the lines of the anomalous magnetic moments, scalar mixing induces chirally enhanced contributions to LFV dipole operators if $\kappa$ contains nonvanishing off-diagonal elements. For instance, the rate for $\mu \rightarrow e \gamma$ becomes

$$
\Gamma(\mu \rightarrow e \gamma)=\frac{\alpha_{e}}{64}\left(\frac{\kappa_{e \mu} \kappa^{\prime} \sin 2 \beta}{16 \pi^{2}}\right)^{2} \frac{m_{\mu}^{3}}{M_{F}^{2}},
$$

in the same approximations as in (76) and $V^{-}$pointing in the muon direction. (There is a similar contribution induced by $\kappa_{\mu e}$ which requires a scalar VEV in the electron direction.) Constraints on $\alpha_{\kappa \kappa^{\prime} \delta}^{\mu e}=\kappa_{e \mu} \kappa^{\prime} \sin 2 \beta /\left(16 \pi^{2}\right)$ from the chirally enhanced amplitude are stronger than on $\alpha_{\kappa}^{\mu e}$ by a factor $m_{\mu} / 3 M_{F}$.

\section{CONCLUSIONS}

We have studied SM extensions with three generations of vectorlike leptons and a new singlet matrix scalar field, inspired by asymptotic safety. The main focus has been on new physics implications for settings where the running couplings remain finite and well-defined at least up to the Planck scale, and possibly beyond. A key novelty over earlier models are Higgs and flavor portals (Table I) which are explored in depth. Within this setup we show that the number of new fermion generations required for asymptotically safe or Planck safe extensions can be much lower than thought previously.

Using the renormalization group, we have provided a comprehensive study of six basic models. All of them are found to be well-behaved up to the Planck scale in certain parameter regimes, owing to Yukawa couplings linking SM and BSM fermions with the Higgs (Figs. 7-10). The TeV scale initial conditions for BSM couplings (Fig. 13) illustrate parameter regions which do not run into Landau poles and vacuum instabilities or metastabilities. Similar results are found for models which admit a secondary Yukawa coupling between SM and BSM fermions and the new scalars (Figs. 11, 12) with a corresponding critical surface of parameters (Fig. 14). Very explicitly we learn that the requirement for safety up to the Planck scale provides a testable selection criterion in the BSM parameter space.

A conceptual novelty is the use of both top-down and bottom-up searches to find fixed points and Planck safe parameter regions. On the technical side, we have retained the RGEs for the gauge, Yukawa, and quartic couplings up to the complete two-loop order, extending upon previous studies. New features are walking regimes, and new patterns for fixed points are due to a competition between Yukawa, portal, and gauge couplings. Theories where the running of couplings can be extended to infinite energy are of interest in their own right. Our asymptotically safe extensions are the first ones that achieve this for the key SM and BSM couplings, and in accord with the measured values of the gauge couplings and the Higgs, top, and bottom masses.

Our models also offer a rich phenomenology due to their close ties with the SM through Yukawa and Higgs portals. Genuine features are LFV-like signatures in scalar decays

$$
S_{i j} \rightarrow \ell_{i}^{ \pm} \ell_{j}^{\mp}
$$

also with displaced vertices for sufficiently small coupling. The vectorlike leptons can have exotic charges that can lead to displaced vertex signatures. The models can be experimentally probed at colliders (Fig. 15), specifically through $\bar{\psi} \psi$ and Drell-Yan production, and additionally, at $e^{+} e^{-}$or $\mu^{+} \mu^{-}$machines, through single $\psi$ production $[113,114]$. The BSM scalars can be pair produced at lepton colliders, or in $p p$, if portal effects are present. It would be interesting to check whether existing new physics searches at the LHC in lepton-rich final states lead to constraints on model parameters. As no dedicated analysis for the models here has been performed, however, this requires a reinterpretation of existing searches that is beyond the scope of this work.

Finally, we comment on outstanding features related to lepton universality and low-energy probes for new physics, i.e., measurements of the lepton's magnetic or electric dipole moments. Except for the breaking by SM Yukawas, lepton universality is manifest in all our models and may or may not be broken spontaneously by the vacuum. Irrespective of the ground state, however, we find that two of the six basic models can explain the electron anomaly alone, the muon anomaly alone, or both anomalies together. The latter is rather remarkable in that it also entails a prediction for the tau anomalous magnetic moment [40], whereas any other BSM explanation of the muon and electron anomalies requires a manifest breaking of lepton universality [78-101]. In addition, provided the vacuum is 
flavorful and points into the muon direction, we find that the electron EDM bound can be bypassed with a sizable muon EDM at the level of $\sim 10^{-22} e \mathrm{~cm}$.

We look forward to further exploration of asymptotically safe model building and searches.

\section{ACKNOWLEDGMENTS}

G. H. and D. L. thank the Aspen Center for Physics, which is supported by National Science Foundation Grant No. PHY-1607611, for hospitality while part of this work was performed. Some results have been presented at the Moriond Electroweak conference 2019 and at the workshop "Asymptotic Safety meets Particle Physics" (Dortmund 2019). This work has been supported by the DFG Research Unit FOR 1873 "Quark Flavor Physics and Effective Field Theories."

\section{APPENDIX A: TWO-LOOP $\beta$-FUNCTIONS}

The following Appendixes collect technical details and useful formulas used within the main manuscript.

In this Appendix, we detail $\beta$-functions for the models A-F up to two-loop order. The results are based on [42-48].

The two-loop gauge and one-loop Yukawa RGEs can be expressed as

$$
\begin{aligned}
& \beta_{i}=-\alpha_{i}^{2}\left(B_{i}-\sum_{j=\text { gauge }} C_{i j} \alpha_{j}+\sum_{n=\text { Yukawa }} D_{\text {in }} \alpha_{n}\right), \\
& \beta_{n}=\alpha_{n}\left(\sum_{m=\text { Yukawa }} E_{n m} \alpha_{m}-\sum_{i=\text { gauge }} F_{n i} \alpha_{i}\right),
\end{aligned}
$$

corresponding to (13) and (14) in the main text. Some of the loop coefficients are model specific and are listed in Table VII, while others are universal or can be expressed in a general way in terms of the representation $R_{2}$ of the vectorlike fermions under $S U(2)_{L}$ and their hypercharge $Y$. In what follows, $C_{2}\left(R_{2}\right)$ denotes the quadratic Casimir invariant and $S_{2}\left(R_{2}\right)$ the Dynkin index (see [11] for details).

For the hypercharge coupling, these generic coefficients read

$$
\begin{aligned}
B_{1} & =-\frac{41}{3}-8 d\left(R_{2}\right) Y^{2}, \\
C_{11} & =\frac{199}{9}+24 d\left(R_{2}\right) Y^{4}, \\
C_{12} & =9+24 C_{2}\left(R_{2}\right) d\left(R_{2}\right) Y^{2}, \\
C_{13} & =\frac{88}{3}, \quad D_{1 t}=\frac{17}{3}, \quad D_{1 b}=\frac{5}{3}, \\
D_{1 y} & =36 d\left(R_{2}\right) Y^{2} .
\end{aligned}
$$

For the weak coupling, one obtains

$$
\begin{aligned}
B_{2} & =\frac{19}{3}-8 S_{2}\left(R_{2}\right), \\
C_{21} & =3+24 S_{2}\left(R_{2}\right) Y^{2}, \\
C_{22} & =\frac{35}{3}+12 S_{2}\left(R_{2}\right)\left(2 C_{2}\left(R_{2}\right)+20 / 3\right), \\
C_{23} & =24, \\
D_{2 t} & =D_{2 b}=3, \\
D_{2 y} & =36 S_{2}\left(R_{2}\right) .
\end{aligned}
$$

Finally, for the strong coupling the coefficients are inde-

\begin{tabular}{|c|c|c|c|c|c|c|c|c|c|c|c|c|c|c|c|c|}
\hline Model & $D_{1 \kappa}$ & $D_{1 \kappa^{\prime}}$ & $D_{2 \kappa}$ & $D_{2 \kappa^{\prime}}$ & $E_{t \kappa}$ & $E_{b \kappa}$ & $E_{y \kappa}$ & $E_{y K^{\prime}}$ & $E_{\kappa \kappa}$ & $F_{\kappa 1}$ & $F_{\kappa 2}$ & $E_{\kappa^{\prime} K^{\prime}}$ & $E_{\kappa^{\prime} y}$ & $E_{\kappa^{\prime} \kappa}$ & $F_{\kappa^{\prime} 1}$ & $F_{K^{\prime} 2}$ \\
\hline A & 15 & 36 & 3 & 0 & 6 & 6 & 2 & 8 & 9 & $15 / 2$ & $9 / 2$ & 8 & 8 & 0 & 12 & 0 \\
\hline B & $45 / 4$ & 0 & $33 / 4$ & 0 & $9 / 2$ & $9 / 2$ & $1 / 2$ & 0 & $23 / 4$ & $15 / 2$ & $33 / 2$ & & & & & \\
\hline C & 15 & 18 & 3 & 18 & 6 & 6 & 1 & 10 & 9 & $15 / 2$ & $9 / 2$ & 10 & 10 & 0 & 3 & 9 \\
\hline D & 39 & 0 & 3 & 0 & 6 & 6 & 1 & 0 & 9 & $39 / 2$ & $9 / 2$ & & & & & \\
\hline E & 3 & 0 & 3 & 0 & 6 & 6 & 2 & 0 & 9 & $3 / 2$ & $9 / 2$ & & & & & \\
\hline F & $9 / 4$ & 0 & $33 / 4$ & 0 & $9 / 2$ & $9 / 2$ & $1 / 2$ & 0 & $23 / 4$ & $3 / 2$ & $33 / 2$ & & & & & \\
\hline
\end{tabular}
pendent of the BSM sector

$$
\begin{aligned}
B_{3} & =14, & D_{3 t} & =4, \\
C_{31} & =\frac{11}{3}, & D_{3 b} & =4, \\
C_{32} & =9, & C_{33} & =-52 .
\end{aligned}
$$

For the Yukawa coefficients, we note that only $\alpha_{\kappa}$ couples into the one-loop running of the top and bottom Yukawas $\beta_{t, b}$; see Tables VII. Further loop coefficients for Yukawa couplings at one-loop are given by

$$
\begin{aligned}
E_{t t} & =E_{b b}=9, \quad E_{t b}=E_{b t}=3, \\
F_{t 1} & =\frac{17}{6}, \quad F_{b 1}=\frac{5}{6}, \\
F_{t 2} & =F_{b 2}=\frac{9}{2}, \quad F_{t 3}=F_{b 3}=16 .
\end{aligned}
$$

In a similar vein, there are no one-loop contributions from $\alpha_{3, t, b}$ to $\beta_{y}$ and $\beta_{\kappa^{\prime}}$. For $\beta_{y}$ one finds

TABLE VII. Model-specific loop coefficients for the gauge and Yukawa beta functions (A1). 
TABLE VIII. Model-specific loop coefficients for the quartic and Yukawa beta functions (A9), (A10), and (A12).

\begin{tabular}{lcccccccccc}
\hline \hline Model & $I_{\kappa}$ & $J_{\kappa \kappa}^{\lambda}$ & $K_{11 \kappa}^{\lambda}$ & $K_{12 \kappa}^{\lambda}$ & $K_{22 \kappa}^{\lambda}$ & $H_{\kappa \kappa \kappa}^{\lambda}$ & $H_{\kappa \kappa y}^{\delta}$ & $L_{1 \kappa}^{\lambda}$ & $L_{2 \kappa}^{\lambda}$ & $L_{1 y}^{\delta}$ \\
\hline $\mathrm{A}$ & 12 & 6 & $75 / 4$ & $-33 / 2$ & $9 / 4$ & 10 & 14 & 12 & 0 \\
$\mathrm{~B}$ & 9 & $15 / 8$ & $225 / 16$ & $-51 / 8$ & $-21 / 16$ & $47 / 32$ & $39 / 8$ & $15 / 4$ & $15 / 2$ & 9 \\
$\mathrm{C}$ & 12 & 6 & $75 / 4$ & $-33 / 2$ & $9 / 4$ & 10 & 16 & 12 & 0 & 6 \\
$\mathrm{D}$ & 12 & 6 & $219 / 4$ & $39 / 2$ & $9 / 4$ & 10 & 16 & 36 & 0 & 30 \\
$\mathrm{E}$ & 12 & 6 & $3 / 4$ & $3 / 2$ & $9 / 4$ & 10 & 14 & 0 & 0 & 0 \\
$\mathrm{~F}$ & 9 & $15 / 8$ & $9 / 16$ & $57 / 8$ & $-21 / 16$ & $47 / 32$ & $39 / 8$ & 0 & $15 / 2$ & 0 \\
\hline \hline
\end{tabular}

$$
\begin{aligned}
& E_{y y}=2\left(3+d\left(R_{2}\right)\right), \\
& F_{y 1}=12 Y^{2}, \\
& F_{y 2}=12 C_{2}\left(R_{2}\right) .
\end{aligned}
$$

Loop coefficients for $\beta_{\kappa}$ which are universal in all models are given by

$$
\begin{aligned}
E_{\kappa t} & =E_{\kappa b}=6, \\
E_{\kappa y} & =3, \\
E_{\kappa \kappa^{\prime}} & =F_{\kappa 3}=0,
\end{aligned}
$$

while those which are model specific are summarized in Table VII.

For the scalar couplings at one-loop, it is convenient to use the definition

$$
\tilde{\alpha}_{y}=\left\{\begin{array}{ll}
\alpha_{\kappa^{\prime}}+\alpha_{y} & \text { models A, C } \\
\alpha_{y} & \text { models B, D, E, F }
\end{array} .\right.
$$

In this convention, the one-loop RGEs for the scalar quartic couplings read

$$
\begin{aligned}
& \beta_{\lambda}^{(1)}=\beta_{\lambda}^{\mathrm{SM}(1)}+9 \alpha_{\delta}^{2}+I_{\kappa} \alpha_{\kappa} \alpha_{\lambda}-J_{\kappa \kappa}^{\lambda} \alpha_{\kappa}^{2}, \\
& \beta_{\delta}^{(1)}=\alpha_{\delta}\left[4 \alpha_{\delta}+12 \alpha_{\lambda}+24 \alpha_{u}+40 \alpha_{v}+6 \alpha_{t}+6 \alpha_{b}+\frac{1}{2} I_{\kappa} \alpha_{\kappa}+2 d\left(R_{2}\right) \tilde{\alpha}_{y}-\frac{3}{2} \alpha_{1}-\frac{9}{2} \alpha_{2}\right]-\frac{1}{3} I_{\kappa} \alpha_{\kappa} \alpha_{y}, \\
& \beta_{u}^{(1)}=24 \alpha_{u}\left(\alpha_{u}+\alpha_{v}\right)+2 d\left(R_{2}\right) \tilde{\alpha}_{y}\left(2 \alpha_{u}-\tilde{\alpha}_{y}\right), \\
& \beta_{v}^{(1)}=52 \alpha_{v}^{2}+12 \alpha_{u}\left(\alpha_{u}+4 \alpha_{v}\right)+2 \alpha_{\delta}^{2}+4 d\left(R_{2}\right) \tilde{\alpha}_{y} \alpha_{v} .
\end{aligned}
$$

Here, $\beta_{\lambda}^{\mathrm{SM}(1)}$ denotes the one-loop $\beta$-function of the Higgs quartic in the SM. The one-loop coefficients $I_{\kappa}$ and $J_{\kappa \kappa}^{\lambda}$ are tabulated in Table VIII.

At two-loop order, running of the couplings $\alpha_{t, b, \lambda}$ is modified via

$$
\begin{aligned}
\frac{\beta_{t}^{(2)}}{\alpha_{t}}= & \frac{\beta_{t}^{\mathrm{SM}(2)}}{\alpha_{t}}+9 \alpha_{\delta}^{2}-\frac{9}{4} J_{\kappa \kappa}^{\lambda} \alpha_{\kappa}^{2}-\frac{27}{24} I_{\kappa} \alpha_{\kappa}\left(\alpha_{y}+\alpha_{t}-\frac{15}{27} \alpha_{b}\right)+\frac{5}{4}\left(D_{1 \kappa} \alpha_{1}+3 D_{2 \kappa} \alpha_{2}\right) \alpha_{\kappa}+6 S_{2}\left(R_{2}\right) \alpha_{2}^{2}+\frac{58}{9} Y^{2} d\left(R_{2}\right) \alpha_{1}^{2}, \\
\frac{\beta_{b}^{(2)}}{\alpha_{b}}= & \frac{\beta_{b}^{\mathrm{SM}(2)}}{\alpha_{b}}+9 \alpha_{\delta}^{2}-\frac{9}{4} J_{\kappa \kappa}^{\lambda} \alpha_{\kappa}^{2}-\frac{27}{24} I_{\kappa} \alpha_{\kappa}\left(\alpha_{y}+\alpha_{b}-\frac{15}{27} \alpha_{t}\right)+\frac{5}{4}\left(D_{1 \kappa} \alpha_{1}+3 D_{2 \kappa} \alpha_{2}\right) \alpha_{\kappa}+6 S_{2}\left(R_{2}\right) \alpha_{2}^{2}-\frac{2}{9} Y^{2} d\left(R_{2}\right) \alpha_{1}^{2}, \\
\beta_{\lambda}^{(2)}= & \beta_{\lambda}^{\mathrm{SM}(2)}-90 \alpha_{\delta}^{2} \alpha_{\lambda}-36 \alpha_{\delta}^{3}-18 d\left(R_{2}\right) \tilde{\alpha}_{y} \alpha_{\delta}^{2}-12 I_{\kappa} \alpha_{\kappa} \alpha_{\lambda}^{2}-\frac{1}{2} J_{\kappa \kappa}^{\lambda} \alpha_{\kappa}^{2} \alpha_{\lambda}-\frac{27}{12} I_{\kappa} \alpha_{\kappa} \alpha_{y} \alpha_{\lambda}+3 J_{\kappa \kappa}^{\lambda} \alpha_{y} \alpha_{\kappa}^{2}+3 H_{\kappa \kappa \kappa}^{\lambda} \alpha_{\kappa}^{3} \\
& -L_{1 \kappa}^{\lambda} \alpha_{1} \alpha_{\kappa}^{2}-L_{2 \kappa}^{\lambda} \alpha_{2} \alpha_{\kappa}^{2}+\frac{5}{2}\left(D_{1 \kappa} \alpha_{1}+3 D_{2 \kappa} \alpha_{2}\right) \alpha_{\kappa} \alpha_{\lambda}-K_{11 \kappa}^{\lambda} \alpha_{1}^{2} \alpha_{\kappa}-K_{12 \kappa}^{\lambda} \alpha_{1} \alpha_{2} \alpha_{\kappa}-K_{22 \kappa}^{\lambda} \alpha_{2}^{2} \alpha_{\kappa} \\
& +30 S_{2}\left(R_{2}\right) \alpha_{2}^{2} \alpha_{\lambda}+10 d\left(R_{2}\right) Y^{2} \alpha_{1}^{2} \alpha_{\lambda}-4 d\left(R_{2}\right) Y^{2}\left(\alpha_{1}+\alpha_{2}\right) \alpha_{1}^{2}-4 S_{2}\left(R_{2}\right)\left(\alpha_{1}+3 \alpha_{2}\right) \alpha_{2}^{2},
\end{aligned}
$$

using coefficients in Tables VII and VIII, and $\beta_{t, b, \lambda}^{\mathrm{SM}(2)}$ denote the two-loop beta functions of the SM. 
TABLE IX. Model-specific two-loop coefficients for the BSM Yukawa beta functions (A11).

\begin{tabular}{lccccccccc}
\hline \hline Model & $P_{\kappa \kappa}^{y}$ & $P_{\kappa \kappa}^{\kappa}$ & $Q_{1 \kappa}^{y}$ & $Q_{2 \kappa}^{y}$ & $Q_{1 y}^{\kappa}$ & $Q_{2 \kappa}^{\kappa}$ & $Q_{1 \kappa}^{\kappa}$ & $Q_{12}^{\kappa}$ & $R_{11}^{\kappa}$ \\
\hline $\mathrm{A}$ & $19 / 2$ & 24 & $37 / 4$ & $51 / 4$ & 6 & $225 / 8$ & $279 / 8$ & $9 / 2$ & $721 / 12$ \\
$\mathrm{~B}$ & $57 / 32$ & $59 / 8$ & $37 / 16$ & $-101 / 16$ & 6 & $1343 / 32$ & $785 / 32$ & $-3 / 2$ & $1249 / 12$ \\
$\mathrm{C}$ & 5 & 24 & $55 / 8$ & $33 / 8$ & 15 & $225 / 8$ & $279 / 8$ & $9 / 2$ & $589 / 12$ \\
$\mathrm{D}$ & 5 & 24 & $95 / 8$ & $33 / 8$ & 27 & $225 / 8$ & $831 / 8$ & $27 / 2$ & $4541 / 12$ \\
$\mathrm{E}$ & $19 / 2$ & 24 & $17 / 4$ & $51 / 4$ & 0 & $225 / 8$ & $123 / 8$ & $-9 / 2$ & $35 / 12$ \\
$\mathrm{~F}$ & $57 / 32$ & $59 / 8$ & $17 / 4$ & $-101 / 16$ & 0 & $1343 / 32$ & $205 / 32$ & $3 / 2$ & $35 / 12$ \\
\hline \hline
\end{tabular}

Two-loop RGEs of $\alpha_{y, k, \kappa^{\prime}}$ read

$$
\begin{aligned}
\frac{\beta_{\kappa}^{(2)}}{\alpha_{\kappa}}= & -P_{\kappa \kappa}^{\kappa} \alpha_{\kappa}^{2}-\frac{9}{4}\left(1+2 d\left(R_{2}\right)\right) \tilde{\alpha}_{y} \alpha_{y}-\frac{3}{32}\left[90+d\left(R_{2}\right)\left(89-27 d\left(R_{2}\right)\right)\right] \alpha_{y} \alpha_{\kappa}+R_{11}^{\kappa} \alpha_{1}^{2} \\
& -\frac{1}{6}\left[49+d\left(R_{2}\right)\left(39-19 d\left(R_{2}\right)\right)\right] \alpha_{2}^{2}+Q_{1 y}^{\kappa} \alpha_{1} \alpha_{y}+Q_{2 \kappa}^{\kappa} \alpha_{2} \alpha_{\kappa}+Q_{12}^{\kappa} \alpha_{1} \alpha_{2}+Q_{1 \kappa}^{\kappa} \alpha_{1} \alpha_{\kappa} \\
& +\frac{5}{12} \alpha_{1}\left[17 \alpha_{t}+5 \alpha_{b}\right]+\frac{45}{4} \alpha_{2}\left[\alpha_{t}+\alpha_{b}\right]+40 \alpha_{3}\left[\alpha_{t}+\alpha_{b}\right]-6 S_{2}\left(R_{2}\right)\left[1-2 d\left(R_{2}\right)\right] \alpha_{2} \alpha_{y} \\
& -\frac{27}{2}\left[\alpha_{t}^{2}+\alpha_{b}^{2}\right]+3 \alpha_{t} \alpha_{b}-J_{\kappa \kappa}^{\lambda} I_{\kappa}^{-1}\left(27 \alpha_{t}+27 \alpha_{b}+48 \alpha_{\lambda}\right) \alpha_{\kappa} \\
& -12 \alpha_{y} \alpha_{\delta}+9 \alpha_{\delta}^{2}+12 \alpha_{\lambda}^{2}, \\
\frac{\beta_{\kappa^{\prime}}^{(2)}=}{\alpha_{\kappa^{\prime}}}= & {\left[\frac{211}{3}+2 Y^{2}\left(20 d\left(R_{2}\right)-3\right)\right] Y^{2} \alpha_{1}^{2}-\left[\frac{257}{3}+6 C_{2}\left(R_{2}\right)-40 S_{2}\left(R_{2}\right)\right] C_{2}\left(R_{2}\right) \alpha_{2}^{2} } \\
& -12 C_{2}\left(R_{2}\right) Y^{2} \alpha_{1} \alpha_{2}+\left[48+10 d\left(R_{2}\right)\right] Y^{2} \alpha_{1} \tilde{\alpha}_{y}+\left[48+10 d\left(R_{2}\right)\right] C_{2}\left(R_{2}\right) \alpha_{2} \tilde{\alpha}_{y} \\
& +8\left[5 \alpha_{u}^{2}+5 \alpha_{v}^{2}+6 \alpha_{u} \alpha_{v}\right]+2 \alpha_{\delta}^{2}-16\left(5 \alpha_{u}+3 \alpha_{v}\right) \tilde{\alpha}_{y}-\left[\frac{1}{2}+18 d\left(R_{2}\right)\right] \tilde{\alpha}_{y}^{2} \\
& -P_{\kappa \kappa}^{y} \alpha_{\kappa}^{2}+Q_{1 \kappa}^{y} \alpha_{1} \alpha_{\kappa}+Q_{2 \kappa}^{y} \alpha_{2} \alpha_{\kappa}-\frac{3}{2^{d\left(R_{2}\right)}}\left(2 d\left(R_{2}\right)+1\right) \alpha_{y} \alpha_{\kappa}, \\
\frac{\beta_{y}^{(2)}=}{\alpha_{y}}= & {\left[\frac{211}{3}+2 Y^{2}\left(20 d\left(R_{2}\right)-3\right)\right] Y^{2} \alpha_{1}^{2}-\left[\frac{257}{3}+6 C_{2}\left(R_{2}\right)-40 S_{2}\left(R_{2}\right)\right] C_{2}\left(R_{2}\right) \alpha_{2}^{2} } \\
& -12 C_{2}\left(R_{2}\right) Y^{2} \alpha_{1} \alpha_{2}+\left[48+10 d\left(R_{2}\right)\right] Y^{2} \alpha_{1} \tilde{\alpha}_{y}+\left[48+10 d\left(R_{2}\right)\right] C_{2}\left(R_{2}\right) \alpha_{2} \tilde{\alpha}_{y} \\
& +8\left[5 \alpha_{u}^{2}+5 \alpha_{v}^{2}+6 \alpha_{u} \alpha_{v}\right]+2 \alpha_{\delta}^{2}-16\left(5 \alpha_{u}+3 \alpha_{v}\right) \tilde{\alpha}_{y}-\left[\frac{1}{2}+18 d\left(R_{2}\right)\right] \tilde{\alpha}_{y}^{2} \\
& -P_{\kappa \kappa}^{y} \alpha_{\kappa}^{2}+Q_{1 \kappa}^{y} \alpha_{1} \alpha_{\kappa}+Q_{2 \kappa}^{y} \alpha_{2} \alpha_{\kappa} \\
& -2^{-d\left(R_{2}\right)}\left[18 \alpha_{t}+18 \alpha_{b}+3\left(2 d\left(R_{2}\right)+1\right) \alpha_{y}+16 \alpha_{\delta}\right] \alpha_{\kappa},
\end{aligned}
$$

also using the loop coefficients tabulated in Table IX.

Finally, the two-loop contributions for the BSM scalar quartics are 


$$
\begin{aligned}
& \beta_{u}^{(2)}=-336 \alpha_{u}^{3}-1056 \alpha_{u}^{2} \alpha_{v}-688 \alpha_{u} \alpha_{v}^{2}+\left[Y^{2} d\left(R_{2}\right) \alpha_{1}+3 S_{2}\left(R_{2}\right) \alpha_{2}\right]\left[20 \alpha_{u}-8 \tilde{\alpha}_{y}\right] \tilde{\alpha}_{y} \\
& -48 d\left(R_{2}\right) \tilde{\alpha}_{y}\left(\alpha_{u}+\alpha_{v}\right) \alpha_{u}+2 d\left(R_{2}\right)\left[6 \tilde{\alpha}_{y}-9 \alpha_{u}+4 \alpha_{v}\right] \tilde{\alpha}_{y}-20 \alpha_{\delta}^{2} \alpha_{u}+I_{\kappa} \alpha_{\kappa} \alpha_{y}\left[\frac{1}{3} \tilde{\alpha}_{y}-\frac{1}{2} \alpha_{u}\right] \text {, } \\
& \beta_{v}^{(2)}=-288 \alpha_{u}^{3}-688 \alpha_{u}^{2} \alpha_{v}-1056 \alpha_{u} \alpha_{v}^{2}-816 \alpha_{v}^{3}+20\left[Y^{2} d\left(R_{2}\right) \alpha_{1}+3 S_{2}\left(R_{2}\right) \alpha_{2}\right] \tilde{\alpha}_{y} \alpha_{v} \\
& -24 d\left(R_{2}\right)\left[\alpha_{u}^{2}+4 \alpha_{v} \alpha_{u}+\frac{13}{3} \alpha_{v}^{2}\right] \tilde{\alpha}_{y}+2 d\left(R_{2}\right)\left[4 \alpha_{u}-9 \alpha_{v}+2 \tilde{\alpha}_{y}\right] \tilde{\alpha}_{y}^{2}-\frac{1}{2} I_{\kappa} \alpha_{\kappa} \alpha_{y} \alpha_{v} \\
& +4\left[\alpha_{1}+3 \alpha_{2}-3 \alpha_{t}-3 \alpha_{b}-\frac{1}{4} I_{\kappa} \alpha_{\kappa}-5 \alpha_{v}-2 \alpha_{\delta}\right] \alpha_{\delta}^{2} \text {, } \\
& \beta_{\delta}^{(2)}=\left[\frac{557}{48}+5 Y^{2} d\left(R_{2}\right)\right] \alpha_{1}^{2} \alpha_{\delta}+\frac{15}{8} \alpha_{1} \alpha_{2} \alpha_{\delta}+\left[-\frac{145}{16}+15 S_{2}\left(R_{2}\right)\right] \alpha_{2}^{2} \alpha_{\delta}+\left[\frac{85}{12} \alpha_{t}+\frac{25}{12} \alpha_{b}\right] \alpha_{1} \alpha_{\delta} \\
& +\frac{45}{4}\left[\alpha_{t}+\alpha_{b}\right] \alpha_{2} \alpha_{\delta}+40\left[\alpha_{t}+\alpha_{b}\right] \alpha_{3} \alpha_{\delta}+10\left[Y^{2} d\left(R_{2}\right) \alpha_{1}+3 S_{2}\left(R_{2}\right) \alpha_{2}\right] \tilde{\alpha}_{y} \alpha_{\delta} \\
& -\frac{27}{2}\left[\alpha_{t}^{2}+\alpha_{b}^{2}\right] \alpha_{\delta}-21 \alpha_{t} \alpha_{b} \alpha_{\delta}-d\left(R_{2}\right)\left[48 \alpha_{u}+80 \alpha_{v}+9 \tilde{\alpha}_{y}\right] \tilde{\alpha}_{y} \alpha_{\delta}-\frac{17}{24} I_{\kappa} \alpha_{\kappa} \alpha_{y} \alpha_{\delta}-\frac{27}{12} J_{\kappa \kappa}^{\lambda} \alpha_{\kappa}^{2} \alpha_{\delta} \\
& -200\left[\alpha_{u}^{2}+\frac{6}{5} \alpha_{u} \alpha_{v}+\alpha_{v}^{2}\right] \alpha_{\delta}+12\left[2 \alpha_{1}+6 \alpha_{2}-6 \alpha_{t}-6 \alpha_{b}-\frac{1}{2} I_{\kappa} \alpha_{\kappa}-5 \alpha_{\lambda}\right] \alpha_{\lambda} \alpha_{\delta} \\
& +\left[\alpha_{1}+3 \alpha_{2}-12 \alpha_{t}-12 \alpha_{b}-144 \alpha_{u}-240 \alpha_{v}-I_{\kappa} \alpha_{\kappa}-4 d\left(R_{2}\right) \tilde{\alpha}_{y}-19 \alpha_{\delta}-72 \alpha_{\lambda}\right] \alpha_{\delta}^{2} \\
& +\frac{15}{4}\left[\frac{1}{3} D_{1 \kappa} \alpha_{1}+D_{2 \kappa} \alpha_{2}\right] \alpha_{\kappa} \alpha_{\delta}+\frac{5}{2} I_{\kappa} \alpha_{\kappa} \alpha_{y} \tilde{\alpha}_{y}+H_{\kappa \kappa y}^{\delta} \alpha_{\kappa}^{2} \alpha_{y} \\
& -12 Y^{2} d\left(R_{2}\right) \alpha_{1}^{2} \tilde{\alpha}_{y}-36\left[\frac{5}{3} d\left(R_{2}\right)-4\right] S_{2}\left(R_{2}\right) \alpha_{2}^{2} \tilde{\alpha}_{y}-L_{1 y}^{\delta} \alpha_{1} \alpha_{y} \alpha_{\kappa}-L_{2 y}^{\delta} \alpha_{2} \alpha_{y} \alpha_{\kappa} \text {, }
\end{aligned}
$$

with model-specific loop coefficients tabulated in Table VIII.

\section{APPENDIX B: BSM CONTRIBUTIONS TO $g-2$}

Results for weak corrections to $g-2$ in general gauge models can be found in [115]. In this work the relevant BSM contribution comes from a neutral scalar- $\psi$ loop. Using the general Yukawa Lagrangian with chiral projectors $P_{L / R}=\left(1 \mp \gamma_{5}\right) / 2$

$$
\mathcal{L}_{Y}=\bar{\psi}\left(c_{L i} P_{L}+c_{R i} P_{R}\right) \ell_{i} H+\text { H.c. },
$$

where $\psi$ is a fermion with charge $Q_{F}=-1, H$ is a neutral scalar, and $\ell_{i}$ is a charged lepton of flavor $i$,

$$
\begin{aligned}
a_{i}^{\mathrm{NP}}= & \frac{m_{i}^{2}}{8 \pi^{2}} \int_{0}^{1} \mathrm{~d} x \frac{\frac{1}{2}\left(c_{L i}^{2}+c_{R i}^{2}\right)\left(x^{2}-x^{3}\right)+\frac{M_{F}}{m_{i}} c_{L i} c_{R i} x^{2}}{m_{i}^{2} x^{2}+\left(M_{F}^{2}-m_{i}^{2}\right) x+m_{H}^{2}(1-x)} \\
= & \frac{m_{i}^{2}}{16 \pi^{2} m_{H}^{2}}\left[\frac{1}{2}\left(c_{L i}^{2}+c_{R i}^{2}\right) I_{1}\left(M_{F}^{2} / m_{H}^{2}\right)\right. \\
& \left.+\frac{M_{F}}{m_{i}} c_{L i} c_{R i} I_{2}\left(M_{F}^{2} / m_{H}^{2}\right)\right]
\end{aligned}
$$

where we assumed real couplings $c_{L i}, c_{R i}$. For $m_{i} \rightarrow 0$ in the integrals with $t=M_{F}^{2} / m_{H}^{2}$ one obtains

$$
\begin{aligned}
& I_{1}(t)=\frac{t^{3}-6 t^{2}+3 t+6 t \ln (t)+2}{3(t-1)^{4}}, \\
& I_{2}(t)=\frac{t^{2}-4 t+2 \ln (t)+3}{(t-1)^{3}} .
\end{aligned}
$$

The limits $t \rightarrow \infty$ (heavier fermion) and $t \rightarrow 0$ (heavier scalar) yield

$$
\begin{aligned}
& a_{i}^{\mathrm{NP}, F}=\frac{1}{16 \pi^{2}} \frac{m_{i}^{2}}{M_{F}^{2}}\left[\frac{1}{6}\left(c_{L i}^{2}+c_{R i}^{2}\right)+\frac{M_{F}}{m_{i}} c_{L i} c_{R i}\right], \\
& a_{i}^{\mathrm{NP}, H}=\frac{1}{16 \pi^{2}} \frac{m_{i}^{2}}{m_{H}^{2}}\left[\frac{1}{3}\left(c_{L i}^{2}+c_{R i}^{2}\right)-\frac{M_{F}}{m_{i}} c_{L i} c_{R i}\left(2 \ln \frac{M_{F}^{2}}{m_{H}^{2}}+3\right)\right],
\end{aligned}
$$

respectively. For $t=1$, the integrals are well-defined, $I_{1}(1)=1 / 6, I_{2}(1)=2 / 3$. The presence of both Yukawas $c_{L i}, c_{R i} \neq 0$ switches on the rightmost terms in (B4) with enhancement factors $M_{F} / m_{i}$.

\section{APPENDIX C: LFV BRANCHING RATIOS}

Here we provide the $\ell_{i} \rightarrow \ell_{j} \gamma$ decay rate mediated by Yukawa interactions with a neutral scalar for a general 
Lagrangian (B1). We consider only the cases where either the fermion $F$ or the boson $H$ propagating in the loop are much heavier than the leptons. If the interaction is purely left- or right-handed (either $c_{L i}=0$ or $c_{R i}=0$ for all $i$ ), the decay rate is [105]

$$
\begin{aligned}
& \Gamma\left(\ell_{i} \rightarrow \ell_{j} \gamma\right) \\
& \quad=\frac{\alpha_{e}}{4 m_{i}^{3}}\left(m_{i}^{2}-m_{j}^{2}\right)^{3}\left(m_{i}^{2}+m_{j}^{2}\right)\left(c_{X j}^{*} c_{X i}\right)^{2}\left|F_{1}\left(M_{F}^{2} / m_{H}^{2}\right)\right|^{2},
\end{aligned}
$$

where $X=L, R$, and $F(t)$ in the limit $m_{i}^{2}, m_{j}^{2} \rightarrow 0$ reads

$$
F_{1}(t)=\frac{i}{16 \pi^{2} m_{H}^{2}}\left[\frac{t^{2}-5 t-2}{12(t-1)^{3}}+\frac{t \ln (t)}{2(t-1)^{4}}\right] .
$$

Taking $t \rightarrow \infty$ and $m_{i} \gg m_{j}$ one recovers Eq. (89). For a scalar more massive than the fermion $F$, taking $t \rightarrow 0$ and $m_{i} \gg m_{j}$ we obtain

$$
\Gamma^{H}\left(\ell_{i} \rightarrow \ell_{j} \gamma\right)=\frac{\alpha_{e}}{144}\left(\frac{c_{X j}^{*} c_{X i}}{16 \pi^{2}}\right)^{2} \frac{m_{i}^{5}}{m_{H}^{4}}
$$

If both left- and right-handed interactions are present, the leading contribution reads

$$
\begin{aligned}
\Gamma\left(\ell_{i} \rightarrow \ell_{j} \gamma\right)= & \frac{\alpha_{e}}{4 m_{i}^{3}}\left(m_{i}^{2}-m_{j}^{2}\right)^{3} M_{F}^{2}\left|F_{2}\left(M_{F}^{2} / m_{H}^{2}\right)\right|^{2} \\
& \times\left[\left(c_{R j}^{*} c_{L i}\right)^{2}+\left(c_{L j}^{*} c_{R i}\right)^{2}\right],
\end{aligned}
$$

with

$$
F_{2}(t)=\frac{i}{16 \pi^{2} m_{H}^{2}}\left[\frac{t-3}{2(t-1)^{2}}+\frac{\ln (t)}{(t-1)^{3}}\right] .
$$

For $t \rightarrow 0, t \rightarrow \infty$ and $m_{i} \gg m_{j}$ yields, respectively,

$$
\begin{aligned}
\Gamma^{F}\left(\ell_{i} \rightarrow \ell_{j} \gamma\right)= & \frac{\alpha_{e}}{16}\left[\left(\frac{c_{R j}^{*} c_{L i}}{16 \pi^{2}}\right)^{2}+\left(\frac{c_{L j}^{*} c_{R i}}{16 \pi^{2}}\right)^{2}\right] \frac{m_{i}^{3}}{M_{F}^{2}}, \\
\Gamma^{H}\left(\ell_{i} \rightarrow \ell_{j} \gamma\right)= & \frac{\alpha_{e}}{4}\left[\left(\frac{c_{R j}^{*} c_{L i}}{16 \pi^{2}}\right)^{2}+\left(\frac{c_{L j}^{*} c_{R i}}{16 \pi^{2}}\right)^{2}\right] \\
& \times \frac{m_{i}^{3} M_{F}^{2}}{m_{H}^{4}}\left(\frac{3}{2}+\ln \frac{M_{F}^{2}}{m_{H}^{2}}\right)^{2} .
\end{aligned}
$$

Here we neglected terms proportional to $\left(c_{X j}^{*} c_{X i}\right)^{2}$; assuming $\left(c_{X j}^{*} c_{X i}\right)^{2}=\mathcal{O}\left(c_{R j}^{*} c_{L i}, c_{L j}^{*} c_{R i}\right)^{2}$, the results (C4)-(C6) are valid up to corrections of order $m_{i} / M_{F}$. The results apply for the BSM Yukawa couplings with the physical Higgs in models A, C, D and in model B for $\alpha_{\kappa}^{i j} \rightarrow \alpha_{\kappa}^{i j} / 2$.
APPENDIX D: MASS MATRICES AND SCALAR POTENTIAL

The VEVs in terms of the parameters of the potential (21) are obtained as

$$
\begin{aligned}
& v_{s}^{2}=\frac{\mu_{s}^{2}-\frac{\delta}{2 \lambda} \mu^{2}}{u+n v-n \frac{\delta^{2}}{4 \lambda}}, \\
& v_{h}^{2}=\frac{\mu^{2}-\frac{\delta n}{2(u+n v)} \mu_{s}^{2}}{\lambda-n \frac{\delta^{2}}{4(u+n v)}}=\frac{1}{\lambda}\left(\mu^{2}-n \delta \frac{v_{s}^{2}}{2}\right),
\end{aligned}
$$

with $n=1,3$ for the vacuum solutions $V^{-}$and $V^{+}$, respectively. If the trilinear term $\mu_{\text {det }}$ is switched on, for $V^{+}$one should replace $\mu_{s}^{2} \rightarrow \mu_{s}^{2}+\mu_{\mathrm{det}} v_{s} / \sqrt{2}$ and solve accordingly for $v_{s}$. A detailed analysis of the vacuum structure can be found in [116] for a similar case. Before the scalars acquire these VEVs the potential is symmetric under the transformation $S \rightarrow U_{\psi_{L}} S U_{\psi_{R}}^{\dagger}$, where $U_{i}$ are $3 \times 3$ unitary matrices, each with 9 degrees of freedom. In the case of a muon-aligned $V^{-}$, the VEV in $s_{22}$ breaks this symmetry into $U(2)_{\psi_{L}} \times U(2)_{\psi_{R}} \times U(1)$. The number of massless modes in $S$ is then $2 \times 9-2 \times 4-1=9$. In $V^{+}$, the universal VEVs break $U(3)_{\psi_{L}} \times U(3)_{\psi_{R}} \rightarrow U(3)_{\text {diag, }}$, yielding 9 Goldstone modes as well. We assume that additional mass terms prohibit the presence of massless Goldstones. The symmetries involving the Higgs are the same as in the SM, rendering three massless states, which are eaten by $W^{ \pm}, Z$.

The $S-H$ mixing in the mass Lagrangean $V^{\text {mass }}$ in vacuum $V^{-}$is obtained from

$$
\begin{aligned}
\left.\frac{\partial^{2} V}{\partial h \partial h}\right|_{S, H=0} & =m_{h}^{2}=-\mu^{2}+3 v_{h}^{2} \lambda+\frac{1}{2} \delta v_{s}^{2} \\
& =\frac{2(u+v) \mu^{2}-\delta \mu_{s}^{2}}{(u+v)-\delta^{2} / 4 \lambda}, \\
\left.\frac{\partial^{2} V}{\partial s_{22} \partial s_{22}}\right|_{S, H=0} & =m_{s}^{2}=-\mu_{s}^{2}+3 v_{s}^{2}(u+v)+\frac{1}{2} \delta v_{h}^{2} \\
& =\frac{2 \lambda \mu_{s}^{2}-\delta \mu^{2}}{\lambda-\delta^{2} / 4(u+v)}, \\
\left.\frac{\partial^{2} V}{\partial h \partial s_{22}}\right|_{S, H=0} & =m_{s h}=\delta v_{s} v_{h} \\
& =\frac{\delta}{2 \sqrt{\lambda(u+v)}} m_{s} m_{h} .
\end{aligned}
$$

Thus, $h$ and $s_{22}$ mix according to

$V^{\text {mass }}\left(s_{22}, h\right)=\frac{1}{2}\left(s_{22}, h\right)\left(\begin{array}{cc}m_{s}^{2} & m_{s h} \\ m_{s h} & m_{h}^{2}\end{array}\right)\left(\begin{array}{c}s_{22} \\ h\end{array}\right)$,

with eigenvalues 


$$
m_{2}=\frac{1}{2}\left[m_{s}^{2}+m_{h}^{2} \pm \sqrt{\left(m_{s}^{2}-m_{h}^{2}\right)^{2}+4 m_{s h}^{2}}\right]
$$

The masses of the fields which do not get a finite VEV are obtained as

$$
\begin{aligned}
\left.\frac{\partial^{2} V}{\partial\left(S_{i i}^{2}\right)}\right|_{S, H=0} & =\bar{m}_{s}^{2}=-\mu_{s}^{2}+v_{s}^{2} v+\frac{1}{2} \delta v_{h}^{2} \\
& =-\frac{u}{2(u+v)} m_{s}^{2} \quad \text { for } i=1,3 .
\end{aligned}
$$

Note $\bar{m}_{s}^{2}$ is positive since $u<0$ for $V^{-}(22)$.

The mass eigenstates $h_{1}, h_{2}$ can be expressed as in (73) in terms of the angle $\beta$, where

$$
\tan 2 \beta=\frac{2 m_{s h}}{m_{s}^{2}-m_{h}^{2}}=\frac{2 \delta v_{h} v_{s}}{m_{s}^{2}-m_{h}^{2}} .
$$

Expanding for $m_{h} \ll m_{s}$ yields (74).

The VEVs of the $S$ and $H$ scalars induce mixing between the BSM fermions and the leptons. Defining $f_{X}=\left(e_{X}, \mu_{X}, \tau_{X}, \psi_{X 1}, \psi_{X 2}, \psi_{X 3}\right)^{T}, X=L, R$, the corresponding mass mixing term for model A can be written as

$$
\begin{aligned}
\bar{f}_{L} \mathcal{M}_{f} f_{R}= & \frac{v_{h}}{\sqrt{2}} \bar{e}_{L} Y_{e} e_{R}+\frac{v_{h}}{\sqrt{2}} \kappa \bar{e}_{L} \psi_{R}+\frac{v_{s}}{\sqrt{2}} \kappa^{\prime} \bar{\psi}_{L 2} \mu_{R} \\
& +\frac{v_{s}}{\sqrt{2}} y \bar{\psi}_{L 2} \psi_{R 2}+M_{F} \bar{\psi}_{L} \psi_{R}
\end{aligned}
$$

where $\frac{v_{h}}{\sqrt{2}} Y_{e}=\frac{v_{h}}{\sqrt{2}} \operatorname{diag}\left(y_{e}, y_{\mu}, y_{\tau}\right)$. Diagonalizing $\mathcal{M}_{f} \mathcal{M}_{f}^{\dagger}$ and $\mathcal{M}_{f}^{\dagger} \mathcal{M}_{f}$ to get rotations for $f_{L}$ and $f_{R}$, respectively, with $m_{2}=M_{F}+\frac{v_{s}}{\sqrt{2}} y$, reveals mixing angles at the order

$$
\theta_{L}^{A} \simeq \frac{\kappa v_{h}}{\sqrt{2} M_{F}}, \quad \theta_{R}^{A} \simeq \frac{\kappa^{\prime} v_{s}}{\sqrt{2} m_{2}}
$$

for $\ell_{i L}-\psi_{L i}$ and $\mu_{R}-\psi_{R 2}$, respectively. The mixing angles (up to order of magnitude) for the different models are given in Table VI. In models B, C, E, and F, where the $\psi$ multiplets contain $Q_{F}=0$ states, left-handed rotations are introduced between the $\nu_{L}-\psi_{L}^{0}$.

In $V^{+}$, where all diagonal components of $S$ acquire a VEV, one obtains ( $i, j$ : no sum)

$$
\begin{aligned}
& \left.\frac{\partial^{2} V}{\partial h \partial h}\right|_{S, H=0}=m_{h}^{2}=-\mu^{2}+3 v_{h}^{2} \lambda+\frac{3}{2} \delta v_{s}^{2} \\
& \left.\frac{\partial^{2} V}{\partial s_{i i} \partial s_{i i}}\right|_{S, H=0}=m_{s}^{2}=-\mu_{s}^{2}+v_{s}^{2}(3 u+5 v)+\frac{1}{2} \delta v_{h}^{2} \\
& \left.\frac{\partial^{2} V}{\partial s_{i i} \partial s_{j j}}\right|_{S, H=0}=m_{s s}=2 v v_{s}^{2}-\mu_{\mathrm{det}} \frac{v_{s}}{\sqrt{2}}(i \neq j) \\
& \left.\frac{\partial^{2} V}{\partial h \partial s_{i i}}\right|_{S, H=0}=m_{s h}=\delta v_{s} v_{h} .
\end{aligned}
$$

The normalized mass eigenstates in the basis $\left(s_{11}, s_{22}\right.$, $\left.s_{33}, h\right)$ read

$$
\begin{aligned}
& h_{1}^{\prime}=\frac{1}{\sqrt{3}}\left(\cos \beta^{\prime}, \cos \beta^{\prime}, \cos \beta^{\prime}, \sqrt{3} \sin \beta^{\prime}\right), \\
& h_{2}^{\prime}=-\frac{1}{\sqrt{3}}\left(\sin \beta^{\prime}, \sin \beta^{\prime}, \sin \beta^{\prime},-\sqrt{3} \cos \beta^{\prime}\right), \\
& h_{3}^{\prime}=\frac{1}{\sqrt{2}}(-1,0,1,0), \\
& h_{4}^{\prime}=\frac{1}{\sqrt{2}}(-1,1,0,0),
\end{aligned}
$$

with corresponding eigenvalues

$$
\begin{aligned}
m_{2}^{\prime}= & \frac{1}{2}\left(m_{s}^{\prime 2}+m_{h}^{\prime 2}+2 m_{s s}\right. \\
& \left. \pm \sqrt{\left(m_{s}^{\prime 2}-m_{h}^{\prime 2}+2 m_{s s}\right)^{2}+12 m_{s h}}\right) \\
m_{3}^{\prime}= & m_{s}^{\prime 2}-m_{s s} .
\end{aligned}
$$

Thus, the mixing of the Higgs with the BSM scalars occurs only for the states $h_{1,2}^{\prime}$ and is universal. Because of the degeneracy of $m_{4}^{\prime}$, any linear combination of the states $h_{3,4}^{\prime}$ is an eigenvector, too. In the limit $\mu_{\mathrm{det}}, v \rightarrow 0$, the angle $\beta^{\prime}$ can easily be expressed as

$$
\tan 2 \beta^{\prime}=\frac{2 \sqrt{3} m_{s h}}{m_{s}^{\prime 2}-m_{h}^{\prime 2}}=\frac{2 \sqrt{3} \delta v_{h} v_{s}}{m_{s}^{\prime 2}-m_{h}^{\prime 2}}
$$

For $m_{h}^{\prime} \ll m_{s}^{\prime}$ one obtains (79). For fermion mixing, we find, similar to (D8),

$$
\theta_{L}^{A} \simeq \frac{\kappa v_{h}}{\sqrt{2} m_{2}}, \quad \theta_{R}^{A} \simeq \frac{\kappa^{\prime} v_{s}}{\sqrt{2} m_{2}}
$$

for $\ell_{i L}-\psi_{L i}$ and $\ell_{i R}-\psi_{R i}$, respectively.

\section{APPENDIX E: WEAK INTERACTIONS AFTER EWSB}

Chiral mixing between vectorlike fermions and leptons modifies their couplings with the weak bosons. Explicit rotations to the mass basis yield

$$
\begin{aligned}
\psi_{X}^{-1, \text { gauge }} & =c_{\theta_{X}} \psi_{X}^{-1}-s_{\theta_{X}} \ell_{X}, \\
\ell_{X}^{\text {gauge }} & =c_{\theta_{X}} \ell_{X}+s_{\theta_{X}} \psi_{X}^{-1}, \\
\psi_{L}^{0, \text { gauge }} & =c_{\theta_{L}^{0}} \psi_{L}^{0}-s_{\theta_{L}^{0}} \nu_{L}, \\
\nu_{L}^{\text {gauge }} & =c_{\theta_{L}^{0}} \nu_{L}+s_{\theta_{L}^{0}} \psi_{L}^{0},
\end{aligned}
$$

where $X=L, R$ and the angles $\theta$ are positive and can be found for all models in Table VI. After rotating to the mass basis, weak interactions are described by the Lagrangian 
TABLE X. Coefficients of the $W^{-}$boson interactions with fermions in the mass basis; see Eq. (E2). The nonvanishing Clebsch-Gordan coefficients are $C_{0}^{B}=-C_{-1}^{B}=\sqrt{2}, C_{0}^{C}=C_{-1}^{D}=1$, and $C_{1}^{F}=-C_{0}^{F}=\sqrt{2}$. Angles should be taken from Table VI according to the vacuum structure and the lepton flavor $\ell$.

\begin{tabular}{cccccc}
\hline \hline$\overline{f^{Q}, f^{\prime Q+1}}$ & $c_{L}^{W}$ & $c_{R}^{W}$ & $\overline{f^{Q}}, f^{\prime Q+1}$ & $c_{L}^{W}$ & $c_{R}^{W}$ \\
\hline $\bar{\ell}, \nu$ & $c_{\theta_{L}} c_{\theta_{L}^{0}}+C_{0} s_{\theta_{L}} s_{\theta_{L}^{0}}$ & 0 & $\overline{\psi^{0}}, \psi^{+1}$ & $C_{1} c_{\theta_{L}^{0}}$ & $C_{1}$ \\
$\overline{\psi^{-1}}, \psi^{0}$ & $s_{\theta_{L}} s_{\theta_{L}^{0}}+C_{0} c_{\theta_{L}} c_{\theta_{L}^{0}}$ & $C_{0} c_{\theta_{R}}$ & $\overline{\bar{\nu}, \psi^{+1}}$ & $-C_{1} s_{\theta_{L}^{0}}$ & 0 \\
$\bar{\ell}, \psi^{0}$ & $c_{\theta_{L}} s_{\theta_{L}}-C_{0} s_{\theta_{L}} c_{\theta_{L}^{0}}$ & $-C_{0} s_{\theta_{R}}$ & $\overline{\psi^{-2}}, \psi^{-1}$ & $C_{-1} c_{\theta_{L}}$ & $C_{-1} c_{\theta_{R}}$ \\
$\overline{\psi^{-1}, \nu}$ & $s_{\theta_{L}} c_{\theta_{L}^{0}}-C_{0} c_{\theta_{L}} s_{\theta_{L}^{0}}$ & 0 & $\overline{\psi^{-2}}, \ell$ & $-C_{-1} s_{\theta_{L}}$ & $-C_{-1} s_{\theta_{R}}$ \\
\hline \hline
\end{tabular}

TABLE XI. Coefficients of the $Z$ boson interactions with $Q=-1$ and $Q=0$ fermions in the mass basis [see (E2)] with $\Delta g_{V}^{\ell}=s_{\theta_{L}}^{2}\left(T_{\psi^{-1}}^{3}+\frac{1}{2}\right) \pm s_{\theta_{R}}^{2} T_{\psi^{-1}}^{3}$ and $\Delta g^{\nu}=s_{\theta_{L}^{0}}^{2}\left[T_{\psi^{0}}^{3}-\frac{1}{2}\right]$. Angles should be taken from Table VI according to the vacuum structure and the lepton flavor $\ell$.

\begin{tabular}{lcc}
\hline$\overline{\overline{f^{-1}}, f^{\prime-1}}$ & $g_{V}$ & $g_{V}$ \\
\hline $\bar{\ell}, \ell$ & $\begin{array}{c}-\frac{1}{2}+2 s_{w}^{2}+\Delta g_{V}^{\ell} \\
2\left(T_{\psi^{-1}}^{3}+s_{w}^{2}\right)-\Delta g_{V}^{\ell}\end{array}$ & $-\frac{1}{2}+\Delta g_{A}^{\ell}$ \\
$\overline{\psi^{-1}}, \psi^{-1}$ & $-\Delta g_{A}^{\ell}$ \\
$\overline{\psi^{-1}}, \ell$ & $-\frac{1}{2}\left[\sin 2 \theta_{L}\left(T_{\psi^{-1}}^{3}+\frac{1}{2}\right)+\sin 2 \theta_{R} T_{\psi^{-1}}^{3}\right]$ & $g_{A}$ \\
\hline$\overline{\overline{f^{0}}, f^{\prime 0}}$ & $g_{V}$ & $-\frac{1}{2}\left[\sin 2 \theta_{L}\left(T_{\psi^{-1}}^{3}+\frac{1}{2}\right)-\sin 2 \theta_{R} T_{\psi^{-1}}^{3}\right]$ \\
\hline $\bar{\nu}, \nu$ & $\frac{1}{2}+\Delta g^{\nu}$ & $\frac{1}{2}+\Delta g^{\nu}$ \\
$\overline{\psi^{0}}, \psi^{0}$ & $2 T_{\psi^{0}}^{3}-\Delta g^{\nu}$ & $-\Delta g^{\nu}$ \\
$\overline{\psi^{0}}, \nu$ & $-\frac{1}{2} \sin 2 \theta_{L}^{0}\left(T_{\psi^{0}}^{3}-\frac{1}{2}\right)$ & $-\frac{1}{2} \sin 2 \theta_{L}^{0}\left(T_{\psi^{0}}^{3}-\frac{1}{2}\right)$ \\
\hline \hline
\end{tabular}

$$
\begin{aligned}
\mathcal{L}_{\mathrm{W}}= & \frac{g_{2}}{2 \cos \theta_{w}} \overline{f^{Q}} \gamma^{\mu}\left(g_{V}-g_{A} \gamma^{5}\right) f^{\prime}{ }^{Q} Z_{\mu} \\
& +\frac{g_{2}}{\sqrt{2}} \overline{f^{Q}} \gamma^{\mu}\left(c_{L}^{W} P_{L}+c_{R}^{W} P_{R}\right) f^{\prime Q+1} W_{\mu}^{-}+\text {H.c. },
\end{aligned}
$$

and for all possible combinations of fermions $f, f^{\prime}$ in our models. The coefficients $c_{L, R}^{W}$ can be found in Table $\mathrm{X}$. Expressions for the couplings $g_{V, A}$ are collected in Table XI.
[1] D. J. Gross and F. Wilczek, Ultraviolet Behavior of Nonabelian Gauge Theories, Phys. Rev. Lett. 30, 1343 (1973).

[2] H. D. Politzer, Reliable Perturbative Results for Strong Interactions?, Phys. Rev. Lett. 30, 1346 (1973).

[3] D. Bailin and A. Love, Asymptotic near freedom, Nucl. Phys. B75, 159 (1974).

[4] S. Weinberg, Ultraviolet divergences in quantum theories of gravitation, in General Relativity: An Einstein Centenary Survey, edited by S. W. Hawking and W. Israel (Cambridge University Press, Cambridge, England, 1980), p. 790.

[5] D. F. Litim and F. Sannino, Asymptotic safety guaranteed, J. High Energy Phys. 12 (2014) 178.

[6] A. D. Bond and D. F. Litim, Price of Asymptotic Safety, Phys. Rev. Lett. 122, 211601 (2019).
[7] A. D. Bond and D. F. Litim, Theorems for asymptotic safety of gauge theories, Eur. Phys. J. C 77, 429 (2017); Erratum, Eur. Phys. J. C 77, 525 (2017).

[8] A. Bond and D. F. Litim, Interacting ultraviolet completions of four-dimensional gauge theories, Proc. Sci., LATTICE2016 (2017) 208.

[9] A. D. Bond, D. F. Litim, and T. Steudtner, Asymptotic safety with Majorana fermions and new large $N$ equivalences, Phys. Rev. D 101, 045006 (2020).

[10] A. D. Bond and D.F. Litim, More asymptotic safety guaranteed, Phys. Rev. D 97, 085008 (2018).

[11] A. D. Bond, G. Hiller, K. Kowalska, and D. F. Litim, Directions for model building from asymptotic safety, J. High Energy Phys. 08 (2017) 004. 
[12] D. F. Litim, M. Mojaza, and F. Sannino, Vacuum stability of asymptotically safe gauge-Yukawa theories, J. High Energy Phys. 01 (2016) 081.

[13] T. Buyukbese and D. F. Litim, Asymptotic safety of gauge theories beyond marginal interactions, Proc. Sci., LATTICE2016 (2017) 233.

[14] K. Kowalska, A. Bond, G. Hiller, and D. Litim, Towards an asymptotically safe completion of the Standard Model, Proc. Sci., EPS-HEP2017 (2017) 542.

[15] A. D. Bond and D. F. Litim, Asymptotic Safety Guaranteed in Supersymmetry, Phys. Rev. Lett. 119, 211601 (2017).

[16] A. D. Bond, D. F. Litim, G. Medina Vazquez, and T. Steudtner, UV conformal window for asymptotic safety, Phys. Rev. D 97, 036019 (2018).

[17] S. Abel and F. Sannino, Radiative symmetry breaking from interacting UV fixed points, Phys. Rev. D 96, 056028 (2017).

[18] A. Palanques-Mestre and P. Pascual, The $1 / N_{f}$ expansion of the $\gamma$ and beta functions in QED, Commun. Math. Phys. 95, 277 (1984).

[19] J. Gracey, The QCD beta function at $\mathrm{O}\left(1 / N_{f}\right)$, Phys. Lett. B 373, 178 (1996).

[20] G. M. Pelaggi, A. D. Plascencia, A. Salvio, F. Sannino, J. Smirnov, and A. Strumia, Asymptotically safe Standard Model extensions?, Phys. Rev. D 97, 095013 (2018).

[21] R. Mann, J. Meffe, F. Sannino, T. Steele, Z.-W. Wang, and C. Zhang, Asymptotically Safe Standard Model via Vectorlike Fermions, Phys. Rev. Lett. 119, 261802 (2017).

[22] K. Kowalska and E. M. Sessolo, Gauge contribution to the $1 / \mathrm{N}_{F}$ expansion of the Yukawa coupling beta function, J. High Energy Phys. 04 (2018) 027.

[23] O. Antipin, N. A. Dondi, F. Sannino, A. E. Thomsen, and Z.-W. Wang, Gauge-Yukawa theories: Beta functions at large $N_{f}$, Phys. Rev. D 98, 016003 (2018).

[24] S. Abel, E. Mølgaard, and F. Sannino, Complete asymptotically safe embedding of the Standard Model, Phys. Rev. D 99, 035030 (2019).

[25] T. Alanne, S. Blasi, and N. A. Dondi, Critical Look at $\beta$-Function Singularities at Large $N$, Phys. Rev. Lett. 123, 131602 (2019).

[26] V. Leino, T. Rindlisbacher, K. Rummukainen, F. Sannino, and K. Tuominen, Safety versus triviality on the lattice, Phys. Rev. D 101, 074508 (2020).

[27] S. P. Martin and J.D. Wells, Constraints on ultraviolet stable fixed points in supersymmetric gauge theories, Phys. Rev. D 64, 036010 (2001).

[28] H. Gies, J. Jaeckel, and C. Wetterich, Towards a renormalizable Standard Model without fundamental Higgs scalar, Phys. Rev. D 69, 105008 (2004).

[29] M. Shaposhnikov and D. Zenhausern, Quantum scale invariance, cosmological constant and hierarchy problem, Phys. Lett. B 671, 162 (2009).

[30] H. Gies, S. Rechenberger, M. M. Scherer, and L. Zambelli, An asymptotic safety scenario for gauged chiral HiggsYukawa models, Eur. Phys. J. C 73, 2652 (2013).

[31] G. M. Tavares, M. Schmaltz, and W. Skiba, Higgs mass naturalness and scale invariance in the UV, Phys. Rev. D 89, 015009 (2014).

[32] S. Abel and A. Mariotti, Novel Higgs potentials from gauge mediation of exact scale breaking, Phys. Rev. D 89, 125018 (2014).
[33] K. Intriligator and F. Sannino, Supersymmetric asymptotic safety is not guaranteed, J. High Energy Phys., 11 (2015) 023.

[34] D. Barducci, M. Fabbrichesi, C. M. Nieto, R. Percacci, and V. Skrinjar, In search of a UV completion of the standard model-378,000 models that don't work, J. High Energy Phys. 11 (2018) 057.

[35] J. McDowall and D. J. Miller, High scale boundary conditions in models with two Higgs doublets, Phys. Rev. D 100, 015018 (2019).

[36] P. Schuh, Vacuum stability of asymptotically safe two Higgs doublet models, Eur. Phys. J. C 79, 909 (2019).

[37] S. Heinemeyer, M. Mondragón, N. Tracas, and G. Zoupanos, Reduction of couplings and its application in particle physics, Phys. Rep. 814, 1 (2019).

[38] H. Gies and J. Ziebell, Asymptotically safe QED, Eur. Phys. J. C 80, 607 (2020).

[39] G. Hiller, C. Hormigos-Feliu, D. F. Litim, and T. Steudtner, Asymptotically safe extensions of the Standard Model with flavour phenomenology, in 54Th Rencontres De Moriond on Electroweak Interactions and Unified Theories (ARISF, Paris, France, 2019), pp. 415-418.

[40] G. Hiller, C. Hormigos-Feliu, D. F. Litim, and T. Steudtner, Anomalous magnetic moments from asymptotic safety, arXiv:1910.14062.

[41] Y. Nir, Probing new physics with flavor physics (and probing flavor physics with new physics), in Prospects in Theoretical Physics (PiTP) summer program on The Standard Model and Beyond IAS, Princeton, NJ, June 16-27, 2007 (Proceedings of Science, Trieste, Italy, 2007).

[42] M. E. Machacek and M. T. Vaughn, Two loop renormalization group equations in a general quantum field theory. 1. Wave function renormalization, Nucl. Phys. B222, 83 (1983).

[43] M. E. Machacek and M. T. Vaughn, Two loop renormalization group equations in a general quantum field theory. 2. Yukawa couplings, Nucl. Phys. B236, 221 (1984).

[44] M. E. Machacek and M. T. Vaughn, Two loop renormalization group equations in a general quantum field theory. 3. Scalar quartic couplings, Nucl. Phys. B249, 70 (1985).

[45] M.-X. Luo, H.-W. Wang, and Y. Xiao, Two loop renormalization group equations in general gauge field theories, Phys. Rev. D 67, 065019 (2003).

[46] I. Schienbein, F. Staub, T. Steudtner, and K. Svirina, Revisiting RGEs for general gauge theories, Nucl. Phys. B939, 1 (2019).

[47] A. G. M. Pickering, J. A. Gracey, and D. R. T. Jones, Three loop gauge beta function for the most general single gauge coupling theory, Phys. Lett. B 510, 347 (2001); Erratum, Phys. Lett. B 535, 377 (2002).

[48] L. N. Mihaila, J. Salomon, and M. Steinhauser, Renormalization constants and beta functions for the gauge couplings of the Standard Model to three-loop order, Phys. Rev. D 86, 096008 (2012).

[49] W. E. Caswell, Asymptotic Behavior of Nonabelian Gauge Theories to Two Loop Order, Phys. Rev. Lett. 33, 244 (1974).

[50] T. Banks and A. Zaks, On the phase structure of vector-like gauge theories with massless fermions, Nucl. Phys. B196, 189 (1982). 
[51] A. J. Paterson, Coleman-Weinberg symmetry breaking in the chiral $\mathrm{SU}(N) \mathrm{XSU}(N)$ linear sigma model, Nucl. Phys. B190, 188 (1981).

[52] S. Bifani, S. Descotes-Genon, A. Romero Vidal, and M.-H. Schune, Review of lepton universality tests in $B$ decays, J. Phys. G 46, 023001 (2019).

[53] M. Tanabashi et al. (Particle Data Group), Review of particle physics, Phys. Rev. D 98, 030001 (2018), and 2019 update.

[54] D. Buttazzo, G. Degrassi, P. P. Giardino, G. F. Giudice, F. Sala, A. Salvio, and A. Strumia, Investigating the nearcriticality of the Higgs boson, J. High Energy Phys. 12 (2013) 089.

[55] G. Degrassi, S. Di Vita, J. Elias-Miro, J. R. Espinosa, G. F. Giudice, G. Isidori, and A. Strumia, Higgs mass and vacuum stability in the Standard Model at NNLO, J. High Energy Phys. 08 (2012) 098.

[56] L. N. Mihaila, J. Salomon, and M. Steinhauser, Gauge Coupling Beta Functions in the Standard Model to Three Loops, Phys. Rev. Lett. 108, 151602 (2012).

[57] A. V. Bednyakov, A. F. Pikelner, and V. N. Velizhanin, Anomalous dimensions of gauge fields and gauge coupling beta-functions in the Standard Model at three loops, J. High Energy Phys. 01 (2013) 017.

[58] A. V. Bednyakov, A. F. Pikelner, and V. N. Velizhanin, Yukawa coupling beta-functions in the Standard Model at three loops, Phys. Lett. B 722, 336 (2013).

[59] A. V. Bednyakov, A. F. Pikelner, and V. N. Velizhanin, Higgs self-coupling beta-function in the Standard Model at three loops, Nucl. Phys. B875, 552 (2013).

[60] K. G. Chetyrkin and M. F. Zoller, Three-loop $\beta$-functions for top-Yukawa and the Higgs self-interaction in the Standard Model, J. High Energy Phys. 06 (2012) 033.

[61] K. G. Chetyrkin and M. F. Zoller, $\beta$-function for the Higgs self-interaction in the Standard Model at three-loop level, J. High Energy Phys. 04 (2013) 091; Erratum, J. High Energy Phys. 09 (2013) 155.

[62] C. Patrignani et al. (Particle Data Group), Review of particle physics, Chin. Phys. C 40, 100001 (2016).

[63] M. Cirelli, N. Fornengo, and A. Strumia, Minimal dark matter, Nucl. Phys. B753, 178 (2006).

[64] J. A. Evans and J. Shelton, Long-lived staus and displaced leptons at the LHC, J. High Energy Phys. 04 (2016) 056.

[65] J. F. Gunion, H. E. Haber, G. L. Kane, and S. Dawson, The Higgs Hunter's Guide, Vol. 80 (Perseus, New York City, USA, 2000).

[66] W. Altmannshofer, J. Brod, and M. Schmaltz, Experimental constraints on the coupling of the Higgs boson to electrons, J. High Energy Phys. 05 (2015) 125.

[67] D. S. M. Alves, J. Galloway, J. T. Ruderman, and J. R. Walsh, Running electroweak couplings as a probe of new physics, J. High Energy Phys. 02 (2015) 007.

[68] M. Farina, G. Panico, D. Pappadopulo, J. T. Ruderman, R. Torre, and A. Wulzer, Energy helps accuracy: Electroweak precision tests at hadron colliders, Phys. Lett. B 772, 210 (2017).

[69] F. Jegerlehner, Muon g-2 theory: The hadronic part, EPJ Web Conf. 166, 00022 (2018).
[70] M. Davier, Update of the hadronic vacuum polarisation contribution to the muon g-2, Nucl. Part. Phys. Proc. 287288, 70 (2017).

[71] S. Borsanyi et al., Leading-order hadronic vacuum polarization contribution to the muon magnetic momentfrom lattice QCD, arXiv:2002.12347.

[72] T. Aoyama et al., The anomalous magnetic moment of the muon in the Standard Model, arXiv:2006.04822.

[73] A. Crivellin, M. Hoferichter, C. A. Manzari, and M. Montull, Hadronic Vacuum Polarization: $(g-2)_{\mu}$ Versus Global Electroweak Fits, Phys. Rev. Lett. 125, 091801 (2020).

[74] A. Keshavarzi, W. J. Marciano, M. Passera, and A. Sirlin, The muon $g-2$ and $\Delta \alpha$ connection, Phys. Rev. D 102, 033002 (2020).

[75] D. Hanneke, S. Fogwell, and G. Gabrielse, New Measurement of the Electron Magnetic Moment and the Fine Structure Constant, Phys. Rev. Lett. 100, 120801 (2008).

[76] R. H. Parker, C. Yu, W. Zhong, B. Estey, and H. Müller, Measurement of the fine-structure constant as a test of the Standard Model, Science 360, 191 (2018).

[77] G. F. Giudice, P. Paradisi, and M. Passera, Testing new physics with the electron $g-2$, J. High Energy Phys. 11 (2012) 113.

[78] H. Davoudiasl and W. J. Marciano, Tale of two anomalies, Phys. Rev. D 98, 075011 (2018).

[79] J. Liu, C. E. Wagner, and X.-P. Wang, A light complex scalar for the electron and muon anomalous magnetic moments, J. High Energy Phys. 03 (2019) 008.

[80] S. Gardner and X. Yan, LIght scalars with lepton number to solve the $(g-2)_{e}$ anomaly, arXiv:1907.12571.

[81] C. Cornella, P. Paradisi, and O. Sumensari, Hunting for alps with lepton flavor violation, J. High Energy Phys. 01 (2020) 158.

[82] M. Bauer, M. Neubert, S. Renner, M. Schnubel, and A. Thamm, Axion-Like Particles, Lepton-Flavor Violation and a New Explanation of $a_{\mu}$ and $a_{e}$, Phys. Rev. Lett. 124, 211803 (2020).

[83] B. Dutta, S. Ghosh, and T. Li, Explaining $(g-2)_{\mu, e}$, KOTO anomaly and MiniBooNE excess in an extended Higgs model with sterile neutrinos, Phys. Rev. D 102, 055017 (2020).

[84] B. Dutta and Y. Mimura, Electron $g-2$ with flavor violation in MSSM, Phys. Lett. B 790, 563 (2019).

[85] M. Endo and W. Yin, Explaining electron and muon $g-2$ anomaly in SUSY without lepton-flavor mixings, J. High Energy Phys. 08 (2019) 122.

[86] M. Badziak and K. Sakurai, Explanation of electron and muon $g-2$ anomalies in the MSSM, J. High Energy Phys. 10 (2019) 024.

[87] J.-L. Yang, T.-F. Feng, and H.-B. Zhang, Electron and muon $(g-2)$ in the B-LSSM, J. Phys. G 47, 055004 (2020).

[88] A. Crivellin, M. Hoferichter, and P. Schmidt-Wellenburg, Combined explanations of (g-2)(mu,e) and implications for a large muon EDM, Phys. Rev. D 98, 113002 (2018).

[89] A. Crivellin and M. Hoferichter, Combined explanations of $(g-2)_{\mu},(g-2)_{e}$ and implications for a large muon EDM, in 54th Rencontres de Moriond on Electroweak Interactions and Unified Theories (Il Nuovo Cimento C, Bologna, Italy, 2019), pp. 29-34. 
[90] I. Bigaran and R. R. Volkas, Getting chirality right: Single scalar leptoquark solution/s to the $(g-2)_{e, \mu}$ puzzle, arXiv:2002.12544.

[91] I. Doršner, S. Fajfer, and S. Saad, $\mu \rightarrow e \gamma$ selecting scalar leptoquark solutions for the $(g-2)_{e, \mu}$ puzzles, Phys. Rev. D 102, 075007 (2020).

[92] F. J. Botella, F. Cornet-Gomez, and M. Nebot, Electron and muon $g-2$ anomalies in general flavour conserving two Higgs doublets models, Phys. Rev. D 102, 035023 (2020).

[93] S. Jana, P. K. Vishnu, and S. Saad, Resolving electron and muon $g-2$ within the 2HDM, Phys. Rev. D 101, 115037 (2020).

[94] X.-F. Han, T. Li, L. Wang, and Y. Zhang, Simple interpretations of lepton anomalies in the lepton-specific inert Two-Higgs-Doublet model, Phys. Rev. D 99, 095034 (2019).

[95] M. Abdullah, B. Dutta, S. Ghosh, and T. Li, $(g-2)_{\mu, e}$ and the ANITA anomalous events in a three-loop neutrino mass model, Phys. Rev. D 100, 115006 (2019).

[96] A. C. Hernández, Y. H. Velásquez, S. Kovalenko, H. Long, N. A. Pérez-Julve, and V. Vien, Fermion spectrum and $g-2$ anomalies in a low scale 3-3-1 model, arXiv:2002.07347.

[97] N. Haba, Y. Shimizu, and T. Yamada, Muon and electron $g-2$ and the origin of fermion mass hierarchy, Prog. Theor. Exp. Phys. 2020, 093 B05 (2020).

[98] L. Calibbi, M. López-Ibáñez, A. Melis, and O. Vives, Muon and electron $g-2$ and lepton masses in flavor models, J. High Energy Phys. 06 (2020) 087.

[99] C. Arbeláez, R. Cepedello, R. M. Fonseca, and M. Hirsch, $(g-2)$ anomalies and neutrino mass, Phys. Rev. D 102, 075005 (2020).

[100] C.-H. Chen and T. Nomura, Electron and muon $g-2$, radiative neutrino mass, and $\ell^{\prime} \rightarrow \ell \gamma$ in a $U(1)_{e-\mu}$ model, arXiv:2003.07638.

[101] C. Hati, J. Kriewald, J. Orloff, and A. Teixeira, Anomalies in ${ }^{8} \mathrm{Be}$ nuclear transitions and $(g-2)_{e, \mu}$ : Towards a minimal combined explanation, J. High Energy Phys. 07 (2020) 235.

[102] S. Jana, P. K. Vishnu, W. Rodejohann, and S. Saad, Dark matter assisted lepton anomalous magnetic moments and neutrino masses, Phys. Rev. D 102, 075003 (2020).
[103] V. Andreev et al. (ACME Collaboration), Improved limit on the electric dipole moment of the electron, Nature (London) 562, 355 (2018).

[104] G. W. Bennett et al. (Muon (g-2) Collaboration), An improved limit on the muon electric dipole moment, Phys. Rev. D 80, 052008 (2009).

[105] L. Lavoura, General formulae for $f_{1} \rightarrow f_{2} \gamma$, Eur. Phys. J. C 29, 191 (2003).

[106] G. Cavoto, A. Papa, F. Renga, E. Ripiccini, and C. Voena, The quest for $\mu \rightarrow e \gamma$ and its experimental limiting factors at future high intensity muon beams, Eur. Phys. J. C 78, 37 (2018).

[107] T. A. Chowdhury and S. Nasri, Charged lepton flavor violation in a class of radiative neutrino mass generation models, Phys. Rev. D 97, 075042 (2018).

[108] U. Bellgardt et al. (SINDRUM Collaboration), Search for the decay $\mu^{+} \rightarrow e^{+} e^{+} e^{-}$, Nucl. Phys. B299, 1 (1988).

[109] A.-K. Perrevoort (Mu3e Collaboration), Searching for lepton flavour violation with the Mu3e experiment, Proc. Sci., NuFact2017 (2017) 105 [arXiv:1802.09851].

[110] W. H. Bertl et al. (SINDRUM II Collaboration), A Search for muon to electron conversion in muonic gold, Eur. Phys. J. C 47, 337 (2006).

[111] R. Kitano, M. Koike, and Y. Okada, Detailed calculation of lepton flavor violating muon electron conversion rate for various nuclei, Phys. Rev. D 66, 096002 (2002); Erratum, Phys. Rev. D 76, 059902 (2007).

[112] R. Donghia (Mu2e Calorimeter Group), The Mu2e experiment at Fermilab, Nuovo Cimento C 40, 48 (2017).

[113] M. Benedikt et al., Future circular collider, Report No. CERN-ACC-2018-0057, 2019.

[114] F. Zimmermann, LHC/FCC-based muon colliders, in Proceedings, 9th International Particle Accelerator Conference (IPAC 2018): Vancouver, BC Canada, April 29-May 4, 2018 (JACoW Publishing, Geneva, Switzerland 2018).

[115] J. P. Leveille, The second order weak correction to (G-2) of the muon in arbitrary gauge models, Nucl. Phys. B137, 63 (1978).

[116] Y. Bai and B. A. Dobrescu, Minimal $S U(3) \times S U(3)$ symmetry breaking patterns, Phys. Rev. D 97, 055024 (2018). 